\title{
Isomonodromic Laplace transform with coalescing eigenvalues and confluence of Fuchsian singularities
}

\author{
Davide Guzzetti ${ }^{1}$
}

Received: 29 November 2020 / Revised: 15 May 2021 / Accepted: 3 June 2021 /

Published online: 17 June 2021

(c) The Author(s) 2021

\begin{abstract}
We consider a Pfaffian system expressing isomonodromy of an irregular system of Okubo type, depending on complex deformation parameters $u=\left(u_{1}, \ldots, u_{n}\right)$, which are eigenvalues of the leading matrix at the irregular singularity. At the same time, we consider a Pfaffian system of non-normalized Schlesinger-type expressing isomonodromy of a Fuchsian system, whose poles are the deformation parameters $u_{1}, \ldots, u_{n}$. The parameters vary in a polydisc containing a coalescence locus for the eigenvalues of the leading matrix of the irregular system, corresponding to confluence of the Fuchsian singularities. We construct isomonodromic selected and singular vector solutions of the Fuchsian Pfaffian system together with their isomonodromic connection coefficients, so extending a result of Balser et al. (I SIAM J Math Anal 12(5): 691-721, 1981) and Guzzetti (Funkcial Ekvac 59(3): 383-433, 2016) to the isomonodromic case, including confluence of singularities. Then, we introduce an isomonodromic Laplace transform of the selected and singular vector solutions, allowing to obtain isomonodromic fundamental solutions for the irregular system, and their Stokes matrices expressed in terms of connection coefficients. These facts, in addition to extending (Balser et al. in I SIAM J Math Anal 12(5): 691-721, 1981; Guzzetti in Funkcial Ekvac 59(3): 383-433, 2016) to the isomonodromic case (with coalescences/confluences), allow to prove by means of Laplace transform the main result of Cotti et al. (Duke Math J arXiv:1706.04808, 2017), namely the analytic theory of non-generic isomonodromic deformations of the irregular system with coalescing eigenvalues.
\end{abstract}

Keywords Non generic isomonodromy deformations - Schlesinger equations · Isomonodromic confluence of singularities - Stokes phenomenon - Coalescence of eigenvalues $\cdot$ Resonant irregular singularity $\cdot$ Stokes matrices $\cdot$ Monodromy data

\footnotetext{
$凶 \quad$ Davide Guzzetti guzzetti@sissa.it

1 SISSA, Via Bonomea, 265, 34136 Trieste, Italy
} 


\section{Contents}

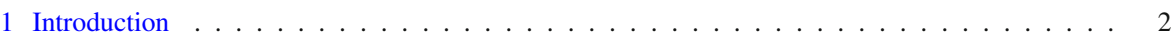

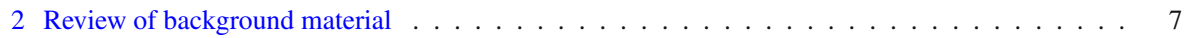

2.1 Background 1: isomonodromy deformations of (1.1) with coalescing eigenvalues . . . . 8

2.1.1 Deformations in $\mathbb{D}\left(u^{0}\right) \ldots \ldots \ldots \ldots \ldots$

2.1.2 Deformations in $\mathbb{D}\left(u^{c}\right)$ with coalescences . . . . . . . . . . . . . 12

2.2 Background 2: Laplace transform, connection coefficients and Stokes matrices . . . . . . . 16

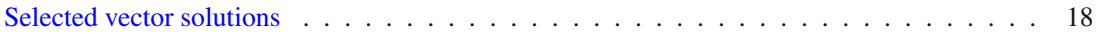

Connection coefficients . . . . . . . . . . . . . . . . . . . . . . . . . . . 19

Laplace transform and stokes matrices in terms of connection coefficients . . . . . . . . 20

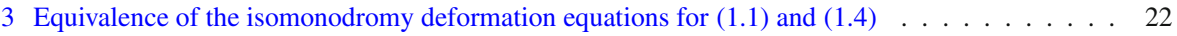

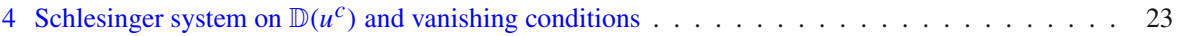

5 Selected vector solutions depending on parameters $u \in \mathbb{D}\left(u^{c}\right)$, Theorem $5.1 \ldots \ldots \ldots \ldots$

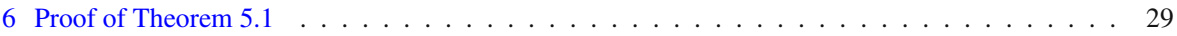

6.1 Fundamental matrix solution of the Pfaffian System . . . . . . . . . . . . . . . 30

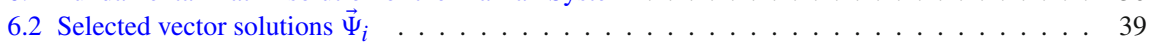

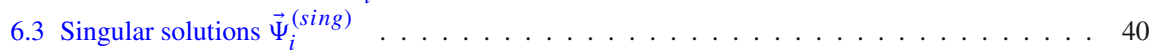

6.4 Expansions at $\lambda=u_{i}, i=1, \ldots, p_{1}$ and completion of the proof $\ldots \ldots \ldots \ldots \ldots 2$

6.5 Analogous proof for all coalescences . . . . . . . . . . . . . . . . 44

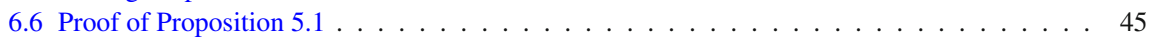

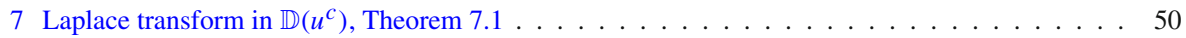

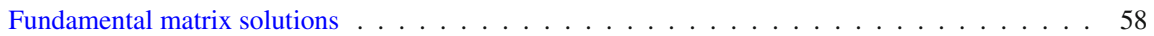

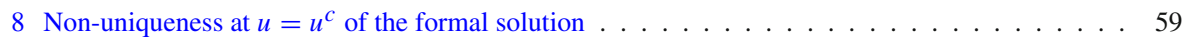

9 Appendix A. non-normalized Schlesinger system . . . . . . . . . . . . . . . . 62

10 Appendix B. Proof of Proposition $3.1 \ldots \ldots \ldots \ldots$. . . . . . . . . . . . . . . . 64

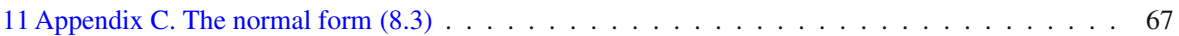

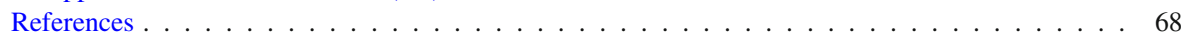

\section{Introduction}

In this paper, I answer a question asked when I presented the results of [13] and the related paper [25]. Paper [13] deals with the extension of the theory of isomonodromic deformations of the differential system (1.1), in the presence of a coalescence phenomenon involving the eigenvalues of the leading matrix $\Lambda$. These eigenvalues are the deformation parameters. The question is if we can obtain some results of [13] in terms of the Laplace transform relating system (1.1) to a Fuchsian one, such as system (1.4). The latter has simple poles at the eigenvalues of $\Lambda$, so that the coalescence of the eigenvalues will correspond to the confluence of the Fuchsian singularities. So the question is if combining integrable deformations of Fuchsian systems, confluence of singularities and Laplace transform, we can obtain the results of [13]. The positive answer is Theorem 7.1 of this paper. In order to achieve it, we extend to the case depending on deformation parameters, including their coalescence, one main result of $[4,23]$ concerning the existence of selected and singular vector solutions of a Pfaffian Fuchsian system associated with (1.4) (see the system (5.3)), and their connection coefficients, which will be isomonodromic. This will be obtained in Theorem 5.1 and Proposition 5.1.

In [13], the isomonodromy deformation theory of an $n$-dimensional differential system with Fuchsian singularity at $z=0$ and singularity of the second kind at 
$z=\infty$ of Poincaré rank 1

$$
\frac{\mathrm{d} Y}{\mathrm{~d} z}=\left(\Lambda(u)+\frac{A(u)}{z}\right) Y, \quad \Lambda(u)=\operatorname{diag}\left(u_{1}, \ldots, u_{n}\right),
$$

has been considered ${ }^{1}$. The deformation parameters $u=\left(u_{1}, \ldots, u_{n}\right)$ vary in a polydisc where the matrix $A(u)$ is holomorphic. One of the main results of [13] is the extension of the theory of isomonodromic deformations of (1.1) to the non-generic case when $\Lambda$ has coalescing eigenvalues but remains diagonalizable. This means that the polydisc contains a locus of coalescence points such that $u_{i}=u_{j}$ for some $1 \leq i \neq j \leq n$. In this case, $z=\infty$ is sometimes called resonant irregular singularity. On a sufficiently small domain in the polydisc, the well-known theory of isomonodromy deformations applies and allows to define constant monodromy data. Theorem 1.1 and corollary 1.1 of [13] say that these data are well defined and constant on the whole polydisc, including the coalescence locus, if the entries of $A(u)$ satisfy the vanishing conditions

$(A(u))_{i j} \rightarrow 0$ when $u$ tends to a coalescence point such that $u_{i}-u_{j} \rightarrow 0$ at this point.

More precisely, if conditions (1.2) are satisfied, the following results (reviewed in Theorem 2.2 of Sect. 2.1) hold.

(I) Fundamental matrix solutions in Levelt form at $z=0$ and solutions with prescribed "canonical" asymptotic behaviour in Stokes sectors at $z=\infty$ are holomorphic of $u$ in the polydisc. Also the coefficients of the formal solution determining the asymptotics at $\infty$ are holomorphic.

(II) Essential monodromy data, such as Stokes matrices, the central connection matrix, the formal monodromy exponent at infinity and the Levelt exponents at $z=0$ are well defined and constant on the whole polydisc, including coalescence points. The Stokes matrices (labelled by $v \in \mathbb{Z}$ ) satisfy the vanishing conditions

$$
\left(\mathbb{S}_{\mathcal{v}}\right)_{i j}=\left(\mathbb{S}_{\nu}\right)_{j i}=0, i \neq j \text {, if there is a coalescence point such that } u_{i}=u_{j}
$$

(III) The constant essential monodromy data can be computed from the system "frozen" at a fixed coalescence point. In particular, if the constant diagonal entries of $A$ are partly non-resonant (see Corollary 2.1), then there is no ambiguity in this computation, being the formal solution unique.

The results above have been established in [13] by direct analysis of system (1.1), of its Stokes phenomenon and its isomonodromic deformations.

Remark 1.1 If $A(u)$ is holomorphic on the polydisc and (1.1) is an isomonodromic family on the polydisc minus the coalescence locus (in the sense of integrability of an associated Pfaffian system (2.14) introduced later), then (1.2) are automatically satisfied and Theorem 1.1 of [13] holds. This is not mentioned in [13]. I thank the referee for this observation. More details are in Remark 2.1.

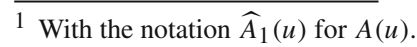


For future use, we denote by $\lambda_{1}^{\prime}, \ldots, \lambda_{n}^{\prime}$ the diagonal entries of $A(u)$, and

$$
B:=\operatorname{diag}(A(u))=\operatorname{diag}\left(\lambda_{1}^{\prime}, \ldots, \lambda_{n}^{\prime}\right) .
$$

We will see that these $\lambda_{k}^{\prime}$ are constant in the isomonodromic case.

From another perspective, if $u$ is fixed and $u_{i} \neq u_{j}$ for $i \neq j$, namely for a system (1.1) not depending on parameters with pairwise distinct eigenvalues of $\Lambda$, it is well known that columns of fundamental matrix solutions with prescribed asymptotics in Stokes sectors at $z=\infty$ can be obtained by Laplace-type integrals of certain selected column-vector solutions of an $n$-dimensional Fuchsian system of the type

$$
\frac{\mathrm{d} \Psi}{\mathrm{d} \lambda}=\sum_{k=1}^{n} \frac{B_{k}}{\lambda-u_{k}} \Psi, \quad B_{k}:=-E_{k}(A+I) .
$$

Here, $E_{k}$ is the elementary matrix whose entries are zero, except for $\left(E_{k}\right)_{k k}=1$. These facts are studied in the seminal paper [4] in the generic case of non-integer diagonal entries $\lambda_{k}^{\prime}$ of $A$. The results of [4] have been extended in [23] to the general case, when the entries $\lambda_{k}^{\prime}$ take any complex value.

The purpose of the present paper is to introduce an isomonodromic Laplace transform relating (1.1) to an isomonodromic Fuchsian system

$$
\frac{\mathrm{d} \Psi}{\mathrm{d} \lambda}=\sum_{k=1}^{n} \frac{B_{k}(u)}{\lambda-u_{k}} \Psi, \quad B_{k}:=-E_{k}(A(u)+I) .
$$

when $u_{1}, \ldots, u_{n}$ vary in a polydisc containing a locus of coalescence points. More precisely, the Laplace transform will relate solutions of the integrable Pfaffian systems (2.14) and (5.3) introduced later, associated with (1.1) and (1.4), respectively. The two main goals will be:

- Theorem 5.1, which characterizes selected vector solutions and singular vector solutions of (1.4) and (5.3), so extending the results of [4] and [23] to the case depending on isomonodromic deformation parameters, including coalescing Fuchsian singularities $u_{1}, \ldots, u_{n}$.

- Theorem 7.1, in which the Laplace transform of the vector solutions of Theorem 5.1 allows to obtain the main results (I), (II) and (III) of [13] in the presence of coalescing eigenvalues $u_{1}, \ldots, u_{n}$ of $\Lambda(u)$.

In details.

- In Proposition 3.1 we establish the equivalence between the "strong" isomonodromic deformations (non-normalized Schlesinger deformations) of (1.4) and the "strong" isomonodromic deformations of (1.1).

- Then, we study isomonodromy deformations of (1.4) when $u$ varies in a polydisc containing a coalescence locus. Theorem 5.1, provides selected and singular vector solutions, which are the isomonodromic analogue of solutions introduced in [4,23], respectively, denoted by $\vec{\Psi}_{k}(\lambda, u \mid v)$ and $\vec{\Psi}_{k}^{(\text {sing })}(\lambda, u \mid v), k=1, \ldots, n$, the latter 
being singular at $\lambda=u_{k}$. As will be explained later, $v \in \mathbb{Z}$ labels the directions of branch cuts in the punctured $\lambda$-plane at the poles $u_{1}, \ldots, u_{n}$. These solutions allow to introduce connection coefficients $c_{j k}^{(v)}$, defined by

$$
\vec{\Psi}_{k}(\lambda, u \mid v)=\vec{\Psi}_{j}^{(\text {sing })}(\lambda, u \mid v) c_{j k}^{(v)}+\text { holomorphic part at } \lambda=u_{j}, \quad \forall j \neq k .
$$

The above is the deformation parameters dependent analogue of the definition of connection coefficients in [23].

- In Proposition 5.1, we prove that the $c_{j k}^{(v)}$ are isomonodromic connection coefficients, namely independent of $u$. When there is a coalescence $u_{j}=u_{k}$ in the polydisc, they satisfy

$$
c_{j k}^{(v)}=0, \quad j \neq k
$$

- In Theorem 7.1, the Laplace transform of the vectors $\vec{\Psi}_{k}(\lambda, u \mid v)$ or $\vec{\Psi}_{k}^{(\operatorname{sing})}(\lambda, u \mid v)$ yields the columns of the isomonodromic fundamental matrix solutions $Y_{v}(z, u)$ of (1.1), labelled by $v \in \mathbb{Z}$, uniquely determined by a prescribed asymptotic behaviour in certain $u$-independent sectors $\widehat{\mathcal{S}}_{v}$, of central opening angle greater than $\pi$. The analytic properties for the matrices $Y_{v}(z, u)$ will be proved, so reobtaining the result (I) above. In order to describe the Stokes phenomenon, only three solutions $Y_{v}(z, u), Y_{v+\mu}(z, u)$ and $Y_{v+2 \mu}(z, u)$ suffice. The labelling will be explained later. The Stokes matrices $\mathbb{S}_{v+k \mu}, k=0,1$, defined by a relation $Y_{v+(k+1) \mu}=Y_{\nu+k \mu} \mathbb{S}_{\nu+k \mu}$, will be expressed in terms of the coefficients $c_{j k}^{(v)}$ in formula (7.9). This extends to the isomonodromic case, including coalescences, an analogous expression appearing in $[4,23]$ and implies the results in (II) above.

- In Sect. 8, we re-obtain the result (III), that system (1.1), "frozen" by fixing $u$ equal to the most coalescence point $u^{c}$ in the polydisc (see Sect. 2.1 for $u^{c}$ ), admits a unique formal solution if and only if the (constant) diagonal entries $\lambda_{j}^{\prime}$ of $A$ satisfy $\lambda_{i}^{\prime}-\lambda_{j}^{\prime} \notin \mathbb{Z} \backslash\{0\}$ for every $i \neq j$ such that $u_{i}^{c}=u_{j}^{c}$. In this case we prove that the selected vector solutions of the Fuchsian system (1.4) at $u=u^{c}$, needed to perform the Laplace transforms, are uniquely determined. On the other hand, if some $\lambda_{i}^{\prime}-\lambda_{j}^{\prime} \in \mathbb{Z} \backslash\{0\}$ corresponding to $u_{i}^{c}=u_{j}^{c}$, then there is a family of solutions of the Fuchsian system (1.4) at a coalescence point, depending on a finite number of parameters: this facts is responsible, through the Laplace transform, of the existence of a family of formal solutions at the coalescence point.

In $[19,20]$, B. Dubrovin related system (1.1) to an isomonodromic system of type (1.4), in the specific case when they, respectively, yield the flat sections of the deformed connection of a semisimple Dubrovin-Frobenius manifold and the flat sections of the intersection form (extended Gauss-Manin system). In $[19,20]$, the solutions of (1.1) are expressed by Laplace transform of the isomonodromic system (1.4), but the eigenvalues $u_{1}, \ldots, u_{n}$ are assumed to be pairwise distinct in a sufficiently small domain (analogous to the polydisc $\mathbb{D}\left(u^{0}\right)$ to be introduced later). Moreover, $A$ is skew-symmetric, so its diagonal elements are zero ( $A$ is denoted by $V$ and $\Lambda$ by $U$ in $[19,20])$. By a Coxeter-type identity, the entries of the monodromy matrices for 
the selected solutions of (1.4) (which are part of the monodromy of the DubrovinFrobenius manifold) are expressed in terms of the entries of the Stokes matrices. See also $[21,61]$.

In proposition 2.5.1 of [22], the authors prove (I) when system (1.1) is associated with a Dubrovin-Frobenius manifold with semisimple coalescence points, and $A$ is skew-symmetric (in [22] the irregular singularity is at $z=0$ ). Their proof contains the core idea that the analytic properties of a solution $Y(z, u)$ in (I) are obtainable, by a Laplace transform, from the analytic properties of a fundamental matrix solution $\Psi(\lambda, u)$ of the Fuchsian Pfaffian system associated with (1.4) (see their Lemma 2.5.3). The latter is a particular case of the Pfaffian systems studied in [63]. On the other hand, the analysis of selected and singular vector solutions of the Fuchsian Pfaffian system, required in our paper to cover all possible cases (all possible $A$ ), is not necessary in [22], due to the skew-symmetry of $A$, and the specific form of their Pfaffian system (see their equation (2.5.2); their discussion is equivalent our case $\lambda_{j}^{\prime}=-1$ for all $j=1, \ldots, n)$. Moreover, points (II) and (III) are not discussed in [22] by means of the Laplace transform.

In the present paper, by an isomonodromic Laplace transform, we prove (I), (II) and (III), and at the same time we generalize the results of $[4,23]$ to the isomonodromic case with coalescences, with no assumptions on the eigenvalues and the diagonal entries of $A$. This analytic construction, to the best of our knowledge, cannot be found in the literature.

The approach of the present paper may also be used to extend the results of [19, 20] described above, relating the deformed flat connection and the intersection form, namely Stokes matrices and monodromy group of the Dubrovin-Frobenius manifold, in case of semisimple coalescent Frobenius structures studied in [10,14,15,17].

For further comments and reference on the use of the Laplace transform, the confluence of singularities and related topics, see the introduction of [23] and [9,29,3639,44,49,56-59].

Stickily related to ours are the important results of [52]. In [13] (and in the present paper by Laplace transform), we have answered the question if the integrable deformation (2.14) of system (1.1) extends from a polydisc (or a small open set) not containing coalescence points to a wider domain intersecting (a stratum of) the coalescence locus, and we have characterized the monodromy data. The converse question is answered in [52], namely if an integrable deformation (2.14) of $\left(\Lambda\left(u^{c}\right)+A\left(u^{c}\right) / z\right) \mathrm{d} z$ exists and is unique, having formal normal form $d(z \Lambda(u))+B / z \mathrm{~d} z$, where $B$ is the diagonal of $A\left(u^{c}\right)$. More broadly, the question of [52] is the existence and uniqueness of integrable deformations of meromorphic connections on $\mathbb{P}^{1}$ with irregular singularity, when a prefixed restriction is given at a single point $t_{o}$ in the space of deformation parameters $T$, allowed to be a degenerate point, namely a coalescence point in our case (in [52], deformation parameters are called $t \in T$ ). One asks if a connection $\omega\left(z, t_{0}\right)$ given at $t_{o} \in T$ can be deformed to $\omega(z, t)$, and if this deformation is unique. ${ }^{2}$ Concerning uniqueness, for a fixed normal form $\omega_{0}(z, t)$, the problem is to classify isomorphism pairs $(\omega, G)$ consisting of an integrable connection $\omega(z, t)$ (with poles

2 The notation $\omega$ and $G$ is not taken from [52]. 
in $T \times\{z=0\}$, being $z=0$ used in [52], while $z=\infty$ is used in our works) and a formal gauge transformation $G(z, t)$ (formal in $z$ but holomorphic in $t$ ), transforming $\omega(z, t)$ to $\omega_{0}(z, t)$. In a general context, a uniqueness theorem is proved in [60]: two pairs are isomorphic (meaning that the composition of a gauge of one pair with the inverse gauge of the other pair is convergent w.r.t. $z$ ) if and only if their restriction to any specific value $t_{o}$ are isomorphic. Thus, the $t$-extension of a pair in a neighbourhood of $t_{0}$ is unique up to isomorphism. The proof in [60] makes use of the results of Kedlaya [34,35] and Mochizuki [45-48], which allows to blow up $T \times\{0\}$, and of the higher-dimensional asymptotic analysis in poli-sectors for the formal gauge transformations, that is Majima's asymptotic analysis [40] for Pfaffian systems with irregular singularities. In [52], the uniqueness result is proved for a restricted class of integrable connections, in which our (2.14) is contained (with irregular singularity at $z=0$ instead of $\infty$ ). So, given a block-diagonal normal form $\omega_{0}(z, t)$ and a pair consisting of $\omega\left(t_{o}, z\right)$ and a formal gauge $G\left(t_{o}, z\right)$, it is proved that the pair can be deformed (existence) in a unique way (uniqueness) to $\omega(z, t), G(z, t)$, such that $G[\omega]=\omega_{0}$. The strategy is to use a sequence of Kedlaya-Mochizuki blow-ups to obtain a good normal form (see also [50,51]). Then, Majima results on asymptotic analysis can be used and adapted. In our specific case, theorem 4.9 of [52] means the existence and uniqueness of the integrable deformation (2.14) of $\left(\Lambda\left(u^{c}\right)+A\left(u^{c}\right) / z\right) \mathrm{d} z$, formally equivalent to $d(z \Lambda(u))+B / z \mathrm{~d} z$. These facts generalize results of Malgrange $[41,42]$ for irregular singularities to the case of coalescence points.

Theorem 4.9, obtained in [52] in geometric terms, has been successively proved in [11] by analytic methods. In [11], the integrable deformation is obtained from prefixed monodromy data at a coalescence point, using the analytic $L^{p}$ theory a RiemannHilber boundary value problems. Both authors of [52] and [11] apply their results to semisimple Dubrovin-Frobenius manifolds. In particular, [11] proves that any formal semisimple Frobenius manifold is the completion of a pointed germ of an analytic Dubrovin-Frobenius manifold. The result is extended to $F$-manifolds in the recent work [12].

A geometric formulation of the Laplace transform we have used here, together with a synthetic proof of part of Theorem 1.1 of [13], is the object of the recent work [53].

\section{Review of background material}

This section contains known material to motivate and understand our paper. For $X$ a topological space, we denote by $\mathcal{R}(X)$ its universal covering. For $\alpha<\beta \in \mathbb{R}$, a sector is written as

$$
S(\alpha, \beta):=\{z \in \mathcal{R}(\mathbb{C} \backslash\{0\}) \text { such that } \alpha<\arg z<\beta\} \text {. }
$$




\subsection{Background 1: isomonodromy deformations of (1.1) with coalescing eigenvalues}

We review some results of $[13,25]$ (see also [16,24,26]). Consider a differential system (1.1) with an $n \times n$ with matrix coefficient $A(u)$ holomorphic in a polydisc

$$
\mathbb{D}\left(u^{c}\right):=\left\{u \in \mathbb{C}^{n} \text { such that } \max _{1 \leq j \leq n}\left|u_{j}-u_{j}^{c}\right| \leq \epsilon_{0}\right\}, \quad \epsilon_{0}>0,
$$

centered at a coalescence point $u^{c}=\left(u_{1}^{c}, \ldots, u_{n}^{c}\right)$, so called because

$$
u_{i}^{c}=u_{j}^{c} \quad \text { for some } i \neq j \text {. }
$$

The eigenvalues of $\Lambda(u)$ coalesce at $u^{c}$ and also along the following coalescence locus

$$
\Delta:=\mathbb{D}\left(u^{c}\right) \cap\left(\bigcup_{i \neq j}\left\{u_{i}-u_{j}=0\right\}\right),
$$

We assume that $\mathbb{D}\left(u^{c}\right)$ is sufficiently small so that $u^{c}$ is the most coalescent point. Namely, if $u_{j}^{c} \neq u_{k}^{c}$ for some $j \neq k$, then $u_{j} \neq u_{k}$ for all $u \in \mathbb{D}\left(u^{c}\right)$. A more precise characterization of the radius $\epsilon_{0}$ of the polydisc will be given in Sect. 5. For $u^{0} \in \mathbb{D}\left(u^{c}\right) \backslash \Delta$, let

$$
\mathbb{D}\left(u^{0}\right) \subset\left(\mathbb{D}\left(u^{c}\right) \backslash \Delta\right)
$$

be a (smaller) polydisc centered at $u^{0}$, not containing coalescence points.

\subsubsection{Deformations in $\mathbb{D}\left(u^{0}\right)$}

If $\mathbb{D}\left(u^{0}\right)$ is sufficiently small, the isomonodromic theory of Jimbo, Miwa and Ueno [33] assures that the essential monodromy data of (1.1) (see Definition 2.1) are constant over $\mathbb{D}\left(u^{0}\right)$ and can be computed fixing $u=u^{0}$.

In order to give fundamental solutions with "canonical" form at $z=\infty$, in $\mathcal{R}(\mathbb{C} \backslash\{0\})$ we introduce the Stokes rays of $\Lambda\left(u^{0}\right)$, defined by

$$
\Re\left(\left(u_{j}^{0}-u_{k}^{0}\right) z\right)=0, \quad \Im\left(\left(u_{j}^{0}-u_{k}^{0}\right) z\right)<0, \quad 1 \leq j \neq k \leq n .
$$

Let

$$
\arg z=\tau^{(0)}
$$

be a direction which does not coincide with any of the Stokes rays of $\Lambda\left(u^{0}\right)$, called admissible at $u^{0}$. Each sector of amplitude $\pi$, whose boundaries are not Stokes rays of $\Lambda\left(u^{0}\right)$, contains a certain number $\mu^{(0)} \geq 1$ of Stokes rays of $\Lambda\left(u^{0}\right)$, with angular directions

$$
\arg z=\tau_{0}, \tau_{1}, \ldots, \tau_{\mu^{(0)}-1}, \quad \text { with } \quad \tau_{0},<\tau_{1}<\cdots<\tau_{\mu^{(0)}-1}
$$


Fig. 1 Successive sectors $\mathcal{S}_{v}\left(\mathbb{D}\left(u^{0}\right)\right)$ and $\mathcal{S}_{v+\mu}\left(\mathbb{D}\left(u^{0}\right)\right)$. Their intersection (in the right part of the figure) does not contain Stokes rays. It contains the admissible direction $\arg z=\tau^{(0)}$

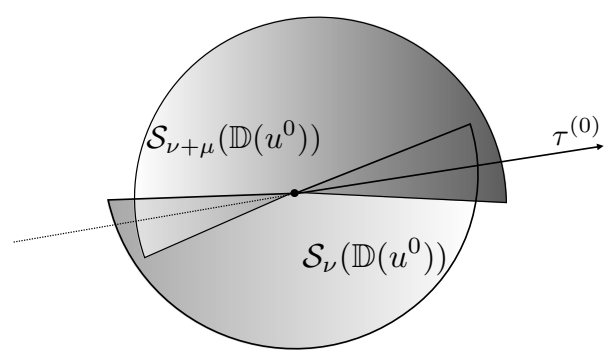

that we decide to label from 0 to $\mu^{(0)}-1$. They are basic rays, since they generate all the Stokes rays in $\mathcal{R}(\mathbb{C} \backslash\{0\})$ associated with $\Lambda\left(u^{0}\right)$ by the formula

$$
\arg z=\tau_{v}:=\tau_{v_{0}}+k \pi, \quad v_{0} \in\left\{0, \ldots, \mu^{(0)}-1\right\}, \quad v=v_{0}+k \mu^{(0)}, \quad k \in \mathbb{Z} .
$$

The choice to label a specific Stokes ray with 0 , as $\tau_{0}$ above, is arbitrary, and it induces the labelling $v \in \mathbb{Z}$ for all other rays. Suppose the labelling has been chosen. Then, for some $v \in \mathbb{Z}$, we have

$$
\tau_{v}<\tau^{(0)}<\tau_{v+1} .
$$

Equivalently, given $\tau^{(0)}$, one can choose a $v$ and decide to call $\tau_{v}$ and $\tau_{v+1}$ the Stokes rays satisfying (2.3). This induces the labelling of all other rays (notice that $\mu^{(0)}$ is not a choice!).

Similarly, we consider the Stokes rays $\Re\left(\left(u_{j}-u_{k}\right) z\right)=0, \Im\left(\left(u_{j}-u_{k}\right) z\right)<0$ of $\Lambda(u)$. If $\mathbb{D}\left(u^{0}\right)$ is sufficiently small, when $u$ varies the Stokes rays of $\Lambda(u)$ rotate without crossing $\arg z=\tau^{(0)} \bmod \pi$. For $k \in \mathbb{Z}$, we take the sector $S\left(\tau^{(0)}+(k-\right.$ 1) $\left.\pi, \tau^{(0)}+k \pi\right)$ and extend it in angular amplitude up to the nearest Stokes rays of $\Lambda(u)$ outside. The resulting (open) sector will be denoted by $\mathcal{S}_{\nu+k \mu^{(0)}}(u)$, and we define

$$
\mathcal{S}_{\nu+k \mu^{(0)}}\left(\mathbb{D}\left(u^{0}\right)\right):=\bigcap_{u \in \mathbb{D}\left(u^{0}\right)} \mathcal{S}_{\nu+k \mu^{(0)}}(u) .
$$

The reason for the labelling is that $S\left(\tau^{(0)}+(k-1) \pi, \tau^{(0)}+k \pi\right) \subset S\left(\tau_{\nu+k \mu^{(0)}}-\right.$ $\pi, \tau_{\nu+k \mu^{(0)}+1}$ ) and consequently

$\mathcal{S}_{\nu+k \mu^{(0)}}\left(\mathbb{D}\left(u^{0}\right)\right) \subset S\left(\tau_{\nu+k \mu^{(0)}}-\pi, \tau_{\nu+k \mu^{(0)}+1}\right) \equiv S\left(\tau_{\left[\nu+k \mu^{(0)}\right]-\mu^{(0)}}, \tau_{\left[v+k \mu^{(0)}\right]+1}\right)$

By construction, $\mathcal{S}_{v}\left(\mathbb{D}\left(u^{0}\right)\right)$ has central angular opening greater than $\pi$. See Fig. 1 .

Proposition 2.1 (Sibuya [30,54,55]; see also [13,25,33]). Let $\mathbb{D}\left(u^{0}\right)$, not containing coalescence points, be sufficiently small so that the Stokes rays of $\Lambda(u)$ do not cross ${ }^{3}$ the admissible rays $\arg z=\tau^{(0)}+h \pi, h \in \mathbb{Z}$, as $u$ varies in $\mathbb{D}\left(u^{0}\right)$. System (1.1) has

\footnotetext{
3 As $u$ varies, $\arg z=\tau^{(0)}+h_{0} \pi$, for a $h_{0} \in \mathbb{Z}$, is not crossed by a Stokes ray if and only if $\arg z=\tau^{(0)}+h \pi$ is not crossed $\forall h \in \mathbb{Z}$.
} 
$a$ unique formal solution

$$
Y_{F}(z, u)=F(z, u) z^{B(u)} \exp \{z \Lambda(u)\}, \quad B(u):=\operatorname{diag}\left(A_{11}(u), \ldots, A_{n n}(u)\right),
$$

where

$$
F(z, u)=I+\sum_{k=1}^{\infty} F_{k}(u) z^{-k}
$$

is a formal series, with holomorphic matrix coefficients $F_{k}(u)$. For every $v \in \mathbb{Z}$, there exist unique fundamental matrix solutions

$$
Y_{v}(z, u)=\widehat{Y}_{\nu}(z, u) z^{B(u)} \exp \{z \Lambda(u)\}
$$

of (1.1), holomorphic on $\mathcal{R}\left(\mathbb{C} \backslash\{0\} \times \mathbb{D}\left(u^{0}\right)\right) \equiv \mathcal{R}(\mathbb{C} \backslash\{0\}) \times \mathbb{D}\left(u^{0}\right)$, such that uniformly in $u \in \mathbb{D}\left(u^{0}\right)$ the following asymptotic behaviour holds

$$
\widehat{Y}_{v}(z, u) \sim F(z, u) \quad \text { for } z \rightarrow \infty \text { in } \mathcal{S}_{v}\left(\mathbb{D}\left(u^{0}\right)\right)
$$

The coefficients $F_{k}$ are computed recursively [13,62]

$$
\begin{aligned}
& \left(F_{1}\right)_{i j}=\frac{A_{i j}}{u_{j}-u_{i}}, i \neq j, \quad\left(F_{1}\right)_{i i}=-\sum_{j \neq i} A_{i j}\left(F_{1}\right)_{j i}, \\
& \left(F_{k}\right)_{i j}=\frac{1}{u_{j}-u_{i}}\left\{\left(A_{i i}-A_{j j}+k-1\right)\left(F_{k-1}\right)_{i j}+\sum_{p \neq i} A_{i p}\left(F_{k-1}\right)_{p j}\right\}, i \neq j ; \\
& k\left(F_{k}\right)_{i i}=-\sum_{j \neq i} A_{i j}\left(F_{k}\right)_{j i} .
\end{aligned}
$$

Holomorphic Stokes matrices $\mathbb{S}_{v}(u), v \in \mathbb{Z}$, are the connection matrices defined by

$$
Y_{\nu+\mu^{(0)}}(z, u)=Y_{\nu}(z, u) \mathbb{S}_{\nu}(u), \quad z \in \mathcal{S}_{\nu}\left(\mathbb{D}\left(u^{0}\right)\right) \cap \mathcal{S}_{v+\mu^{(0)}}\left(\mathbb{D}\left(u^{0}\right)\right)
$$

Notice that $\mathcal{S}_{v}\left(\mathbb{D}\left(u^{0}\right)\right) \cap \mathcal{S}_{v+\mu^{(0)}}\left(\mathbb{D}\left(u^{0}\right)\right)$ does not contain Stokes rays of $\Lambda(u)$, for every $u \in \mathbb{D}\left(u^{0}\right)$.

At every fixed $u \in \mathbb{D}\left(u^{0}\right)$, system (1.1) admits a fundamental matrix solution in Levelt form

$$
Y^{(0)}(z, u)=G^{(0)}(u)\left(I+\sum_{j=1}^{\infty} \Psi_{j}(u) z^{j}\right) z^{D} z^{L},
$$

where the series is convergent absolutely in every ball $|z|<N$, for every $N>0$. Here, $D$ is diagonal with integer entries (called valuations), $L$ has eigenvalues with real part lying in $[0,1)$, and $D+\lim _{z \rightarrow 0} z^{D} L z^{-D}$ is a Jordan form of $A$. A central 
connection matrix $C_{v}(u)$ is defined by

$$
Y_{v}(z, u)=Y^{(0)}(z, u) C_{v}(u)
$$

A pair of Stokes matrices $\mathbb{S}_{\nu}, \mathbb{S}_{v+\mu^{(0)}}$, together with $B, C_{v}$ and $L$ are sufficient to calculate all the other $\mathbb{S}_{v^{\prime}}$ and $C_{v^{\prime}}$, for all $v^{\prime} \in \mathbb{Z}$ (see $[1,13]$ ). The monodromy matrices at $z=0$ are

$$
M:=e^{2 \pi i L} \quad \text { and } \quad e^{2 \pi i B}\left(\mathbb{S}_{\nu} \mathbb{S}_{\nu+\mu^{(0)}}\right)^{-1}=C_{\nu}^{-1} M C_{\nu}
$$

for $Y^{(0)}$ and $Y_{v}$, respectively. Hence, it makes sense to define strong isomonodromy deformations, as follows.

Definition 2.1 Fixed a $v \in \mathbb{Z}$, we call essential monodromy data the matrices

$$
\mathbb{S}_{\nu}, \quad \mathbb{S}_{\nu+\mu^{(0)}}, \quad B, \quad C_{\nu}, \quad L, \quad D .
$$

The deformation $u$ is strongly isomonodromic on $\mathbb{D}\left(u^{0}\right)$, if the essential monodromy data are constant on $\mathbb{D}\left(u^{0}\right)$.

We introduced the terminology strong in [25], to mean that all the essential monodromy data are constant, contrary to the case of weak isomonodromic deformations, which only preserve monodromy matrices of a certain fundamental matrix solution. For a deformation to be weakly isomonodromic it is necessary and sufficient that (1.1) is the $z$-component of a certain Pfaffian system $d Y=\omega(z, u) Y$, Frobenius integrable (i.e. $\mathrm{d} \omega=\omega \wedge \omega$ ). If $\omega$ is of very specific form, the deformation becomes strongly isomonodromic, according to the following

Theorem 2.1 System (1.1) is strongly isomonodromic in $\mathbb{D}\left(u^{0}\right)$ if and only $Y_{v}(z, u)$, for every $v$, and $Y^{(0)}(z, u)$, satisfy the Frobenius integrable Pfaffian system

$$
d Y=\omega(z, u) Y, \quad \omega(z, u)=\left(\Lambda(u)+\frac{A(u)}{z}\right) \mathrm{d} z+\sum_{k=1}^{n} \omega_{k}(z, u) \mathrm{d} u_{k}
$$

with the matrix coefficients (here $F_{1}$ is in (2.8))

$$
\omega_{k}(z, u)=z E_{k}+\omega_{k}(u), \quad \omega_{k}(u)=\left[F_{1}(u), E_{k}\right] .
$$

Equivalently, (1.1) is strongly isomonodromic if and only if ${ }^{4}$ A satisfies

$$
d A=\sum_{j=1}^{n}\left[\omega_{k}(u), A\right] \mathrm{d} u_{k} .
$$

\footnotetext{
${ }^{4}$ Conditions (2.15) and (2.16) imply Frobenius integrability of (2.14), so that the deformation is strongly isomonodromic. Conversely, given (2.14) with $\omega_{k}(z, u)$ holomorphic in $\mathbb{C} \times \mathbb{D}\left(u^{0}\right)$, with $z=\infty$ at most a pole, then the integrability $\mathrm{d} \omega(z, u)=\omega(z, u) \wedge \omega(z, u)$, which is necessary condition for isomonodromicity, implies that $\omega_{k}(z, u)=z E_{k}+\omega_{k}(0, u)$ and (2.16). Computations give that $\omega_{k}(0, u)=$ $\left[F_{1}(u), E_{k}\right]+\mathcal{D}_{k}(u)$, where $\mathcal{D}_{k}(u)$ is an arbitrary diagonal holomorphic matrix. Imposing that $Y^{(0)}(z, u)$ and all the $Y_{v}(z, u)$ satisfy $(2.14)$, then $\mathcal{D}_{k}(u)=0$ and $\omega_{k}(0, u)=\left[F_{1}(u), E_{k}\right]$.
} 
If the deformation is strongly isomonodromic, then $Y^{(0)}(z, u)$ in (2.12) is holomorphic on $\mathcal{R}(\mathbb{C} \backslash\{0\}) \times \mathbb{D}\left(u^{0}\right)$, with holomorphic matrix coefficients $\Psi_{j}(u)$, and the series is convergent uniformly w.r.t. $u \in \mathbb{D}\left(u^{0}\right)$. Moreover, $G^{(0)}(u)$ is a holomorphic fundamental solution of the integrable Pfaffian system

$$
d G=\left(\sum_{j=1}^{n} \omega_{k}(u) \mathrm{d} u_{k}\right) G,
$$

and $A(u)$ is holomorphically similar to the Jordan form $J=G^{(0)}(u)^{-1} A(u) G^{(0)}(u)$.

The above theorem is analogous to the characterization of isomonodromic deformations in [33], but includes also possible resonances in $A$ (see [13] and Appendix B of [25]). Notice that $\omega(z, u)$ in (2.14)-(2.15) has components

$$
\omega_{k}(u)=\left(\frac{A_{i j}\left(\delta_{i k}-\delta_{j k}\right)}{u_{i}-u_{j}}\right)_{i, j=1}^{n}=\left(\begin{array}{ccccc}
0 & 0 & \frac{-A_{1 k}}{u_{1}-u_{k}} & 0 & 0 \\
0 & 0 & \vdots & 0 & 0 \\
\frac{A_{k 1}}{u_{k}-u_{1}} & \cdots & 0 & \cdots & \frac{A_{k n}}{u_{k}-u_{n}} \\
0 & 0 & \vdots & 0 & 0 \\
0 & 0 & \frac{-A_{n k}}{u_{n}-u_{k}} & 0 & 0
\end{array}\right)
$$

Notice that $B=\operatorname{diag}(A(u))=\operatorname{diag}\left(\lambda_{1}^{\prime}, \ldots, \lambda_{n}^{\prime}\right)$ is constant because (2.16) and (2.18) imply

$$
\frac{\partial A_{i i}}{\partial u_{j}}=0, \quad \forall i, j=1, \ldots, n .
$$

\subsubsection{Deformations in $\mathbb{D}\left(u^{c}\right)$ with coalescences}

When the polydisc contains a coalescence locus $\Delta$, the analysis presents problematic issues.

- A fundamental matrix solution $Y(z, u)$ holomorphic on $\left.\mathcal{R}\left((\mathbb{C} \backslash\{0\}) \times\left(\mathbb{D}\left(u^{c}\right)\right) \backslash \Delta\right)\right)$ may be singular at $\Delta$, namely the limit for $u \rightarrow u^{*} \in \Delta$ along any direction may diverge, and $\Delta$ is in general a branching locus [43].

- The monodromy data associated with a fundamental matrix solution $\stackrel{Y}{Y}(z)$ of

$$
\frac{\mathrm{d} Y}{\mathrm{~d} z}=\left(\Lambda\left(u^{c}\right)+\frac{A\left(u^{c}\right)}{z}\right) Y,
$$

differ from those of any fundamental solution $Y(z, u)$ of $(1.1)$ at $u \notin \Delta([2,3,13])$.

In $\mathcal{R}(\mathbb{C} \backslash\{0\})$, we introduce the Stokes rays of $\Lambda\left(u^{c}\right)$

$$
\Re\left(\left(u_{i}^{c}-u_{k}^{c}\right) z\right)=0, \quad \Im\left(\left(u_{i}^{c}-u_{k}^{c}\right) z\right)<0, \quad u_{i} \neq u_{k},
$$


and an admissible direction at $u^{c}$

$$
\arg z=\tau,
$$

such that none of the Stokes rays at $u=u^{c}$ take this direction. Notice that $\tau$ is associated with $u^{c}$, differently from $\tau^{(0)}$ of Sect. 2.1.1. We choose $\mu$ basic Stokes rays of $\Lambda\left(u^{c}\right)$. These are all and the only Stokes rays lying in a sector of amplitude $\pi$, whose boundaries are not Stokes rays of $\Lambda\left(u^{c}\right)$. Notice that $\mu$ is different from $\mu^{(0)}$ used in Sect. 2.1.1. We label their directions $\arg (z)$ as follows:

$$
\tau_{0}<\tau_{1}<\cdots<\tau_{\mu-1}
$$

The directions of all the other Stokes rays of $\Lambda\left(u^{c}\right)$ in $\mathcal{R}(\mathbb{C} \backslash\{0\})$ are consequently labelled by an integer $v \in \mathbb{Z}$

$$
\arg z=\tau_{v}:=\tau_{\nu_{0}}+k \pi, \quad \text { with } \nu_{0} \in\{0, \ldots, \mu-1\} \text { and } v:=v_{0}+k \mu \text {. }
$$

They satisfy $\tau_{v}<\tau_{v+1}$.

Analogously, at any other $u \in \mathbb{D}\left(u^{c}\right)$, we define Stokes rays $\Re\left(\left(u_{i}-u_{j}\right) z\right)=0$, $\Im\left(\left(u_{i}-u_{j}\right) z\right)<0$ of $\Lambda(u)$. They behave differently from the case of $\mathbb{D}\left(u^{0}\right)$. Indeed, if $u$ varies in $\mathbb{D}\left(u^{c}\right)$, some Stokes rays cross the admissible directions $\arg z=\tau \bmod$ $\pi$, as follows. Let $i, j, k$ be such that $u_{i}^{c}=u_{j}^{c} \neq u_{k}^{c}$. Then, as $u$ moves away from $u^{c}$, a Stokes ray of $\Lambda\left(u^{c}\right)$ characterized by $\Re\left(\left(u_{i}^{c}-u_{k}^{c}\right) z\right)=0$ generates three rays. Two of them are $\Re\left(\left(u_{i}-u_{k}\right) z\right)=0$ and $\Re\left(\left(u_{j}-u_{k}\right) z\right)=0$. If $\mathbb{D}\left(u^{c}\right)$ is sufficiently small (as in (5.1)), they do not cross $\arg z=\tau \bmod \pi$ as $u$ varies in $\mathbb{D}\left(u^{c}\right)$. The third ray is $\mathfrak{R}\left(\left(u_{i}-u_{j}\right) z\right)=0$. When $u$ varies in $\mathbb{D}\left(u^{c}\right)$ making a complete loop $\left(u_{i}-u_{j}\right) \mapsto\left(u_{i}-u_{j}\right) e^{2 \pi i}$ around the locus $\left\{u \in \mathbb{D}\left(u^{c}\right) \mid u_{i}-u_{j}=0\right\} \subset \Delta$, the third ray crosses $\arg z=\tau \bmod 2 \pi$ and $\arg z=\tau-\pi \bmod 2 \pi$. This identifies a crossing locus $X(\tau) \subset \mathbb{D}\left(u^{c}\right)$ of points $u$ such that there exists a Stokes ray of $\Lambda(u)$ (so infinitely many in $\mathcal{R}(\mathbb{C} \backslash\{0\})$ ) with direction $\tau \bmod \pi$.

Proposition 2.2 ([13]). Each connected component of $\mathbb{D}\left(u^{c}\right) \backslash(\Delta \cup X(\tau))$ is simply connected and homeomorphic to a ball, so it is a topological cell.

Thus, the choice of $\tau$ induces a cell decomposition of $\mathbb{D}\left(u^{c}\right)$. Each cell is called $\tau$-cell. If $u$ varies in the interior of a $\tau$-cell, no Stokes rays cross the admissible directions $\arg z=\tau+h \pi, h \in \mathbb{Z}$, but if $u$ varies in the whole $\mathbb{D}\left(u^{c}\right)$, then $X(\tau)$ is crossed, and thus Proposition 2.1 does not hold.

To overcome this difficulty, we first take a point $u^{0}$ in a $\tau$-cell, and consider a polydisc $\mathbb{D}\left(u^{0}\right)$ contained in the $\tau$-cell, satisfying the assumptions of Sect. 2.1.1. Accordingly, we can define as before the sectors $\mathcal{S}_{v+k \mu}(u)$ of angular amplitude greater than $\pi$, and

$$
\mathcal{S}_{\nu+k \mu}\left(\mathbb{D}\left(u^{0}\right)\right)=\bigcap_{u \in \mathbb{D}\left(u^{0}\right)} \mathcal{S}_{\nu+k \mu}(u) \subset\left\{\tau_{\nu+k \mu}-\pi<\arg z<\tau_{\nu+k \mu+1}\right\} .
$$

Notice that here we are using $\tau$ and $\mu$ in place of $\tau^{(0)}$ and $\mu^{(0)}$. With the above sectors, monodromy data in (2.11)-(2.13) can be defined in $\mathbb{D}\left(u^{0}\right)$. 
Since $A(u)$ is holomorphic in $\mathbb{D}\left(u^{0}\right)$, then $\omega_{k}(z, u)$ is holomorphic on $\mathbb{D}\left(u^{c}\right) \backslash \Delta$. Thus, the fundamental matrix solutions $Y_{v}(z, u), Y^{(0)}(z, u)$ of Sect. 2.1.1 extend analytically on $\left.\left.\mathcal{R}\left((\mathbb{C} \backslash\{0\}) \times\left(\mathbb{D}\left(u^{c}\right)\right) \backslash \Delta\right)\right) \neq \mathcal{R}\left(\mathbb{C}_{z} \backslash\{0\}\right) \times\left(\mathbb{D}\left(u^{c}\right)\right) \backslash \Delta\right)$, and $\Delta$ may be a branching locus for them.

Proposition 2.3 ([13]). $\omega(z, u)$ in (2.15) and (2.18) is holomorphic on the whole $\mathbb{D}\left(u^{c}\right)$ if and only if

$$
A_{i j}(u)=\mathcal{O}\left(u_{i}-u_{j}\right) \rightarrow 0 \quad \text { whenever }\left(u_{i}-u_{j}\right) \rightarrow 0 \text { for } u \text { approaching } \Delta .
$$

$A(u)$ is holomorphically similar on $\mathbb{D}\left(u^{c}\right)$ to a Jordan form $J$ if and only if the above vanishing conditions hold. Similarity is realized by a fundamental matrix solution of (2.17), which exists holomorphic on the whole $\mathbb{D}\left(u^{c}\right)$.

The extension of the theory of isomonodromy deformations on the whole $\mathbb{D}\left(u^{c}\right)$ is given in [13] by the following theorem, which is a detailed exposition of the points (I) and (II) of Introduction, while point (III) is expressed by Corollary 2.1.

Theorem 2.2 ([13]). Let $A(u)$ be holomorphic on $\mathbb{D}\left(u^{c}\right)$. Assume that system (1.1) is strongly isomonodromic on $\mathbb{D}\left(u^{0}\right)$ contained in a $\tau$-cell of $\mathbb{D}\left(u^{c}\right)$, so that Theorem 2.1 holds. Moreover, assume that A satisfies the vanishing conditions

$$
A_{i j}(u)=\mathcal{O}\left(u_{i}-u_{j}\right) \rightarrow 0 \quad \text { whenever }\left(u_{i}-u_{j}\right) \rightarrow 0 \text { for } u \text { approaching } \Delta
$$

Then, the following statements hold.

\section{Part I.}

$(\mathrm{I}, 1) Y^{(0)}(z, u)$ and the $Y_{v}(z, u), v \in \mathbb{Z}$ admit analytic continuation as holomorphic functions on $\mathcal{R}(\mathbb{C} \backslash\{0\}) \times \mathbb{D}\left(u^{c}\right)$. The coalescence locus $\Delta$ is neither a singularity locus nor a branching locus.

$(\mathrm{I}, 2)$ The coefficients $F_{k}(u)$ of $Y_{F}(z, u)$, given in (2.8)-(2.9)-(2.10), are holomorphic of $u \in \mathbb{D}\left(u^{c}\right)$.

(I,3) The fundamental matrix solutions $Y_{v}(z, u)$ have asymptotics $Y_{v}(z, u) \sim Y_{F}(z, u)$ uniformly in $u \in \mathbb{D}\left(u^{c}\right)$, for $z \rightarrow \infty$ in a wide sector $\widehat{\mathcal{S}}_{v}$ containing $\mathcal{S}_{v}\left(\mathbb{D}\left(u^{0}\right)\right)$, to be defined later in (7.3).

\section{Part II.}

(II,1) the essential monodromy data $\mathbb{S}_{v}, \mathbb{S}_{v+\mu}, B=\operatorname{diag}\left(A\left(u^{c}\right)\right), C_{v}, L, D$, initially defined on $\mathbb{D}\left(u^{0}\right)$ by relations $(2.11)-(2.13)$, are well defined and constant on the whole $\mathbb{D}\left(u^{c}\right)$. They satisfy

$$
\mathbb{S}_{v}=\stackrel{\circ}{\mathbb{S}}_{v}, \quad \mathbb{S}_{v+\mu}=\stackrel{\circ}{\mathbb{S}}_{v+\mu}, \quad L=\stackrel{\circ}{L}, \quad C_{v}=\stackrel{\circ}{C}_{\nu}, \quad D=\stackrel{\circ}{D}
$$

where

(II,2) $\stackrel{\circ}{\mathbb{S}}_{v}, \stackrel{\circ}{\mathbb{S}}_{v+\mu}$ are the Stokes matrices of fundamental solutions $\stackrel{\circ}{V}_{v}(z), \stackrel{\circ}{Y}_{v+\mu}(z)$, $\stackrel{\circ}{Y}_{v+2 \mu}(z)$ of (2.19) having asymptotic behaviour $\stackrel{\circ}{F}_{F}(z)=Y_{F}\left(z, u^{c}\right)$, for $z \rightarrow \infty$, respectively, on sectors $\tau_{v}-\pi<\arg z<\tau_{v+1}, \tau_{v}<\arg z<\tau_{v+\mu+1}$ and $\tau_{\nu+\mu}<\arg z<\tau_{\nu+2 \mu+1}$ 
(II,3) $\stackrel{\circ}{L}, \stackrel{\circ}{D}$ are the exponents of a fundamental solution $\stackrel{\circ}{Y}(z)=\stackrel{\circ}{G}\left(I+\sum_{j=1}^{\infty} \stackrel{\circ}{\Psi}_{j} z^{j}\right) z^{\stackrel{\circ}{D}}$ $z^{\stackrel{\circ}{L}}$ of (2.19) in Levelt form;

(II,4) $\stackrel{\circ}{C}_{v}$ connects $\stackrel{\circ}{Y}_{v}(z)=\stackrel{\circ}{Y}(z) \stackrel{\circ}{C}_{v}$.

(II,5) The Stokes matrices satisfy the vanishing conditions

$$
\begin{aligned}
& \left(\mathbb{S}_{v}\right)_{i j}=\left(\mathbb{S}_{v}\right)_{j i}=0, \quad\left(\mathbb{S}_{\nu+\mu}\right)_{i j}=\left(\mathbb{S}_{\nu+\mu}\right)_{j i}=0 \\
& \forall 1 \leq i \neq j \leq n \text { such that } u_{i}^{c}=u_{j}^{c} .
\end{aligned}
$$

Corollary 2.1 ([13]). If $A_{i i}-A_{j j} \notin \mathbb{Z} \backslash\{0\}$ for every $i \neq j$ such that $u_{i}^{c}=u_{j}^{c}$, then the formal solution $\stackrel{\circ}{Y}_{F}(z)$ of (2.19) is unique and coincides with $Y_{F}\left(z, u^{c}\right)$.

The assumption of Corollary 2.1 will be called partial non-resonance. If it holds, (II,1) says that in order to obtain the essential monodromy data of (1.1) it suffices to compute $\stackrel{\circ}{S}_{v}, \stackrel{\circ}{S}_{v+\mu}, \stackrel{\circ}{L}, \stackrel{\circ}{C}_{v}$ and $\stackrel{\circ}{D}$ for (2.19), which is simper than (1.1), because $A_{i j}\left(u^{c}\right)=0$ for $i, j$ such that $u_{i}^{c}=u_{j}^{c}$. This allows in some cases the explicit computation of monodromy data. An important example with algebro-geometric implications can be found in [14].

Remark 2.1 The following statement, not mentioned in [13], holds.

If (1.1) is an isomonodromic family on the polydisc minus the coalescence locus, in the sense that $d Y=\omega Y$ in (2.14)-(2.15) is Frobenius integrable on $\mathbb{D}\left(u^{c}\right) \backslash \Delta$, and if $A(u)$ is holomorphic on $\mathbb{D}\left(u^{c}\right)$, then the vanishing conditions (2.22) hold automatically and (1.1) is isomonodromic on $\mathbb{D}\left(u^{c}\right)$ in the strong sense, namely Theorem 2.2 holds.

I thank the referee for suggesting to write the above statement. The sketch of the proof is as follows: integrability $\mathrm{d} \omega=\omega \wedge \omega$ on $\mathbb{D}\left(u^{c}\right) \backslash \Delta$ implies (2.16), namely

$$
\frac{\partial A}{\partial u_{j}}=\left[\omega_{j}(u), A\right], \quad j=1, \ldots, n ; \quad u \in \mathbb{D}\left(u^{c}\right) \backslash \Delta .
$$

We want to prove that $A_{i j}(u) \rightarrow 0$ for $u_{i}-u_{j} \rightarrow 0$, for $i \neq j$. From (2.23) and (2.18) we explicitly obtain

$$
\frac{\partial A_{i \ell}}{\partial u_{j}}=\frac{\left(u_{i}-u_{\ell}\right) A_{i j} A_{j \ell}}{\left(u_{i}-u_{j}\right)\left(u_{\ell}-u_{j}\right)}, \quad \text { for } j \neq i, \ell \text { and } i \neq \ell .
$$

The left-hand side is holomorphic everywhere on $\mathbb{D}\left(u^{c}\right)$ by assumption on $A$, and so must be the right-hand side. This implies that holomorphically $A_{i j}=O\left(u_{i}-u_{j}\right)$ for $u_{i}-u_{j} \rightarrow 0$. Then, Theorem 2.2 holds and we conclude.

The difficulty in proving Theorem 2.2 is the analysis of the Stokes phenomenon at $z=\infty$. On the other hand, coalescences does not affect the analysis at the Fuchsian singularity $z=0$, so it is not an issue for the proof of the statements concerning $Y^{(0)}(z, u), L, D$ and $C_{v}$ (as far as the contribution of $Y^{(0)}$ is concerned). See Proposition 17.1 of [13], and the proof of Theorem 4.9 in [25]. For this reason, in the present paper we will not deal with $Y^{(0)}(z, u), L, D, C_{v}$ and $(I I, 3)-(I I, 4)$ above. 
In Theorem 7.1 we introduce an isomonodromic Laplace transform in order to prove the statements of Theorem 2.2 above, concerning the Stokes phenomenon, namely $(I, 1)$, $(I, 2),(I, 3)$ and $(I I, 1),(I I, 2),(I I, 5)$.

\subsection{Background 2: Laplace transform, connection coefficients and Stokes matrices}

In this section, we fix $u \in \mathbb{D}\left(u^{c}\right) \backslash \Delta$. Accordingly, system (1.1) is to be considered as a system not depending on deformation parameters, with leading matrix $\Lambda$ having pairwise distinct eigenvalues, and system (1.4) is equivalent to (1.3), which does not depend on parameters. For simplicity of notations, let us fix for example

$$
u=u^{0}, \quad \text { as in Section 2.1.1. }
$$

Solutions $Y_{v}(z)$ of (1.1) with canonical asymptotics $Y_{F}(z)\left(u=u^{0}\right.$ fixed is not indicated) can be expressed in terms of convergent Laplace-type integrals [5,31], where the integrands are solutions of the Fuchsian system ${ }^{5}$

$$
(\Lambda-\lambda) \frac{\mathrm{d} \Psi}{\mathrm{d} \lambda}=(A+I) \Psi, \quad I:=\text { identity matrix }
$$

Indeed, let $\vec{\Psi}(\lambda)$ be a vector valued function and define

$$
\vec{Y}(z)=\int_{\gamma} e^{\lambda z} \vec{\Psi}(\lambda) \mathrm{d} \lambda
$$

where $\gamma$ is a suitable path. Then, substituting in (1.1), we have

$$
(z \Lambda+A) \int_{\gamma} e^{\lambda z} \vec{\Psi}(\lambda) \mathrm{d} \lambda=z \frac{\mathrm{d}}{\mathrm{d} z} \int_{\gamma} e^{\lambda z} \vec{\Psi}(\lambda) \mathrm{d} \lambda=z \int_{\gamma} \lambda e^{\lambda z} \vec{\Psi}(\lambda) \mathrm{d} \lambda
$$

This implies that

$$
\begin{aligned}
A \int_{\gamma} e^{\lambda z} \vec{\Psi}(\lambda) \mathrm{d} \lambda & =\int_{\gamma} \frac{\mathrm{d}\left(e^{\lambda z}\right)}{\mathrm{d} \lambda}(\lambda-\Lambda) \vec{\Psi}(\lambda) \mathrm{d} \lambda \\
& =\left.e^{\lambda z}(\lambda-\Lambda) \vec{\Psi}(\lambda)\right|_{\gamma}-\int_{\gamma} e^{\lambda z}\left[(\lambda-\Lambda) \frac{\mathrm{d} \vec{\Psi}(\lambda)}{\mathrm{d} \lambda}+\vec{\Psi}(\lambda)\right] \mathrm{d} \lambda
\end{aligned}
$$

If $\gamma$ is such that $\left.e^{\lambda z}(\lambda-\Lambda) \vec{\Psi}(\lambda)\right|_{\gamma}=0$, and if the function $\vec{\Psi}(\lambda)$ solves (2.24), then $\vec{Y}(z)$ solves (1.1).

\footnotetext{
5 The notation $A_{0}$ and $A_{1}$ is used in [23] for $\Lambda$ and $A$. In [4] the notation for $\Lambda$ is the same, while $A$ is denoted by $A_{1}$. The notation $\lambda_{1}, \ldots, \lambda_{n}$ is used in $[4,25]$ for $u_{1}, \ldots, u_{n}$. There is a misprint in the first page of [23] where it is said that $A_{1} \in G L(n, \mathbb{C})$; the correct statement is $A_{1} \in \operatorname{Mat}(n, \mathbb{C})$. 
Multiplying to the left by $(\Lambda-\lambda)^{-1}$, system (2.24) becomes (1.3),

$$
\frac{\mathrm{d} \Psi}{\mathrm{d} \lambda}=\sum_{k=1}^{n} \frac{B_{k}}{\lambda-u_{k}^{0}} \Psi, \quad B_{k}:=-E_{k}(A+I) .
$$

In order to define matrix solutions of of (2.26) as single valued functions, we consider the $\lambda$-plane with branch-cuts. Let $\eta^{(0)} \in \mathbb{R}$ satisfy

$$
\eta^{(0)} \neq \arg \left(u_{j}^{0}-u_{k}^{0}\right) \bmod \pi, \quad \text { for all } 1 \leq j, k \leq n .
$$

We fix parallel cuts $L_{k}\left(\eta^{(0)}\right)$, namely half-lines oriented from $u_{k}^{0}$ to $\infty$ in direction $\arg \left(\lambda-u_{k}^{0}\right)=\eta^{(0)}, 1 \leq k \leq n$. See Fig. 2. Conditions (2.27) mean that a cut $L_{k}$ does not contain another pole $u_{j}^{0}, j \neq k$. For this reason $\eta^{(0)}$ is called admissible direction at $u^{0}$. Then, we choose a branch of the logarithms $\ln \left(\lambda-u_{k}^{0}\right)=\ln \left|\lambda-u_{k}^{0}\right|+i \arg \left(\lambda-u_{k}^{0}\right)$ by

$$
\eta^{(0)}-2 \pi<\arg \left(\lambda-u_{k}^{0}\right)<\eta^{(0)}, \quad k=1, \ldots, n .
$$

Following [4], the $\lambda$-plane with these cuts and choices of the logarithms is denoted by $\mathcal{P}_{\eta^{(0)}}$. Matrix solutions of (2.26) are well defined as single-valued functions of $\lambda \in \mathcal{P}_{\eta^{(0)}}$.

Remark 2.2 $\mathcal{P}_{\eta^{(0)}}$ can be identified with one of the countably many components of

$$
\mathcal{R}^{\prime}:=\mathcal{R}\left(\mathbb{C} \backslash\left\{u_{1}^{0}, \ldots, u_{n}^{0}\right\}\right)-\left(\text { lift of all half-lines } L_{k}\right)
$$

Each component is obtained by a deck transformation starting from one. Fix one component, for example $\mathcal{P}_{\eta^{(0)}}$, and define $2 n$ letters

$$
\begin{aligned}
& l_{k}:=\text { cross a lift of } L_{k} \text { from the right, } \\
& l_{k}^{-1}:=\text { cross a lift of } L_{k} \text { from the left, } k=1, \ldots, n,
\end{aligned}
$$

where "right" or "left" refers to the orientation of $L_{k}$. The other components are reached by crossing the cuts, so that there is a one to one correspondence between finite sequences $\left\{l_{j_{1}}^{ \pm 1}, \ldots, l_{j_{m}}^{ \pm 1}\right\}$ not containing successively a $l_{k}^{ \pm 1}$ and $l_{k}^{\mp 1}$, and components of $\mathcal{R}^{\prime}$ (here $j_{1}, \ldots, j_{m} \in\{1, \ldots, n\}$ and $m \in \mathbb{N}$ ). The relations (2.28) alone do not identify a component of $\mathcal{R}^{\prime}$ (as incorrectly written in [23], page 387). For example, the word $l_{1} l_{2} l_{1}^{-1} l_{2}^{-1}$ leads to a new component of $\mathcal{R}^{\prime}$ where $\arg \left(\lambda-u_{1}^{0}\right), \ldots, \arg \left(\lambda-u_{n}^{0}\right)$ take the same values of the starting component. ${ }^{6}$ I thank the referee for this remark.

Stokes matrices for (1.1), for fixed and pairwise distinct $u_{1}^{0}, \ldots, u_{n}^{0}$, can been expressed in terms of connection coefficients of selected solutions of (2.26). The

\footnotetext{
6 As well known, the analytic continuation, starting from the plane $\mathcal{P}_{\eta^{(0)}}$, of a fundamental matrix solution of (2.26) defines a function $\Psi$ on $\mathcal{R}\left(\mathbb{C} \backslash\left\{u_{1}^{0}, \ldots, u_{n}^{0}\right\}\right)$. For example, if $\lambda^{\prime}$ is the lift of $\lambda \in \mathcal{P}_{\eta^{(0)}}$ to the component of $\mathcal{R}^{\prime}$ identified by the word $l_{1} l_{2} l_{1}^{-1} l_{2}^{-1}$, then $\Psi\left(\lambda^{\prime}\right)=\Psi(\lambda) M_{2}^{-1} M_{1}^{-1} M_{2} M_{1}$, where $M_{j}$ is the monodromy matrix associated with $l_{j}$.
} 
explicit relations have been obtained in [4] for the generic case when all $\lambda_{1}^{\prime}, \ldots, \lambda_{n}^{\prime} \notin$ $\mathbb{Z}$; and in [23] for the general case with no restrictions on $\lambda_{1}^{\prime}, \ldots, \lambda_{n}^{\prime}$ and $A$.

\section{Selected vector solutions}

The Laplace transform involves three types of vector solutions of (2.26), denoted in [23], respectively, by $\vec{\Psi}_{k}(\lambda), \vec{\Psi}_{k}^{*}(\lambda)$ and $\vec{\Psi}_{k}^{(\text {sing })}(\lambda)$, for $k=1, \ldots, n$ (in [4] the notation used is $Y_{k}$ and $Y_{k}^{*}$, while $Y_{k}^{(s i n g)}$ does not appear, since it reduces to $Y_{k}$ in the generic case $\lambda_{k}^{\prime} \notin \mathbb{Z}$ ). We will not describe here the $\vec{\Psi}_{k}^{*}(\lambda)$, which play mostly a technical role. Let

$$
\begin{aligned}
& \mathbb{N}=\{0,1,2, \ldots\} \text { integers, } \mathbb{Z}_{-}=\{-1,-2,-3, \ldots\} \text { negative integers, } \\
& \vec{e}_{k}=\text { standard } k \text { th unit column vector in } \mathbb{C}^{n} .
\end{aligned}
$$

It is proved in [23] that for every $k \in\{1, \ldots, n\}$ there are at least $n-1$ independent vector solutions holomorphic at $\lambda=u_{k}^{0}$. The remaining independent solution is singular at $\lambda=u_{k}^{0}$, except for some exceptional cases possibly occurring when $\lambda_{k}^{\prime} \leq-2$ is integer. In such cases, there exist $n$ holomorphic solutions at $\lambda=u_{k}^{0}$ (such cases never occur if none of the eigenvalues of $A$ is a negative integer). The selected vector solutions $\vec{\Psi}_{k}$ are obtained as follows.

- If $\lambda_{k}^{\prime} \leq-2$ is integer and we are in an exceptional case when there are no singular solutions at $u_{k}^{0}$, namely

$$
\vec{\Psi}_{k}^{(\operatorname{sing})}(\lambda) \equiv 0
$$

then $\vec{\Psi}_{k}$ is the unique analytic solution with the following normalization:

$$
\vec{\Psi}_{k}(\lambda)=\left(\frac{(-1)^{\lambda_{k}^{\prime}}}{\left(-\lambda_{k}^{\prime}-1\right) !} \vec{e}_{k}+\sum_{l \geq 1} \vec{b}_{l}^{(k)}\left(\lambda-u_{k}^{0}\right)^{l}\right)\left(\lambda-u_{k}^{0}\right)^{-\lambda_{k}^{\prime}-1} .
$$

- In all other cases, there is a solution $\vec{\Psi}_{k}^{(\text {sing })}$ with singular behaviour at $\lambda=u_{k}^{0}$. This is determined up to a multiplicative factor and the addition of an arbitrary linear combination of the remaining $n-1$ regular at $\lambda=u_{k}^{0}$ solutions, denoted below with $\operatorname{reg}\left(\lambda-u_{k}^{0}\right)$. In [23], it has the following structure

$$
\vec{\Psi}_{k}^{(\text {sing })}(\lambda)=\left\{\begin{array}{cc}
\vec{\psi}_{k}(\lambda)\left(\lambda-u_{k}^{0}\right)^{-\lambda_{k}^{\prime}-1}+\operatorname{reg}\left(\lambda-u_{k}^{0}\right), & \lambda_{k}^{\prime} \notin \mathbb{Z}, \\
\vec{\psi}_{k}(\lambda) \ln \left(\lambda-u_{k}^{0}\right)+\operatorname{reg}\left(\lambda-u_{k}^{0}\right), & \lambda_{k}^{\prime} \in \mathbb{Z}_{-}, \\
\frac{P_{k}(\lambda)}{\left(\lambda-u_{k}^{0}\right)^{\lambda_{k}^{\prime}+1}}+\vec{\psi}_{k}(\lambda) \ln \left(\lambda-u_{k}^{0}\right)+\operatorname{reg}\left(\lambda-u_{k}^{0}\right), & \lambda_{k}^{\prime} \in \mathbb{N} .
\end{array}\right.
$$


Here $\vec{\psi}_{k}(\lambda)$ is analytic at $u_{k}^{0}$ and $P_{k}(\lambda)=\sum_{l=0}^{\lambda_{k}^{\prime}} \vec{b}_{l}^{(k)}\left(\lambda-u_{k}^{0}\right)^{l}$ is a polynomial of degree $\lambda_{k}^{\prime}$. We choose the following normalization at $\lambda=u_{k}^{0}$

$$
\left\{\begin{array}{cc}
\vec{\psi}_{k}(\lambda)=\Gamma\left(\lambda_{k}^{\prime}+1\right) \vec{e}_{k}+\sum_{l \geq 1} \vec{b}_{l}^{(k)}\left(\lambda-u_{k}^{0}\right)^{l}, & \lambda_{k}^{\prime} \notin \mathbb{Z}, \\
\vec{\psi}_{k}(\lambda)=\left(\frac{(-1)^{\lambda_{k}^{\prime}}}{\left(-\lambda_{k}^{\prime}-1\right) !} \vec{e}_{k}+\sum_{l \geq 1} \vec{b}_{l}^{(k)}\left(\lambda-u_{k}^{0}\right)^{l}\right)\left(\lambda-u_{k}^{0}\right)^{-\lambda_{k}^{\prime}-1} \lambda_{k}^{\prime} \in \mathbb{Z}_{-}, \\
P_{k}(\lambda)=\lambda_{k}^{\prime} ! \vec{e}_{k}+O\left(\lambda-u_{k}^{0}\right) & \lambda_{k}^{\prime} \in \mathbb{N},
\end{array}\right.
$$

The coefficients $\vec{b}_{l}^{(k)} \in \mathbb{C}^{n}$ are uniquely determined by the normalization. Then the selected vector solutions $\vec{\Psi}_{k}$ are uniquely defined by ${ }^{7}$

$$
\vec{\Psi}_{k}(\lambda):=\vec{\psi}_{k}(\lambda)\left(\lambda-u_{k}^{0}\right)^{-\lambda_{k}^{\prime}-1} \text { for } \lambda_{k}^{\prime} \notin \mathbb{Z} ; \quad \vec{\Psi}_{k}(\lambda):=\vec{\psi}_{k}(\lambda) \text { for } \lambda_{k}^{\prime} \in \mathbb{Z}
$$

In case $\lambda_{k}^{\prime} \in \mathbb{N}$, depending on the system, it may exceptionally happen that

$$
\vec{\Psi}_{k}:=\vec{\psi}_{k} \equiv 0
$$

Remark 2.3 Suppose $\lambda_{k}^{\prime} \in \mathbb{Z}$. In particular, if $\lambda_{k}^{\prime} \leq-2$, suppose we are in the case when $\vec{\Psi}_{k}^{(\text {sing })}$ is not identically zero. Then

$$
\vec{\Psi}_{k}(\lambda)=\frac{1}{2 \pi i}\left(\vec{\Psi}_{k}^{(\text {sing })}\left(l_{k}(\lambda)\right)-\vec{\Psi}_{k}^{(\text {sing })}(\lambda)\right), \quad \lambda \in \mathcal{P}_{\eta^{(0)}},
$$

is the difference of two singular solutions defined on $\mathcal{P}_{\eta^{(0)}}$. Here, in the notation of Remark 2.2, the function $\vec{\Psi}_{k}^{(\text {sing })}\left(l_{k}(\lambda)\right)$ is the value at $\lambda \in \mathcal{P}_{\eta^{(0)}}$ of the analytic continuation of $\vec{\Psi}_{k}^{(\text {sing) }}(\lambda)$ when passing from a prefixed component of $\mathcal{R}^{\prime}$, in this case $\mathcal{P}_{\eta^{(0)}}$, to the component associated with the sequence $\left\{l_{k}\right\}$ of only one element. Namely, the analytic continuation for a small loop $\left(\lambda-u_{k}^{(0)}\right) \longmapsto\left(\lambda-u_{k}^{(0)}\right) e^{2 \pi i}$.

\section{Connection coefficients}

Above, the behaviour of $\vec{\Psi}_{k}(\lambda)$ has been described at $\lambda=u_{k}^{0}$. The behaviour at any point $\lambda=u_{j}^{0}$, for $j=1, \ldots, n$, will be expressed by linear relations

$$
\begin{aligned}
& \vec{\Psi}_{k}(\lambda)=\vec{\Psi}_{j}^{(\operatorname{sing})}(\lambda) c_{j k}+\operatorname{reg}\left(\lambda-u_{j}^{0}\right) . \\
& c_{j k}:=0, \quad \forall k=1, \ldots, n, \quad \text { when } \vec{\Psi}_{j}^{(\text {sing })}(\lambda) \equiv 0 \text { (possible only if } \lambda_{j}^{\prime} \in-\mathbb{N}-2 \text { ). }
\end{aligned}
$$

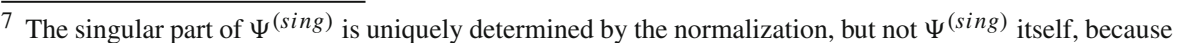
the analytic additive term $\operatorname{reg}\left(\lambda-u_{k}^{0}\right)$ is an arbitrary linear combination of the remaining $n-1$ independent analytic solutions.
} 


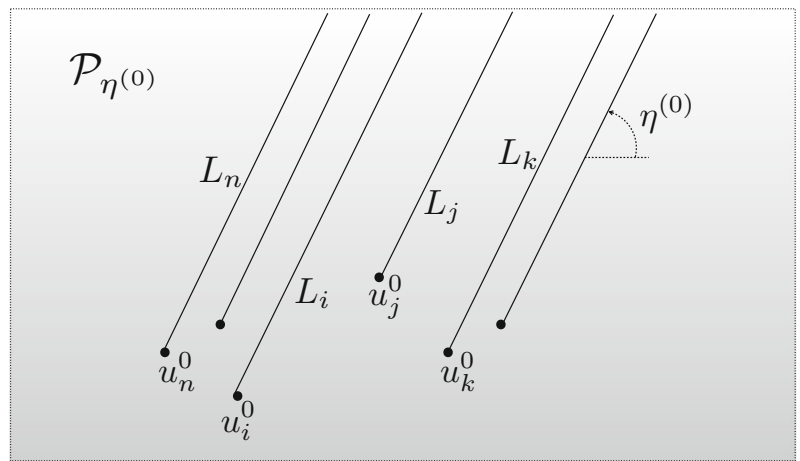

Fig. 2 The poles $u_{j}^{0}, 1 \leq j \leq n$, of system (2.26) and plane $\mathcal{P}_{\eta^{(0)}}$ with branch cuts $L_{j}$

The above relations define the connection coefficients $c_{j k}$. From the definition, we see that $c_{k k}=1$ for $\lambda_{k}^{\prime} \notin \mathbb{Z}$, while $c_{k k}=0$ for $\lambda_{k}^{\prime} \in \mathbb{Z}$. In case $\lambda_{k}^{\prime} \in \mathbb{N}$, if it happens that $\vec{\Psi}_{k} \equiv 0$, then $c_{j k}=0$ for any $j=1, \ldots, n$.

Proposition 2.4 (see [4] and Propositions 3, 4 of [23]). If A has no integer eigenvalues, then

$$
\Psi(\lambda)=\left[\vec{\Psi}_{1}(\lambda)|\cdots| \vec{\Psi}_{n}(\lambda)\right], \quad \lambda \in \mathcal{P}_{\eta^{(0)}}
$$

(each $\vec{\Psi}_{k}$ occupies a column) is a fundamental matrix solution of (2.26). Moreover, the matrix $C:=\left(c_{j k}\right)$ is invertible if and only if $A$ has no integer eigenvalues. If $A$ has integer eigenvalues and $\Psi$ is fundamental, then some $\lambda_{k}^{\prime} \in \mathbb{Z}$.

\section{Laplace transform and stokes matrices in terms of connection coefficients}

If $\eta^{(0)}$ is admissible in the $\lambda$-plane, with respect to the fixed and pairwise distinct $u_{1}^{0}, \ldots, u_{n}^{0}$, then

$$
\arg z=\tau^{(0)}:=3 \pi / 2-\eta^{(0)}
$$

is an admissible direction (2.2) in the $z$-plane for system (1.1) at the fixed $u=u^{0}$. We consider the Stokes rays of $\left.\Lambda\left(u^{0}\right)\right)$ as before. For some $v \in \mathbb{Z}$, a labelling (2.3) holds, so that

$$
\tau_{\nu}<\tau^{(0)}<\tau_{\nu+1} \quad \Longleftrightarrow \quad \eta_{\nu+1}<\eta^{(0)}<\eta_{\nu}, \quad \eta_{v}:=\frac{3 \pi}{2}-\tau_{\nu} .
$$

In order to keep track of (2.33), we label (2.32) with $v$,

$$
\Psi_{\nu}(\lambda)=\left[\vec{\Psi}_{1}(\lambda \mid v)|\cdots| \vec{\Psi}_{n}(\lambda \mid v)\right], \quad \lambda \in \mathcal{P}_{\eta^{(0)}} .
$$

The connection coefficients will be labelled accordingly as $c_{j k}^{(v)}$. Also the singular vector solutions will be labelled $\vec{\Psi}_{k}^{(\text {sing })}(\lambda \mid v), \lambda \in \mathcal{P}_{\eta^{(0)}}$ as above. 
The relation between solutions $\vec{\Psi}_{k}(\lambda \mid \nu)$ or $\vec{\Psi}_{k}^{(\operatorname{sing})}(\lambda \mid v)$ and the columns of $Y_{v}(z)$ is established in [23] for all values of $\lambda_{1}^{\prime}, \ldots, \lambda_{n}^{\prime}$, and in [4] for non integer values only. It is given by Laplace-type integrals (Proposition 8 of [23])

$$
\begin{aligned}
& \vec{Y}_{k}(z \mid v)=\frac{1}{2 \pi i} \int_{\gamma_{k}\left(\eta^{(0)}\right)} e^{z \lambda} \vec{\Psi}_{k}^{(\text {sing })}(\lambda \mid \nu) \mathrm{d} \lambda, \text { if } \lambda_{k}^{\prime} \notin \mathbb{Z}_{-} ; \\
& \vec{Y}_{k}(z \mid \nu)=\int_{L_{k}\left(\eta^{(0)}\right)} e^{z \lambda} \vec{\Psi}_{k}(\lambda \mid \nu) \mathrm{d} \lambda, \text { if } \lambda_{k}^{\prime} \in \mathbb{Z}_{-} .
\end{aligned}
$$

Here, $\gamma_{k}\left(\eta^{(0)}\right)$ is the path coming from $\infty$ along the left side of the oriented $L_{k}\left(\eta^{(0)}\right)$, encircling $u_{k}^{0}$ with a small loop excluding all the other poles, and going back to $\infty$ along the right side of $L_{k}\left(\eta^{(0)}\right)$.

The same as (2.34) can be defined for the cut-plane $\mathcal{P}_{\eta^{\prime}}$, with an admissible direction $\eta^{\prime}$ satisfying

$$
\eta_{\nu+k \mu^{(0)}+1}<\eta^{\prime}<\eta_{\mu^{(0)}+k \mu^{(0)}}, \quad k \in \mathbb{Z},
$$

and will be denoted by $\Psi_{\nu+k \mu^{(0)}}(\lambda)$, and analogously for the vectors $\vec{\Psi}_{k}\left(\lambda \mid \nu+k \mu^{(0)}\right)$ and $\vec{\Psi}_{k}^{(s i n g)}\left(\lambda \mid v+k \mu^{(0)}\right)$. From the Laplace transforms of $\vec{\Psi}_{k}\left(\lambda \mid v+k \mu^{(0)}\right)$ or $\vec{\Psi}_{k}^{(\text {sing) }}\left(\lambda \mid v+k \mu^{(0)}\right)$, with the paths of integration $\gamma_{k}\left(\eta^{\prime}\right)$ or $L_{k}\left(\eta^{\prime}\right)$, we receive $Y_{v+k \mu^{(0)}}(z)$.

Introduce in $\{1,2, \ldots, n\}$ the ordering $\prec$ given by

$$
j \prec k \Longleftrightarrow \mathfrak{R}\left(z\left(u_{j}^{0}-u_{k}^{0}\right)\right)<0 \text { for } \arg z=\tau^{(0)}, \quad i \neq j, \quad i, j \in\{1, \ldots, n\} .
$$

The following important results, proved in theorem 1 of [23] for all values of $\lambda_{1}^{\prime}, \ldots, \lambda_{n}^{\prime}$, and in the seminal paper [4] in the generic case $\lambda_{1}^{\prime}, \ldots, \lambda_{n}^{\prime} \notin \mathbb{Z}$, establish the relation between Stokes matrices and connection coefficients. ${ }^{8}$ ${ }_{8}$ The key point is the fact that $\vec{\Psi}_{k}^{(\text {sing })}$ in (7.4), or equivalently $\vec{\Psi}_{k}$ for $\lambda_{1}^{\prime}, \ldots, \lambda_{n}^{\prime} \notin \mathbb{Z}$, can be substituted
by another set of vector solutions, denoted in [23] by $\vec{\Psi}_{k}^{*}(\lambda, u \mid v)$ and in [4] by $Y_{k}^{*}$. The effect of the change
of the branch cut from $\eta_{v+1}<\eta<\eta_{v}$ to $\eta_{v+\mu+1}<\eta^{\prime}<\eta_{v+\mu}$, namely from $\eta$ to $\eta^{\prime}=\eta-\pi$, yields a
linear relation

$$
\vec{\Psi}_{k}^{*}(\lambda, u \mid v+\mu)=\vec{\Psi}_{k}^{*}(\lambda, u \mid v) C_{v}^{+}, \quad \lambda \in \mathcal{P}_{\eta} \cap \mathcal{P}_{\eta-\pi},
$$

where the connection matrix $C_{\nu}^{+}$is expressed in terms of the connection coefficients $c_{j k}^{(v)}=c_{j k}^{(v)}(\eta)$ associated with $\vec{\Psi}_{k}^{(s i n g)}(\lambda, u \mid v)$. The same can be done for the change of branch cut from $\eta_{\nu+\mu+1}<\eta^{\prime}<$ $\eta_{\nu+\mu}$ to $\eta_{\nu+2 \mu+1}<\eta^{\prime \prime}<\eta_{\nu+2 \mu}$ (namely, $\eta^{\prime}=\eta-\pi$ and $\eta^{\prime \prime}=\eta-2 \pi$ ) yielding a relation

$$
\vec{\Psi}_{k}^{*}(\lambda, u \mid v+2 \mu)=\vec{\Psi}_{k}^{*}(\lambda, u \mid v+\mu) C_{v}^{-}, \quad \lambda \in \mathcal{P}_{\eta-\pi} \cap \mathcal{P}_{\eta-2 \pi} .
$$

Substituting these relations in the Laplace integrals, one proves Theorem 2.3, being $\mathbb{S}_{v}=C_{v}^{+}$and $\mathbb{S}_{v+\mu}^{-1}=$ $C_{v}^{-}$. See [4] and [23] 
Theorem 2.3 Let $u=u^{0}$ be fixed so that $\Lambda\left(u^{0}\right)$ has pairwise distinct eigenvalues. Let $\eta^{(0)}$ and $\tau^{(0)}=3 \pi / 2-\eta^{(0)}$ be admissible for $u^{0}$ in the $\lambda$-plane and $z$-plane, respectively. Suppose that the labelling of Stokes rays is (2.3) and (2.33). Then, the Stokes matrices of system (1.1) at $u=u^{0}$ are given in terms of the connection coefficients $c_{j k}^{(v)}$ of system (2.26), according to the following formulae

$$
\begin{aligned}
\left(\mathbb{S}_{\nu}\right)_{j k}= \begin{cases}e^{2 \pi i \lambda_{k}^{\prime} \alpha_{k} c_{j k}^{(v)}} & \text { for } j \prec k, \\
1 & \text { for } j=k, \\
0 & \text { for } j \succ k,\end{cases} \\
\left(\mathbb{S}_{\nu+\mu^{(0)}}^{-1}\right)_{j k}= \begin{cases}0 & \text { for } j \prec k, \\
1 & \text { for } j=k, \\
-e^{2 \pi i\left(\lambda_{k}^{\prime}-\lambda_{j}^{\prime}\right)} \alpha_{k} c_{j k}^{(v)} & \text { for } j \succ k .\end{cases}
\end{aligned}
$$

where,

$$
\alpha_{k}:=\left(e^{-2 \pi i \lambda_{k}^{\prime}}-1\right) \quad \text { if } \lambda_{k}^{\prime} \notin \mathbb{Z} ; \quad \alpha_{k}:=2 \pi i \quad \text { if } \lambda_{k}^{\prime} \in \mathbb{Z}
$$

In the above discussion, the differential systems do not depend on parameters $(u$ is fixed). The purpose of the present paper is to extend the description of Background 2 to the case depending on deformation parameters and include coalescences in $\mathbb{D}\left(u^{c}\right)$, and then to obtain Theorem 2.2 of Background 1 in terms of an isomonodromic Laplace transform.

\section{Equivalence of the isomonodromy deformation equations for (1.1) and (1.4)}

The first step in our construction is Proposition 3.1, establishing the equivalence between strong isomonodromy deformations of systems (1.1) and (1.4), for $u$ varying in a $\tau$-cell of $\mathbb{D}\left(u^{c}\right)$. In the specific case of Frobenius manifolds, this fact can be deduced from Chapter 5 of [20]. Here we establish the equivalence in general terms.

According to Theorem 2.1, system (1.1) is strongly isomonodromic in a polydisc $\mathbb{D}\left(u^{0}\right)$ contained in a $\tau$-cell of $\mathbb{D}\left(u^{c}\right)$ if and only if ${ }^{9}$

$$
d A=\sum_{j=1}^{n}\left[\omega_{j}(u), A\right] \mathrm{d} u_{j}, \quad \omega_{j}(u)=\left[F_{1}(u), E_{j}\right], \text { given in }(2.18) .
$$

\footnotetext{
9 As already mentioned when stating Theorem 2.1, equations $d A=\left[\omega_{i}(u), A\right]$ and $\omega_{i}(u)=\left[F_{1}, E_{i}\right]$ for $i=1, \ldots, n$ are exactly the the Frobenius integrability conditions of (2.14) when (1.1) is strongly isomonodromic [13]. 
On the other hand, system (1.4) is strongly isomonodromic in $\mathbb{D}\left(u^{0}\right)$ by definition ([25], Appendix A), when fundamental matrix solutions in Levelt form at each pole $\lambda=$ $u_{j}, j=1, \ldots, n$, have constant monodromy exponents and are related to each other by constant connection matrices (not to be confused with the connection coefficients). From $[7,8,25]$, the necessary and sufficient condition for the deformation to be strongly isomonodromic (this can also be taken as the definition) is that (1.4) is the $\lambda$-component of a Frobenius integrable Pfaffian system with the following structure

$$
\mathrm{d} \Psi=P(\lambda, u) \Psi, \quad P(\lambda, u)=\sum_{k=1}^{n} \frac{B_{k}(u)}{\lambda-u_{k}} d\left(\lambda-u_{k}\right)+\sum_{k=1}^{n} \gamma_{k}(u) \mathrm{d} u_{k} .
$$

The integrability condition $d P=P \wedge P$ is the non-normalized Schlesinger system (see Appendix A and [6-8,25,27,63])

$$
\begin{aligned}
& \partial_{i} \gamma_{k}-\partial_{k} \gamma_{i}=\gamma_{i} \gamma_{k}-\gamma_{k} \gamma_{i}, \\
& \partial_{i} B_{k}=\frac{\left[B_{i}, B_{k}\right]}{u_{i}-u_{k}}+\left[\gamma_{i}, B_{k}\right], \quad i \neq k \\
& \partial_{i} B_{i}=-\sum_{k \neq i} \frac{\left[B_{i}, B_{k}\right]}{u_{i}-u_{k}}+\left[\gamma_{i}, B_{i}\right]
\end{aligned}
$$

Proposition 3.1 The system (3.1) is equivalent to (3.3)-(3.5) if and only if

$$
\gamma_{j}(u) \equiv \omega_{j}(u) \text { as in (2.15) and (2.18), } \quad j=1, \ldots, n .
$$

Namely, (1.1) is strongly isomonodromic in a polydisc on $\mathbb{D}\left(u^{0}\right)$ contained in a $\tau$-cell if and only if (1.4) is strongly isomonodromic.

Proof See Appendix B.

\section{Schlesinger system on $\mathbb{D}\left(u^{c}\right)$ and vanishing conditions}

In this section, Proposition 4.1 , we holomorphically extend to $\mathbb{D}\left(u^{c}\right)$ the nonnormalized Schlesinger system associated with (1.4), when certain vanishing conditions (4.2) are satisfied. This is the second step to obtain the results of [13] by Laplace transform.

Lemma 4.1 Let $A(u)$ be holomorphic on $\mathbb{D}\left(u^{c}\right)$ and $B_{j}(u):=-E_{j}(A(u)+I), j=$ $1, \ldots, n$.

i) The vanishing relations

$$
\left[B_{i}(u), B_{j}(u)\right] \longrightarrow 0, \quad \text { for } u_{i}-u_{j} \rightarrow 0 \text { in } \mathbb{D}\left(u^{c}\right)
$$

hold if and only if

$$
(A(u))_{i j} \longrightarrow 0, \quad \text { for } u_{i}-u_{j} \rightarrow 0 \text { in } \mathbb{D}\left(u^{c}\right) .
$$


ii) The matrices $\omega_{k}(u)=\left[F_{1}(u), E_{k}\right]$ are holomorphic on $\mathbb{D}\left(u^{c}\right)$ if and only if (4.2) holds.

Proof Let $u^{*} \in \Delta$, so that for some $i \neq j$ it occurs that $u_{i}^{*}=u_{j}^{*}$. Since

$$
B_{j}=-E_{j}(A+I)=\left(\begin{array}{ccc}
0 & 0 & 0 \\
\vdots & \vdots & \vdots \\
-A_{j 1} \cdots-A_{j, j-1}-\lambda_{j}^{\prime}-1-A_{j, j+1} \cdots & -A_{j n} \\
\vdots & \vdots & \vdots \\
0 & 0 & 0
\end{array}\right)
$$

it is an elementary computation to check the equivalence between the relation $\left[B_{i}\left(u^{*}\right), B_{j}\left(u^{*}\right)\right]=0$ and the relation $\left(A\left(u^{*}\right)\right)_{i j}=0$. Since $\left[F_{1}(u), E_{k}\right]$ is (2.18), the statement on its analyticity is straightforward.

Proposition 4.1 Consider a Frobenius integrable Pfaffian system (3.2) on $\mathbb{D}\left(u^{0}\right)$ with

$$
B_{j}(u)=-E_{j}(A(u)+I) \quad \text { and } \quad \gamma_{j}(u) \equiv \omega_{j}(u)=\left[F_{1}(u), E_{j}\right] \text { in (2.18). }
$$

Assume that $A(u)$ is holomorphic on the whole $\mathbb{D}\left(u^{c}\right)$. Then, system (3.2) is Frobenius integrable on the whole $\mathbb{D}\left(u^{c}\right)$ with holomorphic matrix coefficients if and only if the vanishing conditions (4.2) hold.

Proof If system (3.2) is integrable on $\mathbb{D}\left(u^{c}\right)$ with holomorphic coefficients $B_{k}$ and $\gamma_{k}=\omega_{k}$, then analyticity of $\omega_{k}$ with structure (2.18) implies that (4.2) must hold, so that (4.1) holds. Notice that by (4.1), the r.h.sides of (3.4)-(3.5) are holomorphic on $\mathbb{D}\left(u^{c}\right)$. Conversely, suppose that (4.1)-(4.2) hold. By Proposition 3.1, (3.3)-(3.4)-(3.5) are equivalent in $\mathbb{D}\left(u^{0}\right)$ to

$$
d A=\sum_{j=1}^{n}\left[\omega_{j}(u), A\right] \mathrm{d} u_{j}, \quad u \in \mathbb{D}\left(u^{0}\right) .
$$

Now, the 1.h.s is well defined and holomorphic on $\mathbb{D}\left(u^{c}\right)$, because so is $A(u)$. The r.h.s. is also analytic on $\mathbb{D}\left(u^{c}\right)$, because of (4.2). Hence, the first part of the Proof of Proposition 3.1 in Appendix B works in the whole $\mathbb{D}\left(u^{c}\right)$, and so the Pfaffian system (3.2) is integrable there.

For completeness, we also state the following

Proposition 4.2 Let system (3.2) with coefficients (4.4) be integrable on $\mathbb{D}\left(u^{c}\right) \backslash \Delta$ and let the $B_{k}(u)$ be holomorphic on $\mathbb{D}\left(u^{c}\right)$. Then the vanishing conditions (4.1)-(4.2), hold and the $\omega_{k}$ are holomorphic on $\mathbb{D}\left(u^{c}\right)$. 
Proof Analogously to the proof of Proposition 3.1, we see that (3.3)-(3.4)-(3.5) on $\mathbb{D}\left(u^{c}\right) \backslash \Delta$ are equivalent to

$$
d A=\sum_{j=1}^{n}\left[\omega_{j}(u), A\right] \mathrm{d} u_{j}, \quad u \in \mathbb{D}\left(u^{c}\right) \backslash \Delta .
$$

By holomorphy of $A(u)$ on $\mathbb{D}\left(u^{c}\right)$, the r.h.s is well defined, so that also the 1.h.s. must be holomorphic on $\mathbb{D}\left(u^{c}\right)$. From (4.6) we proceed as in Remark 2.1, concluding that $A_{i j}=O\left(u_{i}-u_{j}\right) \rightarrow 0$ holomorphically for $u_{i}-u_{j} \rightarrow 0$. The proof can be done also with an argument similar to Remark 6.1.

\section{Selected vector solutions depending on parameters $u \in \mathbb{D}\left(u^{c}\right)$, Theorem 5.1}

In this section, we state one main result of the paper, Theorem 5.1, introducing the isomonodromic analogue of the selected and singular vector solutions (2.30) and (2.29). This is the third step required to obtain the results of [13] by Laplace transform.

Preliminarily, we characterize the radius $\epsilon_{0}>0$ of $\mathbb{D}\left(u^{c}\right)$ in (2.1). The coalescence point $u^{c}=\left(u_{1}^{c}, \ldots, u_{n}^{c}\right)$ contains $s<n$ distinct values, say $\lambda_{1}, \ldots, \lambda_{s}$, with algebraic multiplicities $p_{1}, \ldots, p_{s}$, respectively $\left(p_{1}+\cdots+p_{s}=n\right)$. Suppose that $\arg z=\tau$ is a direction admissible at $u^{c}$, as defined in (2.20), and let

$$
\eta=3 \pi / 2-\tau
$$

be the corresponding admissible direction in the $\lambda$-plane, where we draw parallel half lines $\mathcal{L}_{1}=\mathcal{L}_{1}(\eta), \ldots, \mathcal{L}_{s}=\mathcal{L}_{s}(\eta)$ issuing from $\lambda_{1}, \ldots, \lambda_{s}$, respectively, with direction $\eta$, as in Fig. 3. Let

$$
2 \delta_{\alpha \beta}:=\text { distance between } \mathcal{L}_{\alpha} \text { and } \mathcal{L}_{\beta} \text {, for } 1 \leq \alpha \neq \beta \leq s
$$

In formulae, $2 \delta_{\alpha \beta}=\min _{\rho>0}\left|\lambda_{\alpha}-\lambda_{\beta}+\rho e^{\sqrt{-1}(3 \pi / 2-\tau)}\right|$. Then, we require that

$$
\epsilon_{0}<\min _{1 \leq \alpha \neq \beta \leq n} \delta_{\alpha \beta}
$$

The bound (5.1) was introduced in [13] in order to prove Theorem 2.2 in Background 1. It implies properties of the Stokes rays as $u$ varies in $\mathbb{D}\left(u^{c}\right)$, described later in Sect. 7. Let

$$
\mathbb{D}_{\alpha}:=\left\{\lambda \in \mathbb{C}|| \lambda-\lambda_{\alpha} \mid \leq \epsilon_{0}\right\}, \quad \alpha=1, \ldots, s,
$$

be the disc centered a $\lambda_{\alpha}$ and radius $\epsilon_{0}$. If $u_{j}$ is such that $u_{j}^{c}=\lambda_{\alpha}$, the bound (5.1) implies that $u_{j}$ remains in $\mathbb{D}_{\alpha}$ as $u$ varies in $\mathbb{D}_{\left(u^{c}\right)}$. Clearly, $\mathbb{D}_{\alpha} \cap \mathbb{D}_{\beta}=\emptyset$. 


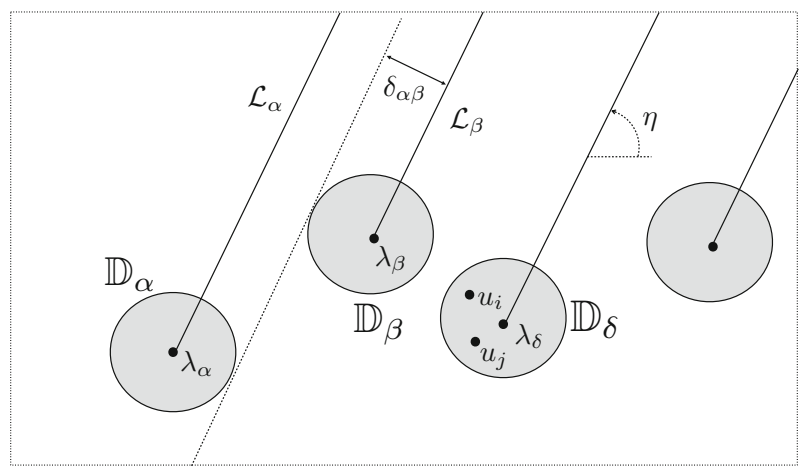

Fig. 3 The figure represents the half lines $\mathcal{L}_{\alpha}, \mathcal{L}_{\beta}$, etc, for $\alpha, \beta, \ldots \in\{1, \ldots, s\}$, in direction $\eta=3 \pi / 2-\tau$, the discs centred at the coordinates $\lambda_{1}, \ldots, \lambda_{s}$ of the coalescence point $u^{c}$, and the distances $\delta_{\alpha \beta}$. Also two points $u_{i}, u_{j}$ are represented, such that $u_{i}^{c}=u_{j}^{c}=\lambda_{\delta}$ for some $\delta \in\{1, \ldots, s\}$. Important: now $\eta$ refers to $u^{c}$, differently from Sect. 2.2 and Fig. 2

The Stokes rays of $\Lambda\left(u^{c}\right)$ can be labeled as in (2.21). For a certain $v \in \mathbb{Z}$ we have

$$
\eta_{\nu+1}<\eta<\eta_{\nu} \Longleftrightarrow \tau_{\nu}<\tau<\tau_{\nu+1}, \quad \eta_{\nu}=\frac{3 \pi}{2}-\tau_{\nu}
$$

For each $u \in \mathbb{D}\left(u^{c}\right)$, let $\mathcal{P}_{\eta}=\mathcal{P}_{\eta}(u)$ be the $\lambda$-plane with branch cuts $L_{1}=L_{1}(\eta), \ldots$, $L_{n}=L_{n}(\eta)$ issuing from $u_{1}, \ldots, u_{n}$ and the choice of the logarithms $\ln \left(\lambda-u_{k}\right)=$ $\ln \left|\lambda-u_{k}\right|+i \arg \left(\lambda-u_{k}\right)$, given by

$$
\eta-2 \pi<\arg \left(\lambda-u_{k}\right)<\eta, \quad k=1, \ldots, n .
$$

We define the domain (notation $\hat{x}$ inspired by [33])

$$
\mathcal{P}_{\eta}(u) \hat{\times} \mathbb{D}\left(u^{c}\right):=\left\{(\lambda, u) \mid u \in \mathbb{D}\left(u^{c}\right), \lambda \in \mathcal{P}_{\eta}(u)\right\},
$$

According to Proposition 4.1, for a Pfaffian system (3.2) with coefficients (4.4), defined on a polydisc $\mathbb{D}\left(u^{0}\right)$ contained in a $\tau$-cell of $\mathbb{D}\left(u^{c}\right)$, if $A(u)$ is holomorphic on $\mathbb{D}\left(u^{c}\right)$, then the vanishing conditions (4.2)

$$
(A(u))_{i j} \longrightarrow 0, \quad \text { for } u_{i}-u_{j} \rightarrow 0 \text { in } \mathbb{D}\left(u^{c}\right)
$$

are equivalent to Frobenius integrability on the whole $\mathbb{D}\left(u^{c}\right)$. With this in mind, we state the following

Theorem 5.1 Consider a Pfaffian system,

$$
\mathrm{d} \Psi=P(\lambda, u) \Psi, \quad P(z, u)=\sum_{k=1}^{n} \frac{B_{k}(u)}{\lambda-u_{k}} d\left(\lambda-u_{k}\right)+\sum_{k=1}^{n} \omega_{k}(u) \mathrm{d} u_{k}
$$


Frobenius integrable on $\mathbb{D}\left(u^{c}\right)$, with matrix coefficients (2.18), and A(u) holomorphic on $\mathbb{D}\left(u^{c}\right)$. Let the radius $\epsilon_{0}$ be as in (5.1). Then, two classes of vector solutions, holomorphic on $\mathcal{P}_{\eta}(u) \hat{\times} \mathbb{D}\left(u^{c}\right)$, exist as follows.

The selected solution: $\vec{\Psi}_{1}(\lambda, u \mid v), \ldots, \vec{\Psi}_{n}(\lambda, u \mid v)$. Each $\vec{\Psi}_{k}(\lambda, u \mid v)$ is uniquely identified by the local behaviour below for $\lambda \in \mathbb{D}_{\alpha}$, where $\alpha$ is such that $u_{k}^{c}=\lambda_{\alpha}$. The label v keeps track of (5.2).

- $\operatorname{For} \lambda_{k}^{\prime} \in \mathbb{C} \backslash \mathbb{Z}$ or $\lambda_{k}^{\prime} \in \mathbb{Z}_{-}=\{-1,-2, \ldots\}$,

$$
\vec{\Psi}_{k}(\lambda, u \mid v)=\vec{\psi}_{k}(\lambda, u \mid v)\left(\lambda-u_{k}\right)^{-\lambda_{k}^{\prime}-1}, \quad k=1, \ldots, n,
$$

where $\vec{\psi}_{k}(\lambda, u \mid v)$ is holomorphic on $\mathbb{D}_{\alpha} \times \mathbb{D}\left(u^{c}\right)$ and is represented by a uniformly convergent Taylor expansion with holomorphic on $\mathbb{D}\left(u^{c}\right)$ coefficients:

$$
\vec{\psi}_{k}(\lambda, u \mid v)=f_{k} \vec{e}_{k}+\sum_{l=1}^{\infty} \vec{b}_{l}^{(k)}(u)\left(\lambda-u_{k}\right)^{l}, \quad \text { for } \lambda \rightarrow u_{k},
$$

The following normalization uniquely identifies $\vec{\Psi}_{k}$.

$$
f_{k}=\left\{\begin{array}{cl}
\Gamma\left(\lambda_{k}^{\prime}+1\right), & \lambda_{k}^{\prime} \in \mathbb{C} \backslash \mathbb{Z} \\
\frac{(-1)^{\lambda_{k}^{\prime}}}{\left(-\lambda_{k}^{\prime}-1\right) !}, & \lambda_{k}^{\prime} \in \mathbb{Z}_{-}
\end{array}\right.
$$

- $F$ or $\lambda_{k}^{\prime} \in \mathbb{N}=\{0,1,2, \ldots\}$,

$$
\vec{\Psi}_{k}(\lambda, u \mid v)=\sum_{l=0}^{\infty} \vec{d}_{l}^{(k)}(u)\left(\lambda-u_{k}\right)^{l}, \quad \text { for } \lambda \rightarrow u_{k},
$$

is holomorphic on $\mathbb{D}_{\alpha} \times \mathbb{D}\left(u^{c}\right)$, the Taylor expansion being uniformly convergent with holomorphic coefficients $\overrightarrow{\vec{d}}_{l}^{(k)}(u)$. It is uniquely identified by the normalization (5.11) of the singular solution (5.10). Depending on the specific Pfaffian system 10 , it may happen that identically

$$
\vec{\Psi}_{k}(\lambda, u \mid v) \equiv 0
$$

The isolated singularities of $\vec{\Psi}_{k}(\lambda, u \mid \nu)$, if any, are located at $\lambda=u_{j}$ with $u_{j}^{c}=\lambda_{\beta}$, $\beta \neq \alpha$, and at $\lambda=u_{k}$ only in case $\lambda_{k}^{\prime} \in \mathbb{C} \backslash \mathbb{Z}$. For $i \neq j$ such that $u_{i}^{c}=u_{j}^{c}, \vec{\Psi}_{i}(\lambda, u \mid \nu)$ and $\vec{\Psi}_{j}(\lambda, u \mid v)$ are either linearly independent, or at least one of them is identically zero (identity to zero may occur only for $\lambda_{i}^{\prime}$ or $\lambda_{j}^{\prime}$ belonging to $\mathbb{N}$ )

10 See the comment to (6.31). 
The singular solutions: $\vec{\Psi}_{1}^{(\operatorname{sing})}(\lambda, u \mid v), \ldots, \vec{\Psi}_{n}^{(\operatorname{sing})}(\lambda, u \mid v) . \operatorname{Each} \vec{\Psi}_{k}^{(\text {sing })}(\lambda$, $u \mid v)$ is a solution with an isolated singularity at $\lambda=u_{k}$, whose singular behaviour is uniquely characterized as follows. ${ }^{11}$ Let $\mathbb{D}_{\alpha}$ be identified by $\lambda_{\alpha}=u_{k}^{c}$.

- $F$ or $\lambda_{k}^{\prime} \in \mathbb{C} \backslash \mathbb{Z}$ [algebraic or logarithmic branch-point],

$$
\vec{\Psi}_{k}^{(\text {sing })}(\lambda, u \mid v):=\vec{\Psi}_{k}(\lambda, u \mid v)=\vec{\psi}_{k}(\lambda, u \mid v)\left(\lambda-u_{k}\right)^{-\lambda_{k}^{\prime}-1}
$$

- $\operatorname{For} \lambda_{k}^{\prime} \in \mathbb{Z}_{-}[\log$ arithmic branch-point $]$,

$$
\begin{aligned}
\vec{\Psi}_{k}^{(\text {sing })}(\lambda, u \mid v)= & \vec{\Psi}_{k}(\lambda, u \mid v) \ln \left(\lambda-u_{k}\right)+\sum_{m \neq k}^{*} r_{m} \vec{\Psi}_{m}(\lambda, u \mid v) \ln \left(\lambda-u_{m}\right) \\
& +\vec{\phi}_{k}(\lambda, u \mid v), \\
& =\vec{\Psi}_{k}(\lambda, u \mid v) \ln \left(\lambda-u_{k}\right)+\operatorname{reg}\left(\lambda-u_{k}\right), \quad r_{m} \in \mathbb{C}, \quad 5
\end{aligned}
$$

where $\sum_{m \neq k}^{*}$ is over all $m$ such that $u_{m} \in \mathbb{D}_{\alpha}$ and $\lambda_{m}^{\prime} \in \mathbb{Z}_{-}$. The vector function $\vec{\phi}_{k}(\lambda, u \mid v)$ is holomorphic in $\mathbb{D}_{\alpha} \times \mathbb{D}\left(u^{c}\right)$.

In particular, for $\lambda_{k}^{\prime} \leq-2$, depending on the system, it may happen that there is no solution with singularity in $\mathbb{D}_{\alpha}$, so that

$$
\vec{\Psi}_{k}^{(\operatorname{sing})}(\lambda, u \mid \nu):=0 .
$$

- $\operatorname{For} \lambda_{k}^{\prime} \in \mathbb{N}[$ logarithmic branch-point and pole],

$$
\vec{\Psi}_{k}^{(\text {sing })}(\lambda, u \mid v)=\vec{\Psi}_{k}(\lambda, u \mid v) \ln \left(\lambda-u_{k}\right)+\frac{\vec{\psi}_{k}(\lambda, u \mid \nu)}{\left(\lambda-u_{k}\right)^{\lambda_{k}^{\prime}+1}},
$$

where $\vec{\psi}_{k}(\lambda, u \mid v)$ is holomorphic in $\mathbb{D}_{\alpha} \times \mathbb{D}\left(u^{c}\right)$,

$$
\vec{\psi}_{k}(\lambda, u \mid v)=\Gamma\left(\lambda_{k}^{\prime}+1\right) \vec{e}_{k}+\sum_{l=1}^{\infty} \vec{b}_{l}^{(k)}(u)\left(\lambda-u_{k}\right)^{l}, \quad \text { for } \lambda \rightarrow u_{i},
$$

the Taylor expansion being uniformly convergent and the coefficients $\vec{b}_{l}^{(k)}(u)$ holomorphic on $\mathbb{D}\left(u^{c}\right)$.

Let $i, j$ be such that $u_{i}^{c}=u_{j}^{c}$. Then $\vec{\Psi}_{i}^{(\text {sing })}(\lambda, u \mid v)$ and $\vec{\Psi}_{j}^{(\text {sing })}(\lambda, u \mid v)$ are either linearly independent, or at least one of them is identically zero (identity to zero can be realized only for $\lambda_{i}^{\prime} \leq-2$ or $\lambda_{j}^{\prime} \leq-2$.)

Proof See Sect. 6.

11 The solution here defined is not uniquely identified by the singular behaviour if $\lambda_{k}^{\prime} \in \mathbb{Z}_{-}$, see Remark 5.2 . 
Remark 5.1 Of the coefficients of (5.11), only $b_{0}^{(i)}(u), b_{1}^{(i)}(u), \ldots, b_{\lambda_{k}^{\prime}}^{(k)}(u)$ will be useful later.

Remark 5.2 For $\lambda_{k}^{\prime} \notin \mathbb{Z}_{-}$, the singular solution $\vec{\Psi}_{k}^{(\text {sing })}$ is unique, identified by its singular behaviour at $\lambda=u_{k}$ and the normalization (5.5)-(5.6) when $\lambda_{k}^{\prime} \in \mathbb{C} \backslash \mathbb{Z}$, or by the normalization (5.11) when $\lambda_{k}^{\prime} \in \mathbb{N}$. For $\lambda_{k}^{\prime} \in \mathbb{Z}_{-}$, a singular solution in (5.8) is not unique, but its singular behaviour (5.9) at $\lambda=u_{k}$ is uniquely fixed by the normalization (5.5)-(5.6). There is a freedom due to the choice of the coefficients $r_{m}$ and of $\vec{\phi}_{k}$ in (5.8). See also Remark 6.3.

The singular behaviour of $\vec{\Psi}_{k}$ at $\lambda=u_{j}$ is expressed by connection coefficients.

Definition 5.1 The connection coefficients are defined by

$$
\vec{\Psi}_{k}(\lambda, u \mid v) \underset{\lambda \rightarrow u_{j}}{=} \vec{\Psi}_{j}^{(\operatorname{sing})}(\lambda, u \mid v) c_{j k}^{(v)}+\operatorname{reg}\left(\lambda-u_{j}\right), \quad \lambda \in \mathcal{P}_{\eta}
$$

and by

$c_{j k}^{(v)}:=0, \forall k=1, \ldots, n, \quad$ when $\vec{\Psi}_{j}^{(\text {sing })} \equiv 0$, possibly occurring for $\lambda_{j}^{\prime} \in-\mathbb{N}-2$

The uniqueness of the singular behaviour of $\vec{\Psi}_{j}^{(\text {sing })}$ at $\lambda=u_{j}$ implies that the $c_{j k}$ are uniquely defined. From the definition, we see that

- If $\lambda_{k}^{\prime} \notin \mathbb{Z}, c_{k k}^{(v)}=1$.

- If $\lambda_{k}^{\prime} \in \mathbb{Z}, c_{k k}^{(v)}=0$.

- If $\lambda_{k}^{\prime} \in \mathbb{N}$ and $\vec{\Psi}_{k}(\lambda, u \mid v) \equiv 0$, then $c_{1 k}^{(v)}=c_{2 k}^{(v)}=\cdots=c_{n k}^{(v)}=0$.

- If $\lambda_{j}^{\prime} \in-\mathbb{N}-2$ and $\vec{\Psi}_{j}^{(\text {sing })}(\lambda, u \mid v) \equiv 0$, then $c_{j 1}^{(v)}=c_{j 2}^{(v)}=\cdots=c_{j n}^{(v)}=0$.

Proposition 5.1 The coefficients in (5.12)-(5.13) are isomonodromic connection coefficients, namely they are independent of $u \in \mathbb{D}\left(u^{c}\right)$. They satisfy the vanishing relations

$$
c_{j k}^{(v)}=0 \quad \text { for } j \neq k \text { such that } u_{j}^{c}=u_{k}^{c}
$$

Proof See Sect. 6.6.

\section{Proof of Theorem 5.1}

Remark on notations: Throughout this section, we work with functions $f=$ $f(\lambda, u \mid v)$ defined on $\mathcal{P}_{\eta}(u) \hat{\times} \mathbb{D}\left(u^{c}\right)$. For simplicity we omit $v$ and write $f=f(\lambda, u)$. Similarly, we write $c_{j k}$ in place of $c_{j k}^{(v)}$. 


\subsection{Fundamental matrix solution of the Pfaffian System}

Without loss of generality, we order the eigenvalues so that ${ }^{12}$

$$
\begin{aligned}
& u_{1}^{c}=\cdots=u_{p_{1}}^{c}=\lambda_{1} ; \quad u_{p_{1}+1}^{c}=\cdots=u_{p_{1}+p_{2}}^{c}=\lambda_{2} \\
& u_{p_{1}+p_{2}+1}^{c}=\cdots=u_{p_{1}+p_{2}+p_{3}}^{c}=\lambda_{3} ; \quad \ldots . \text { up to } u_{p_{1}+\cdots+p_{s-1}+1}^{c} \\
& \quad=\cdots=u_{p_{1}+\cdots+p_{s-1}+p_{s}}^{c}=\lambda_{s} .
\end{aligned}
$$

We analyse first the coalescence of $u_{1}, \ldots, u_{p_{1}}$ to $\lambda_{1}$. Other cases are analogous. We change variables $\left(u_{1}, \ldots, u_{n}, \lambda\right) \mapsto\left(x_{1}, \ldots, x_{n+1}\right)$ as follows

$$
x_{n+1}=\lambda-\lambda_{1}, \quad x_{j}=\left\{\begin{array}{l}
\lambda-u_{j}, \quad 1 \leq j \leq p_{1} \\
u_{j}-\lambda_{1}, p_{1}+1 \leq j \leq n .
\end{array}\right.
$$

The inverse transformation is

$$
\lambda=x_{n+1}+\lambda_{1}, \quad u_{j}=\left\{\begin{array}{l}
x_{n+1}-x_{j}+\lambda_{1}, \quad 1 \leq j \leq p_{1}, \\
x_{j}+\lambda_{1}, \quad p_{1}+1 \leq j \leq n .
\end{array}\right.
$$

Let

$$
x:=(\underbrace{x_{1}, \ldots, x_{p_{1}}}_{p_{1}}, \underbrace{x_{p_{1}+1}, \ldots, x_{n}}_{n-p_{1}}, x_{n+1}) \equiv(\underbrace{x_{1}, \ldots, x_{p_{1}}}_{p_{1}}, \boldsymbol{x}^{\prime}, x_{n+1}),
$$

where $\boldsymbol{x}^{\prime}:=\left(x_{p_{1}+1}, \ldots, x_{n}\right)$. We are interested in the behaviour of solutions for

$$
x \longrightarrow(\underbrace{0,0, \ldots, 0}_{p_{1}}, x^{\prime}, 0)
$$

corresponding to

$$
u_{1} \rightarrow \lambda_{1}, \ldots, u_{p_{1}} \rightarrow \lambda_{1}, \quad \text { and } \lambda \rightarrow \lambda_{1}
$$

namely $u_{i}-u_{j} \rightarrow 0, i \neq j$ and $\lambda-u_{i} \rightarrow 0$, for $i, j \in\left\{1, \ldots, p_{1}\right\}$. The Pfaffian system (5.3) in variables $x$, with Fuchsian singularities at $x_{1}=0, \ldots, x_{p_{1}}=0$, becomes

$$
\mathrm{d} \Psi=P(x) \Psi, \quad P(x)=\sum_{j=1}^{p_{1}} \frac{P_{j}(x)}{x_{j}} \mathrm{~d} x_{j}+\sum_{j=p_{1}+1}^{n+1} \widehat{P}_{j}(x) \mathrm{d} x_{j}
$$

\footnotetext{
12 In this way, $\mathbb{D}\left(u^{c}\right)=\mathbb{D}_{1}^{\times p_{1}} \times \cdots \times \mathbb{D}_{s}^{\times p_{s}}$, where $\mathbb{D}_{\alpha}=\left\{x \in \mathbb{C}|| x-\lambda_{\alpha} \mid \leq \epsilon_{0}\right\}, \alpha=1, \ldots, s$. 
where

$$
\begin{aligned}
& \frac{P_{j}(x)}{x_{j}}=\frac{B_{j}(x)}{x_{j}}-\omega_{j}(x), \quad 1 \leq j \leq p_{1}, \\
& \widehat{P}_{j}(x)=\frac{B_{j}(x)}{x_{j}-x_{n+1}}+\omega_{j}(x), \quad p_{1}+1 \leq j \leq n, \\
& \widehat{P}_{n+1}(x)=\sum_{j=p_{1}+1}^{n} \frac{B_{j}(x)}{x_{n+1}-x_{j}}+\sum_{j=1}^{p_{1}} \omega_{j}(x)
\end{aligned}
$$

The Pfaffian system is assumed integrable with holomorphic in $\mathbb{D}\left(u^{c}\right)$ coefficients, therefore $P_{1}(x), \ldots, P_{p_{1}}(x)$ and $\widehat{P}_{p_{1}+1}(x), \ldots, \widehat{P}_{n+1}(x)$ are holomorphic at $(\underbrace{0, \ldots, 0}_{p_{1}}, \boldsymbol{x}^{\prime}, 0)$, for $\boldsymbol{x}^{\prime}$ varying as $u_{p_{1}+1}, \ldots, u_{n}$ vary in $\mathbb{D}\left(u^{c}\right)$.

Remark 6.1 The commutation relations (4.1) at $u=(\underbrace{\lambda_{1}, \ldots, \lambda_{1}}_{p_{1}}, \boldsymbol{u}^{\prime})$, where $\boldsymbol{u}^{\prime}:=$ $\left(u_{p_{1}+1}, \ldots, u_{n}\right)$, are

$$
\left[B_{i}\left(\lambda_{1}, \ldots, \lambda_{1}, \boldsymbol{u}^{\prime}\right), B_{j}\left(\lambda_{1}, \ldots, \lambda_{1}, \boldsymbol{u}^{\prime}\right)\right]=0, \quad 1 \leq i \neq j \leq p_{1}
$$

They also follow from the integrability condition $d P(x)=P(x) \wedge P(x)$ of (6.3), which implies

$$
\frac{\partial}{\partial x_{i}}\left(\frac{P_{j}}{x_{j}}\right)-\frac{\partial}{\partial x_{j}}\left(\frac{P_{i}}{x_{i}}\right)-\frac{P_{i} P_{j}-P_{j} P_{i}}{x_{i} x_{j}}=0, \quad 1 \leq i \neq j \leq p_{1} .
$$

Let $\hat{\boldsymbol{k}}=\left(k_{1}, \ldots, k_{p_{1}}\right)$, and write $\hat{\boldsymbol{l}} \leq \hat{\boldsymbol{k}}$ if $k_{i} \leq l_{i}$ for all $i \in\left\{1, \ldots, p_{1}\right\}$. The Taylor convergent series $P_{i}(x)=\sum_{k_{1}+\cdots+k_{p_{1}} \geq 0} P_{i, \hat{\boldsymbol{k}}}\left(\boldsymbol{x}^{\prime}, x_{n+1}\right) x_{1}^{k_{1}} \cdots x_{p_{1}}^{k_{p_{1}}}$, has coefficients $P_{i, \widehat{k}}\left(\boldsymbol{x}^{\prime}, x_{n+1}\right)$ holomorphic of $\boldsymbol{x}^{\prime}, x_{n+1}$. The integrability condition becomes [63]

$$
k_{j} P_{i, \hat{\boldsymbol{k}}}-k_{i} P_{j, \hat{\boldsymbol{k}}}+\sum_{\mathbf{0} \leq \hat{\boldsymbol{l}} \leq \hat{\boldsymbol{k}}}\left[P_{i, \hat{l}}, P_{j, \hat{\boldsymbol{k}}-\hat{l}}\right]=0, \quad 1 \leq i \neq j \leq p_{1} .
$$

In particular, $P_{i, \hat{\mathbf{0}}}\left(\boldsymbol{x}^{\prime}, x_{n+1}\right)=B_{i}(\underbrace{\lambda_{1}, \ldots, \lambda_{1}}_{p_{1}}, \boldsymbol{u}^{\prime})$ for $\hat{\boldsymbol{k}}=\hat{\mathbf{0}}$, so that (6.5) reduces to (6.4).

Let us define Jordan matrices

$$
\widehat{T}^{(j)}=\operatorname{diag}(0, \ldots, 0, \underbrace{-1-\lambda_{j}^{\prime}}_{\text {position } j}, 0, \ldots, 0), \quad \text { for } \lambda_{j}^{\prime} \neq-1
$$




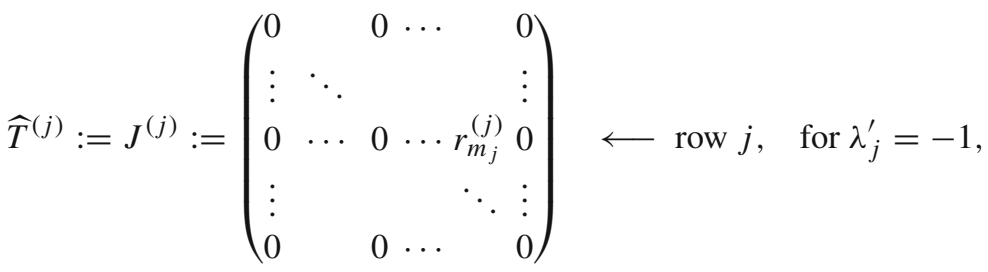

$r_{m_{j}}^{(j)}:=1, \quad$ is the only non-zero entry in position $\left(j, m_{j}\right)$, with $m_{j} \geq p_{1}+1$.

Lemma 6.1 Under the assumptions of Theorem 5.1, for every $j \in\{1, \ldots, n\}$ there exists a holomorphically invertible matrix $G^{(j)}(u)$ on $\mathbb{D}\left(u^{c}\right)$ reducing $B_{j}(u)$ to constant Jordan form. Moreover, $B_{1}\left(u^{c}\right), \ldots, B_{p_{1}}\left(u^{c}\right)$ are simultaneously reducible to $\widehat{T}^{(1)}, \ldots, \widehat{T}^{\left(p_{1}\right)}$, respectively.

Proof For every $j \in\{1, \ldots, n\}$, the Schlesinger system (3.3)-(3.5) implies the Frobenius integrability (on $\mathbb{D}\left(u^{c}\right)$ ) of the the linear Pfaffian system (see Corollary 9.1, Appendix A)

$$
\frac{\partial G^{(j)}}{\partial u_{k}}=\left(\frac{B_{k}}{u_{k}-u_{j}}+\gamma_{k}\right) G^{(j)}, \quad k \neq j, \quad \frac{\partial G^{(j)}}{\partial u_{j}}=-\sum_{k \neq j}\left(\frac{B_{k}}{u_{k}-u_{j}}+\gamma_{k}\right) G^{(j)}
$$

From (3.4)-(3.5) and the above, we receive $\partial_{k}\left(\left(G^{(j)}\right)^{-1} B_{j} G^{(j)}\right)=0, k=1, \ldots, n$, for a holomorphic on $\mathbb{D}\left(u^{c}\right)$ fundamental matrix solution $G^{(j)}(u)$. Thus, up to $G^{(j)} \mapsto$ $G^{(j)} \mathcal{G}^{(j)}, \mathcal{G}^{(j)} \in G L(n, \mathbb{C})$, we can choose $G^{(j)}(u)$ which puts $B_{j}$ in constant Jordan form. If we consider each $B_{j}$ separately, now for $j \in\left\{1, \ldots, p_{1}\right\}$, it is straightforward that the Jordan forms are the matrices $\widehat{T}^{(j)} .{ }^{13}$ An elementary computation shows that $B_{1}\left(u^{c}\right), \ldots, B_{p_{1}}\left(u^{c}\right)$ are actually reducible to $\widehat{T}^{(1)}, \ldots, \widehat{T}^{(1)}$ simultaneously, ${ }^{14}$ because only the $j$ th row of $B_{j}\left(u^{c}\right)$ is non-zero, and by (4.1) the first $p_{1}$ entries of this

13 It is also elementary to find a holomorphic $G^{(j)}$ explicitly. For example, if all $B_{j}(u)$ are diagonalizable (i.e $\left.\lambda_{j}^{\prime} \neq-1\right)$, an elementary computation shows that $\left(G^{(j)}(u)\right)^{-1} B_{j}(u) G^{(j)}(u)=\operatorname{diag}(0, \ldots, 0,-1-$ $\left.\lambda_{j}^{\prime}, 0, \ldots, 0\right), j=1,2, \ldots, n$, where the columns of $G^{(j)}$ are as follows:

$j$ th column is multiple of $\vec{e}_{j} \in \mathbb{C}^{n} ; \quad l$ th column, $l \neq j$, is multiple of $\vec{e}_{l}-\frac{A_{j l}(u)}{\lambda_{j}^{\prime}+1} \vec{e}_{j}$.

\footnotetext{
14 For example, in case of the previous footnote, the simultaneous reduction to Jordan form at $u^{*}=$ $\left(\lambda_{1}, \ldots, \lambda_{1}, \boldsymbol{u}^{\prime}\right)$, where $\left.\boldsymbol{u}^{\prime}=\left(u_{p_{1}+1}, \ldots, u_{n}\right)\right)$, is realized by the product $G^{(1)}\left(u^{*}\right) \cdots G^{\left(p_{1}\right)}\left(u^{*}\right)$, which depends holomorphically on $\boldsymbol{u}^{\prime}$
} 
row are zero, except for the $(j, j)$-entry equal to $-\lambda_{j}^{\prime}-1$. Namely,

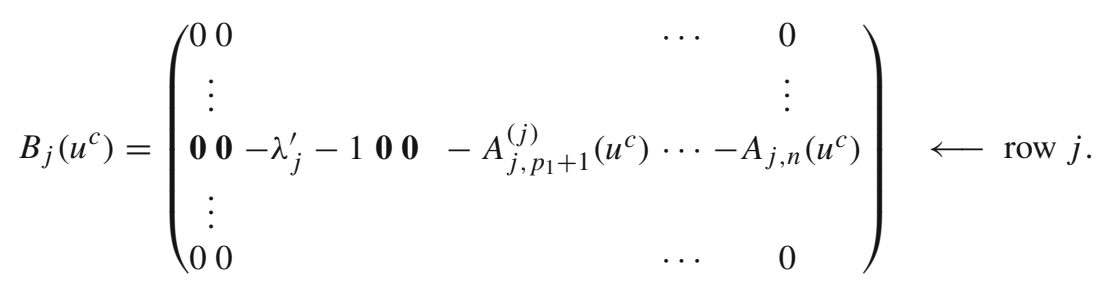

Remark 6.2 As in Lemma $6.1, B_{1}\left(u^{c}\right), \ldots, B_{p_{1}}\left(u^{c}\right)$ are reducible simultaneously to their respective Jordan forms, $B_{p_{1}+1}\left(u^{c}\right), \ldots, B_{p_{1}+p_{2}}\left(u^{c}\right)$ are reducible simultaneously to their respective Jordan forms, and so on up to $B_{p_{1}+\cdots+p_{s-1}+1}\left(u^{c}\right), \ldots, B_{p_{1}+}$ $\cdots+p_{s}\left(u^{c}\right)$.

For short, let $\boldsymbol{p}_{1}:=\left(1, \ldots, p_{1}\right)$. Without loss of generality, we label $u_{1}, \ldots, u_{p_{1}}$ so that

$$
\lambda_{j}^{\prime} \in \mathbb{C} \backslash \mathbb{Z}, \quad \text { for } 1 \leq j \leq q_{1}, \quad \lambda_{j}^{\prime} \in \mathbb{Z}, \quad \text { for } q_{1}+1 \leq j \leq p_{1} .
$$

If all $\lambda_{j}^{\prime} \in \mathbb{Z}$, then $q_{1}=0$, if all $\lambda_{j}^{\prime} \notin \mathbb{Z}$, then $q_{1}=p_{1}$. The first and fundamental step to achieve Theorem 5.1 is the following

Theorem 6.1 In the assumptions of Theorem 5.1, the Pfaffian system (5.3) admits the fundamental matrix solution

$$
\begin{aligned}
& \Psi^{\left(\boldsymbol{p}_{1}\right)}(\lambda, u)=G^{\left(\boldsymbol{p}_{1}\right)} U^{\left(\boldsymbol{p}_{1}\right)}(\lambda, u) \cdot \prod_{l=1}^{p_{1}}\left(\lambda-u_{l}\right)^{\widehat{T}^{(l)}} \cdot \prod_{j=q_{1}+1}^{p_{1}}\left(\lambda-u_{j}\right)^{\widehat{R}^{(j)}}, \\
& (\lambda, u) \in \mathcal{P}(u) \hat{\times} \mathbb{D}\left(u^{c}\right),
\end{aligned}
$$

where $G^{\left(p_{1}\right)}$ is a constant invertible matrix simultaneously reducing $B_{1}\left(u^{c}\right), \ldots$, $B_{p_{1}}\left(u^{c}\right)$ to $\widehat{T}^{(1)}, \ldots, \widehat{T}^{\left(p_{1}\right)}$ as in (6.6)-(6.7). The matrix function $U^{\left(\boldsymbol{p}_{1}\right)}(\lambda, u)$ is holomorphic in $\mathbb{D}_{1} \times \mathbb{D}\left(u^{c}\right)$ with convergent expansion

$$
\begin{aligned}
& U^{\left(\boldsymbol{p}_{1}\right)}(\lambda, u)=I+ \\
& +\sum_{\boldsymbol{k}>0, k_{1}+\cdots+k_{p_{1}} \geq 0}\left[U_{\boldsymbol{k}}^{\left(\boldsymbol{p}_{1}\right)} \cdot\left(u_{p_{1}+1}-u_{p_{1}+1}^{c}\right)^{k_{p_{1}+1}} \cdots\left(u_{n}-u_{n}^{c}\right)^{k_{n}}\left(\lambda-\lambda_{1}\right)^{k_{n+1}}\right] \\
& \quad\left(\lambda-u_{1}\right)^{k_{1}} \cdots\left(\lambda-u_{p_{1}}\right)^{k_{p_{1}}},
\end{aligned}
$$

and constant matrix coefficient $U_{\boldsymbol{k}}^{\left(\boldsymbol{p}_{1}\right)}$. Here $\boldsymbol{k}:=\left(k_{1}, \ldots, k_{n}, k_{n+1}\right), k_{j} \geq 0$, and $\boldsymbol{k}>$ 0 means that at least one $k_{j}>0(j=1, \ldots, n+1)$. The exponents $\widehat{R}^{\left(q_{1}+1\right)}, \ldots, \widehat{R}^{\left(p_{1}\right)}$ are constant nilpotent matrices. 
- If $\lambda_{j}^{\prime}=-1$,

$$
\widehat{R}^{(j)}=0 .
$$

- If $\lambda_{j}^{\prime} \in \mathbb{N}=\{0,1,2, \ldots\}$, only the entries $\widehat{R}_{m j}^{(j)}=: r_{m}^{(j)}$, for $m=1, \ldots, n$ and $m \neq j$, are possibly non zero, namely

$$
\widehat{R}^{(j)}=\left[\begin{array}{l|l|l|l|l|l|l}
\overrightarrow{0} & \cdots & \overrightarrow{0} & \sum_{m \neq j, m=1}^{n} r_{m}^{(j)} \vec{e}_{m} & \overrightarrow{0} & \cdots & \overrightarrow{0}
\end{array},\right.
$$

where only the $j$ th column is possibly non-zero.

- If $\lambda_{j}^{\prime} \in-\mathbb{N}-2=\{-2,-3, \ldots\}$, only the entries $\widehat{R}_{j m}^{(j)}=: r_{m}^{(j)}$, for $m=1, \ldots, n$ and $m \neq j$, are possibly non zero, namely

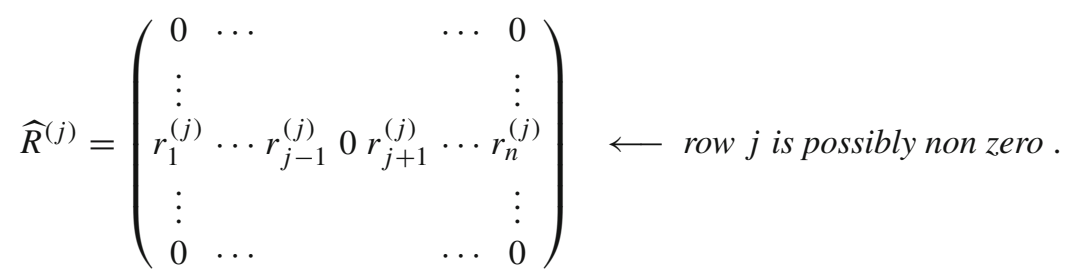

The exponents $\widehat{T}^{(l)}$ and $R^{(j)}$ satisfy the following commutation relations

$$
\begin{aligned}
& {\left[\widehat{T}^{(i)}, \widehat{T}^{(j)}\right]=0, \quad i, j=1, \ldots, p_{1} ;} \\
& {\left[\widehat{R}^{(j)}, \widehat{R}^{(k)}\right]=0, \quad\left[\widehat{T}^{(i)}, \widehat{R}^{(j)}\right]=0, \quad i=1, \ldots, p_{1}, \quad i \neq j, \quad j, k=q_{1}+1, \ldots, p_{1} .}
\end{aligned}
$$

By analytic continuation, $\Psi^{\left(p_{1}\right)}(\lambda, u)$ defines an analytic function on the universal covering of $\mathcal{P}_{\eta}(u) \hat{\times} \mathbb{D}\left(u^{c}\right)$. Another representation of (6.9) will be given in (6.24).

Proof We apply the results of [63] at the point $x=x^{c}:=(\underbrace{0,0, \ldots, 0}_{p_{1}}, \boldsymbol{x}_{c}^{\prime}, 0)$, with $\boldsymbol{x}_{c}^{\prime}:=\left(x_{p_{1}+1}^{c}, \ldots, x_{n}^{c}\right)$, corresponding to $u=u^{c}$ and $\lambda=\lambda_{1}$, where $x_{j}^{c}=$ $u_{j}^{c}-\lambda_{1}, j=p_{1}+1, \ldots, n$. By Theorem 7 of [63], the Pfaffian system (6.3) admits a fundamental matrix solution

$$
\Psi^{\left(p_{1}\right)}(\lambda, u)=U_{0} U(x) Z(x), \quad Z(x)=\prod_{j=1}^{p_{1}} x_{l}^{A_{j}} \prod_{j=1}^{p_{1}} x_{l}^{Q_{j}}, \quad \operatorname{det} U_{0} \neq 0,
$$

for certain matrices $A_{j}$ which are simultaneous triangular forms of $B_{1}\left(u^{c}\right), \ldots, B_{p_{1}}\left(u^{c}\right)$. While in [63] a lower triangular form is considered, we equivalently use the upper triangular one. The matrices $Q_{j}$ will be described below. The matrix $U(x)=V(x) \cdot W(x)$ has structure

$$
V(x)=I+\sum_{\boldsymbol{k}>0, k_{p_{1}+1}+\cdots+k_{n+1}>0} V_{\boldsymbol{k}} x_{1}^{k_{1}} \cdots x_{p_{1}}^{k_{p_{1}}}\left(x_{p_{1}+1}-x_{p_{1}+1}^{c}\right)^{k_{p_{1}+1}}
$$




$$
\begin{aligned}
& \cdots\left(x_{n}-x_{n}^{c}\right)^{k_{n}} \cdot x_{n+1}^{k_{n+1}} \\
& W(x)=I+\sum_{k_{1}+\cdots+k_{p_{1}}>0} W_{k_{1}, \ldots, k_{p_{1}}} x_{1}^{k_{1}} \cdots x_{p_{1}}^{k_{p_{1}}} .
\end{aligned}
$$

The constant matrix coefficients $V_{k}, W_{k_{1}, \ldots, k_{p_{1}}}$ can be determined [63] from the constant matrix coefficients $P_{i, k}$ in the Taylor expansion ${ }^{15}$ of the $P_{j}(x)$ and $\widehat{P}_{j}(x)$. Recall that $x_{j}=\lambda-u_{j}, 1 \leq j \leq p_{1}$, and $x_{n+1}=\lambda-\lambda_{1}$. Moreover, for $p_{1}+1 \leq j \leq n$, we have $x_{j}-x_{j}^{c}=\left(u_{j}-\lambda_{1}\right)-\left(u_{j}^{c}-\lambda_{1}\right)=u_{j}-u_{j}^{c}$. Thus, restoring variables $(\lambda, u)$, we have

$$
\begin{aligned}
& V(\lambda, u)=I+ \\
& +\sum_{k_{p_{1}+1}+\cdots+k_{n+1}>0}\left[V_{k}\left(u_{p_{1}+1}-u_{p_{1}+1}^{c}\right)^{k_{p_{1}+1}} \cdot \ldots \cdot\left(u_{n}-u_{n}^{c}\right)^{k_{n}} \cdot\left(\lambda-\lambda_{1}\right)^{k_{n+1}}\right] \\
& \left(\lambda-u_{1}\right)^{k_{1}} \cdots\left(\lambda-u_{p_{1}}\right)^{k_{p_{1}}}, \\
& W\left(\lambda, u_{1}, \ldots, u_{p_{1}}\right)=I+\sum_{k_{1}+\cdots+k_{p_{1}}>0} W_{k_{1}, \ldots, k_{p_{1}}}\left(\lambda-u_{1}\right)^{k_{1}} \cdot \ldots \cdot\left(\lambda-u_{p_{1}}\right)^{k_{p_{1}}} .
\end{aligned}
$$

Therefore, the matrices appearing in the statement are $G^{\left(p_{1}\right)}:=U_{0}$ and $U^{\left(p_{1}\right)}(\lambda, u):=$ $V(\lambda, u) W(\lambda, u)$, which is holomorphic for $(\lambda, u) \in \mathbb{D}_{1} \times \mathbb{D}\left(u^{c}\right)$.

We show that the exponents $A_{j}$ and $Q_{j}$ are, respectively, $\widehat{T}^{(j)}$ in (6.6)-(6.7) and $\widehat{R}^{(j)}$ in (6.10)-(6.11)-(6.12). According to [63] (see theorems 2 and 5), the matrix function $G^{\left(\boldsymbol{p}_{1}\right)} \cdot U^{\left(\boldsymbol{p}_{1}\right)}(\lambda, u)$ in (6.9) provides the gauge transformation

$$
\Psi=G^{\left(p_{1}\right)} \cdot U^{\left(p_{1}\right)}(\lambda, u) Z \underset{\text { in notation of [63] }}{\equiv} U_{0} U(x) Z,
$$

which brings (6.3) to the reduced form (being "reduced" is defined in [63])

$$
d Z=\sum_{j=1}^{p_{1}} \frac{Q_{j}(x)}{x_{j}} Z, \quad Q_{j}(x)=A_{j}+\sum_{\widehat{\boldsymbol{k}}>0} Q_{\widehat{\boldsymbol{k}}, j} x_{1}^{k_{1}} \cdots x_{p_{1}}^{k_{p_{1}}}
$$

where the notation $\widehat{\boldsymbol{k}}=\left(k_{1}, \ldots, k_{p_{1}}\right)>0$ means at least one $k_{l}>0$. From [63], we have the following.

- The $A_{j}$ are simultaneous triangular forms of $B_{1}\left(u^{c}\right), \ldots, B_{p_{1}}\left(u^{c}\right)$. Thus, by Lemma 6.1, they can be taken to be

$$
A_{j}=\widehat{T}^{(j)} \text { as in (6.6) - (6.7), } j=1, \ldots, p_{1} .
$$

15

$$
P_{i}(x)=\sum_{k_{1}+\cdots+k_{n+1} \geq 0} P_{i, \boldsymbol{k}} x_{1}^{k_{1}} \cdots x_{p_{1}}^{k_{p_{1}}} \cdot\left(x_{p_{1}+1}-x_{p_{1}+1}^{c}\right)^{k_{p_{1}+1}} \cdots\left(x_{n}-x_{n}^{c}\right)^{k_{n}} \cdot x_{n+1}^{k_{n+1}} .
$$

and analogous for $\widehat{P}_{j}(x)$ 
- The $Q_{\widehat{k}, j} \operatorname{satisfy} \operatorname{diag}\left(Q_{\widehat{k}, j}\right)=0$, while the entry $(\alpha, \beta)$ for $\alpha \neq \beta$ satisfies

$$
\left(Q_{\widehat{\boldsymbol{k}}, j}\right)_{\alpha \beta} \neq 0 \quad \text { only if } \quad\left(\widehat{T}^{(j)}\right)_{\alpha \alpha}-\left(\widehat{T}^{(j)}\right)_{\beta \beta}=k_{j} \geq 0, \quad \text { for all } j=1, \ldots, p_{1} \text {. }
$$

Taking into account the particular structure (6.6)-(6.7), the above condition can be satisfied only for

$$
\widehat{\boldsymbol{k}}=(\underbrace{0, \ldots, 0}_{q_{1}}, \underbrace{0, \ldots, 0, k_{j}, 0, \ldots, 0}_{p_{1}-q_{1}}), \quad k_{j}=\left|\lambda_{j}^{\prime}+1\right| \geq 1 \text { in position } j,
$$

because

$$
\begin{aligned}
& \left(\widehat{T}^{(j)}\right)_{\alpha \alpha}-\left(\widehat{T}^{(j)}\right)_{\beta \beta}=-\lambda_{j}^{\prime}-1 \geq 1 \quad \text { when } \lambda_{j}^{\prime} \in-\mathbb{N}-2 \quad \text { and } \alpha=j \quad(\beta \neq j), \\
& \left(\widehat{T}^{(j)}\right)_{\alpha \alpha}-\left(\widehat{T}^{(j)}\right)_{\beta \beta}=\lambda_{j}^{\prime}+1 \geq 1 \quad \text { when } \lambda_{j}^{\prime} \in \mathbb{N} \quad \text { and } \beta=j \quad(\alpha \neq j) .
\end{aligned}
$$

This can occur only for $j=q_{1}+1, \ldots, p_{1}$. Thus

$$
Q_{\widehat{\boldsymbol{k}}, j}=0, \quad j=1, \ldots, q_{1}, \quad Q_{\widehat{\boldsymbol{k}}, j}=\widehat{R}^{(j)} \text { in }(6.10)-(6.11)-(6.12), \quad j=q_{1}+1, \ldots, p_{1} .
$$

In conclusion, the reduced form turns out to be

$$
d Z=\left[\sum_{j=1}^{p_{1}}\left(\frac{\widehat{T}^{(j)}+\widehat{R}^{(j)} x^{k_{j}}}{x_{j}}\right)\right] Z, \quad \widehat{R}^{(1)}=\cdots=\widehat{R}^{\left(q_{1}\right)}=0 .
$$

Its integrability implies the commutation relations. Indeed, the compatibility $\partial_{i} \partial_{j} Z=$ $\partial_{j} \partial_{i} Z, i \neq j$, holds if and only if

$$
\begin{aligned}
& \frac{\left[\widehat{T}^{(j)}, \widehat{T}^{(i)}\right]}{x_{i} x_{j}}+\left[\widehat{R}^{(j)}, \widehat{R}^{(i)}\right] x_{i}^{k_{i}-1} x_{j}^{k_{j}-1}+\left[\widehat{T}^{(j)}, \widehat{R}^{(i)}\right] x_{i}^{k_{i}-2} \\
& \quad+\left[\widehat{R}^{(j)}, \widehat{T}^{(i)}\right] x_{j}^{k_{j}-2}=0, \quad 1 \leq i \neq j \leq p_{1} .
\end{aligned}
$$

Keeping into account that $\widehat{R}^{(1)}=\cdots=\widehat{R}^{\left(q_{1}\right)}=0$, the above holds if and only if (6.13)-(6.14) hold.

The last to be checked is that a fundamental matrix of (6.19) is $Z(x)$ in (6.15), namely

$$
Z(x)=\prod_{l=1}^{p_{1}} x_{l}^{\widehat{T}^{(l)}} \prod_{j=q_{1}+1}^{p_{1}} x_{l}^{\widehat{R}^{(j)}}
$$

It suffices to verify this by differentiating $Z(x)$, keeping into account the commutation relations (6.13)-(6.14) and the formula $\partial_{i} x_{i}^{M}=\left(M / x_{i}\right) x_{i}^{M}$, for a constant matrix $M$. 
For $i=1, \ldots, q_{1}$, we receive

$$
\frac{\partial}{\partial x_{i}} Z(x)=\frac{\widehat{T}^{(i)}}{x_{i}} Z(x)
$$

For $i=q_{1}+1, \ldots, p_{1}$, we receive

$$
\begin{aligned}
\frac{\partial}{\partial x_{i}} Z(x) & =\frac{T^{(i)}}{x_{i}} Z(x)+\left(\prod_{l=1}^{p_{1}} x_{l}^{\widehat{T}^{(l)}}\right) \frac{\widehat{R}^{(i)}}{x_{i}}\left(\prod_{j=q_{1}+1}^{p_{1}} x_{l}^{\widehat{R}^{(j)}}\right) \\
& =\frac{\widehat{T}^{(i)}}{x_{i}} Z(x)+\left(\prod_{l=1}^{i-1} x_{l}^{\widehat{T}^{(l)}}\right) \frac{x_{i}^{\widehat{T}^{(i)}} \widehat{R}^{(i)}}{x_{i}}\left(\prod_{l=i+1}^{p_{1}} x_{l}^{\widehat{T}^{(l)}}\right)\left(\prod_{j=q_{1}+1}^{p_{1}} x_{l}^{\widehat{R}^{(j)}}\right)=(* *) .
\end{aligned}
$$

Now, recalling that $k_{i}=\left|\lambda_{i}^{\prime}+1\right|$ and (6.16)-(6.17), we see that $x_{i}^{\widehat{T}^{(i)}} \widehat{R}^{(i)} x_{i}^{-\widehat{T}^{(i)}}=$ $\widehat{R}^{(i)} x_{i}^{k_{i}}$. Therefore,

$$
(* *)=\frac{\widehat{T}^{(i)}}{x_{i}} Z(x)+\frac{\widehat{R}^{(i)} x_{i}^{k_{i}}}{x_{i}}\left(\prod_{l=1}^{p_{1}} x_{l}^{\widehat{T}^{(l)}}\right)\left(\prod_{j=q_{1}+1}^{p_{1}} x_{l}^{\widehat{R}^{(j)}}\right)=\frac{\widehat{T}^{(i)}+\widehat{R}^{(i)} x_{i}^{k_{i}}}{x_{i}} Z(x),
$$

as we wanted to prove.

Finally, the fact that $\Psi^{\left(p_{1}\right)}(\lambda, u)$ has analytic continuation on the universal covering of $\mathcal{P}_{\eta}(u) \hat{\times} \mathbb{D}\left(u^{c}\right)$ follows from general results in the theory of linear Pfaffian systems $[28,32,63]$.

It is convenient to introduce a slight change of the exponents. Without loss in generality, we can label $u_{1}, \ldots, u_{p_{1}}$ in such a way that, for some $q_{1}, c_{1} \geq 0$ integers, the following ordering of eigenvalues of $A$ holds:

$\underline{\lambda_{1}^{\prime}, \ldots, \lambda_{q_{1}}^{\prime} \in \mathbb{C} \backslash \mathbb{Z}, \quad \lambda_{q_{1}+1}^{\prime}, \ldots, \lambda_{q_{1}+c_{1}}^{\prime} \in \mathbb{Z}_{-}, \quad \lambda_{q_{1}+c_{1}+1}^{\prime}, \ldots, \lambda_{p_{1}}^{\prime} \in \mathbb{N}}$

Clearly, $0 \leq q_{1} \leq p_{1}, 0 \leq c_{1} \leq p_{1}$ and $0 \leq q_{1}+c_{1} \leq p_{1}$. We define new exponents.

- For $\lambda_{j}^{\prime} \neq-1$,

$$
T^{(j)}:=\widehat{T}^{(j)}, \quad j=1, \ldots, p_{1} ; \quad R^{(j)}:=\widehat{R}^{(j)}, \quad j=q_{1}+1, \ldots, p_{1} .
$$

- For $\lambda_{j}^{\prime}=-1\left(\right.$ so $\left.j \in\left\{q_{1}+1, \ldots, q_{1}+c_{1}\right\}\right)$

$$
T^{(j)}:=0, \quad R^{(j)}:=\underbrace{J^{(j)}}_{\operatorname{in}(6.7)}=\left(\begin{array}{cccccc}
0 & & 0 & \cdots & & 0 \\
\vdots & \ddots & & & \vdots \\
0 & \cdots & 0 & \cdots & r_{m_{j}}^{(j)} & 0 \\
\vdots & & & & \ddots & \vdots \\
0 & & 0 & \cdots & & 0
\end{array}\right) \longleftarrow \text { row } j, \quad r_{m_{j}}^{(j)}=1
$$


Recall that $m_{j} \geq p_{1}+1$.

This new definitions allow to treat together the case $\lambda_{j}^{\prime} \in-\mathbb{N}-2$ and the case $\lambda_{j}^{\prime}=-1$.

Lemma 6.2 With the definition (6.20)-(6.21), the following relations hold.

$$
\begin{aligned}
& {\left[T^{(i)}, T^{(j)}\right]=0, \quad i, j=1, \ldots, p_{1} ;} \\
& {\left[R^{(j)}, R^{(k)}\right]=0, \quad\left[T^{(i)}, R^{(j)}\right]=0, \quad i=1, \ldots, p_{1}, \quad i \neq j, \quad j, k=q_{1}+1, \ldots, p_{1},}
\end{aligned}
$$

Proof The equivalence between (6.13)-(6.14) and (6.22)-(6.23) is straightforward.

Corollary 6.1 In Theorem 6.1, the fundamental matrix solution (6.9) is

$$
\Psi^{\left(\boldsymbol{p}_{1}\right)}(\lambda, u)=G^{\left(\boldsymbol{p}_{1}\right)} \cdot U^{\left(\boldsymbol{p}_{1}\right)}(\lambda, u) \cdot \prod_{l=1}^{p_{1}}\left(\lambda-u_{l}\right)^{T^{(l)}} \cdot \prod_{j=q_{1}+1}^{p_{1}}\left(\lambda-u_{j}\right)^{R^{(j)}}
$$

where the exponents are defined in (6.20)-(6.21).

Proof It is an immediate consequence of the commutation relations being satisfied, that the representation (6.9) for $\Psi^{\left(\boldsymbol{p}_{1}\right)}$ still holds with the definition (6.20)-(6.21).

The commutation relations impose a simplification on the structure of the matrices $R^{(j)}$. Let the new convention (6.20)-(6.21) be used. The relations $\left[T^{(i)}, R^{(j)}\right]=0$ for $i=1, \ldots, p_{1}$ and $j=q_{1}+1, \ldots, p_{1}, j \neq i$, imply the vanishing of the first $p_{1}$ non-trivial entries of $R^{(j)}$, so that (by (6.11), (6.12) and (6.21)),

$$
\begin{aligned}
& R^{(j)}=\left[\begin{array}{l|l|l|l|l|l|l}
\overrightarrow{0} & \cdots & \overrightarrow{0} & \sum_{m=p_{1}+1}^{n} r_{m}^{(j)} \vec{e}_{m} & \overrightarrow{0} & \cdots & \overrightarrow{0}
\end{array}, \quad \lambda_{j}^{\prime} \in \mathbb{N} .\right.
\end{aligned}
$$

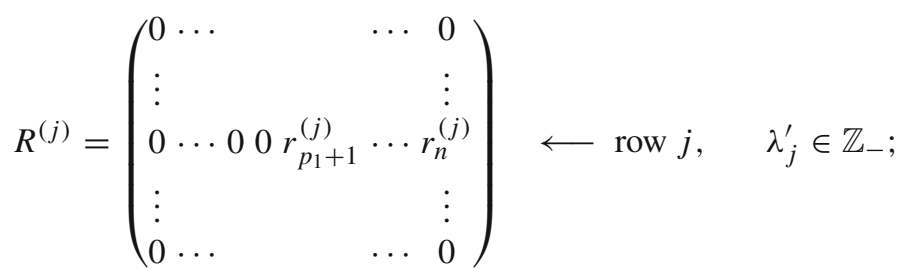

The relations $\left[R^{(j)}, R^{(k)}\right]=0$ for either $j, k \in\left\{q_{1}+1, \ldots, q_{1}+c_{1}\right\}$ or $j, k \in$ $\left\{q_{1}+c_{1}+1, \ldots, p_{1}\right\}$ are automatically satisfied. On the other hand, the commutators $\left[R^{(j)}, R^{(k)}\right]=0$ for $j \in\left\{q_{1}+1, \ldots, q_{1}+c_{1}\right\}$ and $k \in\left\{q_{1}+c_{1}+1, \ldots, p_{1}\right\}$ imply the further (quadratic) relations

$$
\sum_{m=p_{1}+1}^{n} r_{m}^{(j)} r_{m}^{(k)}=0
$$

In particular, if $\lambda_{j}^{\prime}=-1$ and $R^{(j)}$ is (6.21), all the above conditions can be satisfied, provided that we take $m_{j} \geq p_{1}+1$, as we have agreed from the beginning. 


\subsection{Selected vector solutions $\vec{\Psi}_{i}$}

Remark on notations For the sake of the proof, it is convenient to use a slightly different notation with respect to the statement of Theorem 5.1. The identifications between objects in the proof and objects in the statement is $\vec{\varphi}_{i} \longmapsto \vec{\psi}_{i}, r_{i}^{(m)} / r_{k}^{(i)} \longmapsto$ $r_{m}$ and $\vec{\varphi}_{k} / r_{k}^{(i)} \longmapsto \phi_{i}$.

We will construct selected vector solutions of Theorem 5.1 from suitable linear combinations of columns of the fundamental matrix $\Psi^{\left(p_{1}\right)}$ in (6.24). The $i$ th column of an $n \times n$ matrix $M$ is $M \cdot \vec{e}_{i}$ (rows by columns multiplication), where $\vec{e}_{i}$ is the standard unit basic vector in $\mathbb{C}^{n}$. From (6.22)-(6.23), and (6.26)-(6.25)-(6.27), we receive

$$
\begin{aligned}
& \prod_{l=1}^{p_{1}}\left(\lambda-u_{l}\right)^{T^{(l)}} \cdot \prod_{j=q_{1}+1}^{p_{1}}\left(\lambda-u_{j}\right)^{R^{(j)}} \cdot \vec{e}_{i} \\
& \quad=\left\{\begin{array}{cc}
\left(\lambda-u_{i}\right)^{-\lambda_{i}^{\prime}-1} \vec{e}_{i}, & i=1, \ldots, q_{1}+c_{1}, \quad \lambda_{i}^{\prime} \in \mathbb{C} \backslash \mathbb{N} ; \\
\left(\lambda-u_{i}\right)^{-\lambda_{i}^{\prime}-1} \vec{e}_{i}+\left(\sum_{m=p_{1}+1}^{n} r_{m}^{(i)} \vec{e}_{m}\right) \ln \left(\lambda-u_{i}\right), & i=q_{1}+c_{1}+1, \ldots, p_{1}, \quad \lambda_{i}^{\prime} \in \mathbb{N} ; \\
\vec{e}_{i}+\sum_{m=q_{1}+1}^{q_{1}+c_{1}} \vec{e}_{m} r_{i}^{(m)}\left(\lambda-u_{m}\right)^{-\lambda_{m}^{\prime}-1} \ln \left(\lambda-u_{m}\right), & i=p_{1}+1, \ldots, n .
\end{array}\right.
\end{aligned}
$$

For $i=1, \ldots, n$, let

$$
\vec{\varphi}_{i}(\lambda, u):=G^{\left(p_{1}\right)} U(\lambda, u) \cdot \vec{e}_{i}, \quad i=1, \ldots, n
$$

which is holomorphic for $(\lambda, u) \in \mathbb{D}_{1} \times \mathbb{D}\left(u^{c}\right)$. For $i=1, \ldots, p_{1}$, we define vector valued functions

$$
\vec{\Psi}_{i}(\lambda, u):=\left\{\begin{array}{l}
\vec{\varphi}_{i}(\lambda, u)\left(\lambda-u_{i}\right)^{-\lambda_{i}^{\prime}-1}, \quad i=1, \ldots, q_{1}+c_{1}, \quad \lambda_{i}^{\prime} \in \mathbb{C} \backslash \mathbb{N} \\
\sum_{k=p_{1}+1}^{n} r_{k}^{(i)} \vec{\varphi}_{k}(\lambda, u), i=q_{1}+c_{1}+1, \ldots, p_{1}, \quad \lambda_{i}^{\prime} \in \mathbb{N}
\end{array}\right.
$$

Notice that for $i=q_{1}+c_{1}+1, \ldots, p_{1}$, if $r_{k}^{(i)}=0$ for all $k=p_{1}+1, \ldots, n$, then $\vec{\Psi}_{i}(\lambda, u)$ is identically zero

$$
\vec{\Psi}_{i}(\lambda, u) \equiv 0, \quad \lambda_{i}^{\prime} \in \mathbb{N}
$$

Hence, the $i$ th column of $\Psi^{\left(p_{1}\right)}(\lambda, u)$ is

$$
\begin{array}{rlrl}
\Psi^{\left(\boldsymbol{p}_{1}\right)}(\lambda, u) \cdot \vec{e}_{i} & =\vec{\Psi}_{i}(\lambda, u), & & i=1, \ldots, q_{1}+c_{1}, \\
& =\vec{\Psi}_{i}(\lambda, u) \ln \left(\lambda-u_{i}\right)+\frac{\vec{\varphi}_{i}(\lambda, u)}{\left(\lambda-u_{i}\right)^{\lambda_{i}^{\prime}+1}}, & & i=q_{1}+c_{1}+1, \ldots, p_{1}, \\
& =\varphi_{i}(\lambda, u)+\sum_{m=q_{1}+1}^{q_{1}+c_{1}} r_{i}^{(m)} \vec{\Psi}_{m}(\lambda, u) \ln \left(\lambda-u_{m}\right), & i=p_{1}+1, \ldots, n .
\end{array}
$$


Proposition 6.1 The vector functions (6.30) coincide with the following linear combinations of columns of $\Psi^{\left(\boldsymbol{p}_{1}\right)}(\lambda, u)$,

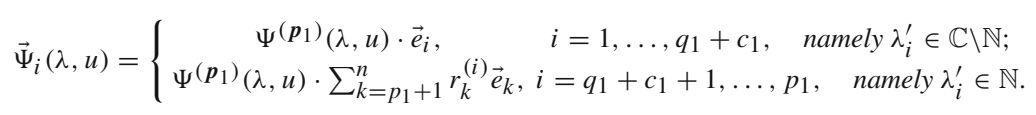

As such, they are vector solutions (called selected) of the Pfaffian system (5.3). Those $\vec{\Psi}_{i}(\lambda, u)$ which are not identically zero are linearly independent.

Proof For $i=1, \ldots, q_{1}+c_{1},(6.35)$ is just (6.32), so it is a vector solution of (5.3). In case $i=q_{1}+c_{1}+1, \ldots, p_{1}$, we claim that $\vec{\Psi}_{i}(\lambda, u)$ defined in (6.30) coincides with the following linear combination

$$
\vec{\Psi}_{i}(\lambda, u)=\sum_{k=p_{1}+1}^{n} r_{k}^{(i)}\left(\Psi^{\left(p_{1}\right)}(\lambda, u) \cdot \vec{e}_{k}\right), \quad i=q_{1}+c_{1}+1, \ldots, p_{1},
$$

of the vector solutions (6.34). Indeed,

$$
\begin{aligned}
& \sum_{k=p_{1}+1}^{n} r_{k}^{(i)}\left(\Psi^{\left(\boldsymbol{p}_{1}\right)}(\lambda, u) \cdot \vec{e}_{k}\right) \\
& =\sum_{k=p_{1}+1}^{n} r_{k}^{(i)}\left(\varphi_{k}(\lambda, u)+\sum_{m=q_{1}+1}^{q_{1}+c_{1}} r_{k}^{(m)} \vec{\Psi}_{m}(\lambda, u) \ln \left(\lambda-u_{m}\right)\right) \\
& \underset{(6.30)}{=} \vec{\Psi}_{i}(\lambda, u)+\sum_{m=q_{1}+1}^{q_{1}+c_{1}}\left(\sum_{k=p_{1}+1}^{n} r_{k}^{(i)} r_{k}^{(m)}\right) \vec{\Psi}_{m}(\lambda, u) \ln \left(\lambda-u_{m}\right) .
\end{aligned}
$$

Now, it follows from (6.27) that $\sum_{k=p_{1}+1}^{n} r_{k}^{(i)} r_{k}^{(m)}=0$, so proving the claim and the expressions (6.35). Linear independence follows from (6.35).

\subsection{Singular solutions $\vec{\Psi}_{i}^{(\operatorname{sing})}$}

Using the previous results, we define singular vector solutions of the Pfaffian system.

- For $\lambda_{i}^{\prime} \notin \mathbb{Z}$, i.e. $i=1, \ldots, q_{1}$,

$$
\vec{\Psi}_{i}^{(\text {sing })}(\lambda, u):=\vec{\Psi}_{i}(\lambda, u) \equiv \Psi^{\left(p_{1}\right)}(\lambda, u) \cdot \vec{e}_{i}
$$

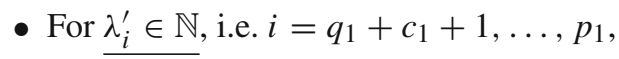

$$
\vec{\Psi}_{i}^{(\text {sing })}(\lambda, u):=\vec{\Psi}_{i}(\lambda, u) \ln \left(\lambda-u_{i}\right)+\frac{\vec{\varphi}_{i}(\lambda, u)}{\left(\lambda-u_{i}\right)^{\lambda_{i}^{\prime}+1}} \equiv \Psi^{\left(p_{1}\right)}(\lambda, u) \cdot \vec{e}_{i} .
$$


- For $\underline{\lambda}_{i}^{\prime} \in \mathbb{Z}_{-}$, i.e. $i=q_{1}+1, \ldots, q_{1}+c_{1}$, we distinguish three subcases.

i) If $\underline{\lambda_{i}^{\prime} \leq-2}$ and $r_{k}^{(i)} \neq 0$ for some $k \in\left\{p_{1}+1, \ldots, n\right\}$, from (6.34) (change notation $i \mapsto k$ )

$$
\begin{aligned}
\vec{\Psi}_{i}^{(\operatorname{sing})}(\lambda, u) & :=\frac{1}{r_{k}^{(i)}}\left\{\varphi_{k}(\lambda, u)+\sum_{m=q_{1}+1}^{q_{1}+c_{1}} r_{k}^{(m)} \vec{\Psi}_{m}(\lambda, u) \ln \left(\lambda-u_{m}\right)\right\} \\
& \equiv \frac{1}{r_{k}^{(i)}} \Psi^{\left(p_{1}\right)}(\lambda, u) \cdot \vec{e}_{k}
\end{aligned}
$$

ii) If $\underline{\lambda_{i}^{\prime} \leq-2}$ and $r_{k}^{(i)}=0$ for all $k \in\left\{p_{1}+1, \ldots, n\right\}$,

$$
\vec{\Psi}_{i}^{(\operatorname{sing})}(\lambda, u):=0
$$

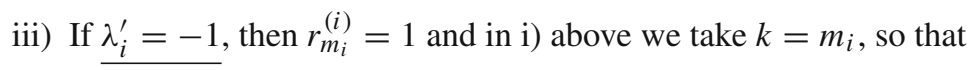

$$
\begin{aligned}
\vec{\Psi}_{i}^{(\text {sing })}(\lambda, u): & =\vec{\varphi}_{m_{i}}(\lambda, u)+\vec{\Psi}_{i}(\lambda, u) \ln \left(\lambda-u_{i}\right) \\
& +\sum_{m \neq i, m=q_{1}+1}^{q_{1}+c_{1}} r_{m_{i}}^{(m)} \vec{\Psi}_{m}(\lambda, u) \ln \left(\lambda-u_{m}\right) . \\
& =\Psi^{\left(p_{1}\right)}(\lambda, u) \cdot \vec{e}_{m_{i}}, \quad m_{i} \geq p_{1}+1 .
\end{aligned}
$$

The above $\vec{\Psi}_{i}^{(\text {sing })}(\lambda, u)$ in i) and iii) is singular at $u_{i}$, but possibly also at $u_{q_{1}+1}, \ldots, u_{q_{1}+c_{1}}$ corresponding to $\lambda_{m}^{\prime} \in \mathbb{Z}_{-}$. By definition,

$$
\vec{\Psi}_{i}^{(\text {sing })}(\lambda, u) \underset{\lambda \rightarrow u_{i}}{=} \vec{\Psi}_{i}(\lambda, u) \ln \left(\lambda-u_{i}\right)+\operatorname{reg}\left(\lambda-u_{i}\right), \quad i=q_{1}+1, \ldots, q_{1}+c_{1},
$$

Remark 6.3 The definition in (i) contains the freedom of choosing $k \in\left\{p_{1}+1, \ldots, n\right\}$, which changes $\varphi_{k}(\lambda, u)$ and the ratios $r_{k}^{(m)} / r_{k}^{(i)}$ [in formula (5.8), $\varphi_{k} / r_{k}^{(i)}$ is denoted by $\phi_{i}$ and $r_{k}^{(m)} / r_{k}^{(i)}$ is $r_{m}$ ]. Whatever is the choice of $k$, provided that $r_{k}^{(i)} \neq 0$, the behaviour at $\lambda=u_{i}$ of the corresponding $\vec{\Psi}_{i}^{(\operatorname{sing})}$ is always (6.36), so it is uniquely fixed if we fix the normalization of $\vec{\Psi}_{i}(\lambda, u)$.

As a consequence of the above definitions and Sect. 6.2, we receive the following Proposition 6.2 The $\vec{\Psi}_{i}^{(\text {sing })}(\lambda, u)$ defined above, $i=1, \ldots, p_{1}$, when not identically zero, are linearly independent. They are represented as follows

$$
\vec{\Psi}_{i}^{(\text {sing })}(\lambda, u)=\left\{\begin{array}{cc}
\Psi^{\left(p_{1}\right)}(\lambda, u) \cdot \vec{e}_{i}, & \lambda_{i}^{\prime} \in \mathbb{C} \backslash \mathbb{Z}_{-}, \\
\Psi^{\left(p_{1}\right)}(\lambda, u) \cdot \frac{\vec{e}_{k}}{r_{k}^{(i)},} & \lambda_{i}^{\prime} \in \mathbb{Z}_{-}, \quad \text { for some } \in\left\{p_{1}+1, \ldots, n\right\} \text { such that } r_{k}^{(i)} \neq 0 \\
0, & \lambda_{i}^{\prime} \in-\mathbb{N}-2, \quad \text { if } r_{k}^{(i)}=0 \text { for all } k \in\left\{p_{1}+1, \ldots, n\right\} .
\end{array}\right.
$$




\subsection{Expansions at $\lambda=u_{i}, i=1, \ldots, p_{1}$ and completion of the proof}

In order to proceed in the proof, and in view of the Laplace transform to come, we need local behaviour at $\lambda=u_{i}$.

Lemma 6.3 The following Taylor expansion holds at $\lambda=u_{i}$, with coefficients $\vec{d}_{l}^{(i)}(u)$ holomorphic on $\mathbb{D}\left(u^{c}\right)$,

$$
\vec{\Psi}_{i}(\lambda, u)=\sum_{l=0}^{\infty} \vec{d}_{l}^{(i)}(u)\left(\lambda-u_{i}\right)^{l}, \quad \lambda_{i}^{\prime} \in \mathbb{N}, \text { namely } i=q_{1}+c_{1}+1, \ldots, p_{1}
$$

Proof By (6.30), $\vec{\Psi}_{i}(\lambda, u)=G^{\left(p_{1}\right)} U(\lambda, u) \cdot\left(\sum_{m=p_{1}+1}^{n} r_{m}^{(i)} \vec{e}_{m}\right)$, so it is holomorphic on $\mathbb{D}_{1} \times \mathbb{D}\left(u^{c}\right)$. From this we conclude.

The coefficients $d_{l}^{(i)}(u)$ will be fixed by a chosen normalization for $\vec{\varphi}_{i}$ in (6.33), as in the following lemma.

Lemma 6.4 The following Taylor expansions hold at $\lambda=u_{i}$, uniformly convergent for $u \in \mathbb{D}\left(u^{c}\right)$.

$$
\begin{gathered}
\left.\lambda_{i}^{\prime} \notin \mathbb{N} \text {, i.e. } i=1, \ldots, q_{1}+c_{1}: \begin{array}{c}
\vec{\Psi}_{i}(\lambda, u) \\
\lambda_{i}^{\prime} \in \mathbb{N} \text {, i.e. } q_{1}+c_{1}+1, \ldots, p_{1}: \frac{\vec{\varphi}_{i}(\lambda, u)}{\left(\lambda-u_{i}\right)^{\lambda_{i}^{\prime}+1}}
\end{array}\right\} \\
\underset{\lambda \rightarrow u_{i}}{=}\left(f_{i} \vec{e}_{i}+\sum_{l=1}^{\infty} \vec{b}_{l}^{(i)}(u)\left(\lambda-u_{i}\right)^{l}\right)\left(\lambda-u_{i}\right)^{-\lambda_{i}^{\prime}-1},
\end{gathered}
$$

with certain vector coefficients $\vec{b}_{l}^{(i)}(u)$ holomorphic in $\mathbb{D}\left(u^{c}\right)$. In particular, the leading term is constant, and will be chosen as follows

$$
f_{i}=\left\{\begin{array}{ccc}
\Gamma\left(\lambda_{i}^{\prime}+1\right), & \lambda_{i}^{\prime} \in \mathbb{C} \backslash \mathbb{Z}, & i=1, \ldots, q_{1}, \\
\frac{(-1)^{\lambda_{i}^{\prime}}}{\left(-\lambda_{i}^{\prime}-1\right) !}, & \lambda_{i}^{\prime} \in \mathbb{Z}_{-}, \quad i=q_{1}+1, \ldots, q_{1}+c_{1}, \\
\lambda_{i}^{\prime} ! \equiv \Gamma\left(\lambda_{i}^{\prime}+1\right), & \lambda_{i}^{\prime} \in \mathbb{N}, \quad i=q_{1}+c_{1}+1, \ldots, p_{1} .
\end{array}\right.
$$

Proof That the above convergent expansions must hold follows from the definitions. Work is required to prove that the leading term is $f_{i} \vec{e}_{i}$, with $f_{i} \in \mathbb{C} \backslash\{0\}$.

From definitions (6.29)-(6.30), the leading term must coincide with the leading term of the expansion at $\lambda=u_{i}$ of the $i$ th column $G^{\left(p_{1}\right)} U(\lambda, u) \cdot \vec{e}_{i}$, for $i=1, \ldots, p_{1}$. To evaluate it, observe that the solution $\Psi^{\left(p_{1}\right)}(\lambda, u)$, restricted to a polydisc $\mathbb{D}\left(u^{0}\right)$ contained in a $\tau$-cell of $\mathbb{D}\left(u^{c}\right)$, is a fundamental matrix solution of the Fuchsian system (1.4) in the Levelt form (6.38) at $\lambda=u_{i}, i=1, \ldots, p_{1}$. Indeed, by (6.23) it 
can be written as

$$
\begin{aligned}
& \Psi^{\left(\boldsymbol{p}_{1}\right)}(\lambda, u)=\left\{G^{\left(\boldsymbol{p}_{1}\right)} U^{\left(\boldsymbol{p}_{1}\right)}(\lambda, u) \prod^{p_{1}}\left(\lambda-u_{l}\right)^{T^{(l)}} \quad \prod^{p_{1}}\left(\lambda-u_{j}\right)^{R^{(j)}}\right\} \\
& \begin{array}{cc}
l=1 & j=q_{1}+1 \\
l \neq i & j \neq i
\end{array} \\
& \cdot\left(\lambda-u_{i}\right)^{T^{(i)}}\left(\lambda-u_{i}\right)^{R^{(i)}} \text {, }
\end{aligned}
$$

where it is understood that $R^{(i)}=0$ if $i=1, \ldots, q_{1}$. We have

$$
U^{\left(p_{1}\right)}(\lambda, u)=I+F_{i}(u)+O\left(\lambda-u_{i}\right), \quad \lambda \rightarrow u_{i}, \quad F_{i}(u):=U^{\left(p_{1}\right)}\left(u_{i}, u\right),
$$

and $O\left(\lambda-u_{i}\right)$ represent vanishing terms at $\lambda=u_{i}$, holomorphic in $\mathbb{D}_{1} \times \mathbb{D}\left(u^{c}\right)$. The expansion at $\lambda=u_{i}$ of the factors $\left(\lambda-u_{l}\right)^{T^{(i)}}$ and $\left(\lambda-u_{j}\right)^{R^{(j)}}$, for $l, j \neq i$, yields the Levelt form

$$
\Psi^{\left(\boldsymbol{p}_{1}\right)}(\lambda, u) \underset{\lambda \rightarrow u_{i}}{=} G^{\left(i ; \boldsymbol{p}_{1}\right)}(u)\left(I+O\left(\lambda-u_{i}\right)\right)\left(\lambda-u_{i}\right)^{T^{(i)}}\left(\lambda-u_{i}\right)^{R^{(i)}}, \quad i=1, \ldots, p_{1},
$$

where $O\left(\lambda-u_{i}\right)$ are higher order terms, provided that $u \in \mathbb{D}\left(u^{0}\right)$ (they contain negative powers $\left.\left(u_{i}-u_{k}\right)^{-m}\right)$, and

$$
G^{\left(i ; \boldsymbol{p}_{1}\right)}(u):=G^{\left(\boldsymbol{p}_{1}\right)}\left(I+F_{i}(u)\right) \prod_{\substack{l=1 \\ l \neq i}}^{p_{1}}\left(u_{i}-u_{l}\right)^{T^{(l)}} \prod_{\substack{j=q_{1}+1 \\ j \neq i}}^{p_{1}}\left(u_{i}-u_{j}\right)^{R^{(j)}}, i=1, \ldots, p_{1} .
$$

The matrix $G^{\left(i ; p_{1}\right)}(u)$ is holomorphically invertible if restricted to a polydisc $\mathbb{D}\left(u^{0}\right)$ contained in a $\tau$-cell, but it is branched at the coalescence locus $\Delta$ on the whole $\mathbb{D}\left(u^{c}\right)$.

We reach our goal if we show that the $i$ th column $G^{\left(i ; \boldsymbol{p}_{1}\right)}(u) \cdot \vec{e}_{i}$ is constant in $\mathbb{D}\left(u^{c}\right)$. First, it follows from (6.38) and the standard isomonodromic theory of [33] that $G^{\left(i ; p_{1}\right)}(u)$ holomorphically in $\mathbb{D}\left(u^{0}\right)$ reduces $B_{i}(u)$ to the diagonal form $T^{(i)}$, when $\lambda_{i}^{\prime} \neq-1$,

$$
\left(G^{\left(i ; \boldsymbol{p}_{1}\right)}(u)\right)^{-1} B_{i}(u) G^{\left(i ; \boldsymbol{p}_{1}\right)}(u)=T^{(i)},
$$

or to non-diagonal Jordan form (6.21) when $\lambda_{i}^{\prime}=-1$

$$
\left(G^{\left(i ; p_{1}\right)}(u)\right)^{-1} B_{i}(u) G^{\left(i ; p_{1}\right)}(u)=R^{(i)} \equiv J^{(i)}, \quad \lambda_{i}^{\prime}=-1 .
$$

For this reason, the $i$ th row is proportional to the eigenvector $\vec{e}_{i}$ of $B_{i}(u)$ relative to the eigenvalue $-\lambda_{i}^{\prime}-1$. Namely, for some scalar function $f_{i}(u)$,

$$
G^{\left(i ; p_{1}\right)}(u) \cdot \vec{e}_{i}=f_{i}(u) \vec{e}_{i} .
$$


This is obvious for $\lambda_{i}^{\prime} \neq-1$, namely for diagonalizable $B_{i}$. If $\lambda_{i}^{\prime}=-1$, the eigenvalue 0 of $B_{i}$ appearing in $J^{(i)}$ at entry $(i, i)$ is associated with the eigenvector $f_{i}(u) \vec{e}_{i}$. Moreover, for every invertible matrix $G=\left[*|\cdots| *\left|\vec{e}_{i}\right| *|\cdots| *\right]$, where $\vec{e}_{i}$ occupies the $k$ th column, then $G^{-1} B_{i}(u) G$ is zero everywhere, except for the $k$ th row. Now, since $R^{(i)}=J^{(i)}$ has only one non-zero entry on the $i$ th row, it follows that the eigenvector $f_{i}(u) \vec{e}_{i}$ must occupy the $i$ th column of $G^{\left(i ; p_{1}\right)}(u)$.

- $f_{i}(u)$ is holomorphic on $\mathbb{D}\left(u^{c}\right)$. Indeed, by (6.28),

$$
\prod_{\substack{l=1 \\ l \neq i}}^{p_{1}}\left(u_{i}-u_{l}\right)^{T^{(l)}} \prod_{\substack{j=q_{1}+1 \\ j \neq i}}^{p_{1}}\left(u_{i}-u_{j}\right)^{R^{(j)}} \cdot \vec{e}_{i}=\vec{e}_{i} .
$$

Therefore $f_{i}(u) \vec{e}_{i} \equiv G^{\left(i ; \boldsymbol{p}_{1}\right)}(u) \cdot \vec{e}_{i}=G^{\left(\boldsymbol{p}_{1}\right)}\left(I+F_{i}(u)\right) \vec{e}_{i}$. We conclude, because $F_{i}(u)$ is holomorphic on $\mathbb{D}\left(u^{c}\right)$.

- $f_{i}$ is constant on $\mathbb{D}\left(u^{c}\right)$. Indeed, since $\Psi^{\left(p_{1}\right)}(\lambda, u)$ is an isomonodromic solution in $\mathbb{D}\left(u^{0}\right)$, the matrix $G^{\left(i ; p_{1}\right)}(u)$ must satisfy the Pfaffian system (see Appendix A, identify $G^{\left(i ; p_{1}\right)}$ with $G^{(i)}$ in Corollary 9.1)

$$
\begin{aligned}
\frac{\partial G^{\left(i ; \boldsymbol{p}_{1}\right)}}{\partial u_{j}} & =\left(\frac{B_{j}}{u_{j}-u_{i}}+\omega_{j}\right) G^{\left(i ; \boldsymbol{p}_{1}\right)}, \quad j \neq i ; \quad \frac{\partial G^{\left(i ; \boldsymbol{p}_{1}\right)}}{\partial u_{i}} \\
& =\sum_{j \neq i}\left(\frac{B_{j}}{u_{i}-u_{j}}+\omega_{j}\right) G^{\left(i ; \boldsymbol{p}_{1}\right)}
\end{aligned}
$$

From (2.18) and (4.3), the $i$ th column of $\frac{B_{j}}{u_{j}-u_{i}}+\omega_{j}$ is null. Hence,

$$
\frac{\partial}{\partial u_{j}}\left(G^{\left(i ; \boldsymbol{p}_{1}\right)} \cdot \vec{e}_{i}\right)=0, \quad \forall j \neq i
$$

Moreover, summing the Eq. (6.39), we get $\sum_{j=1}^{n} \partial_{j} G^{\left(i ; \boldsymbol{p}_{1}\right)}=0$. Thus, $G^{\left(i ; \boldsymbol{p}_{1}\right)} \cdot \vec{e}_{i}$ is constant on $\mathbb{D}\left(u^{0}\right)$, and being holomorphic on $\mathbb{D}\left(u^{c}\right)$, it is constant on $\mathbb{D}\left(u^{c}\right)$. The choice (6.37) will be made.

The above obtained expansions for the $\vec{\Psi}_{i}$ and $\vec{\Psi}_{i}^{(\operatorname{sing})}$ and $\vec{\varphi}_{i}$ prove Theorem 5.1 for $i=1, \ldots, p_{1}$, with some obvious identifications between objects in the proof and objects in the statement, namely $\vec{\varphi}_{i} \longmapsto \vec{\psi}_{i}, r_{i}^{(m)} / r_{k}^{(i)} \longmapsto r_{m}$ and $\vec{\varphi}_{k} / r_{k}^{(i)} \longmapsto \phi_{i}$.

\subsection{Analogous proof for all coalescences}

With the labelling (6.1)-(6.2), the same strategy above holds for every coalescence

$$
\left(u_{p_{1}+\cdots+p_{\alpha-1}+1}, \ldots, u_{p_{1}+\cdots+p_{\alpha}}\right) \longrightarrow\left(\lambda_{\alpha}, \ldots, \lambda_{\alpha}\right), \quad \alpha=1, \ldots, s .
$$


We find corresponding isomonodromic fundamental matrices for the Pfaffian system (with self-explaining notations)

$$
\begin{gathered}
\Psi^{\left(\boldsymbol{p}_{\alpha}\right)}(\lambda, u)=G^{\left(\boldsymbol{p}_{\alpha}\right)} \cdot U^{\left(\boldsymbol{p}_{\alpha}\right)}(\lambda, u) \cdot \prod_{l=p_{1}+\cdots+p_{\alpha-1}+1}^{p_{1}+\cdots+p_{\alpha}}\left(\lambda-u_{l}\right)^{T^{(l)}} \\
\prod_{j=\left(p_{1}+\cdots+p_{\alpha-1}+1\right)+q_{\alpha}}^{p_{1}+\cdots+p_{\alpha}}\left(\lambda-u_{j}\right)^{R^{(j)}} \cdot
\end{gathered}
$$

where $\boldsymbol{p}_{\alpha}=\left(p_{1}+\cdots+p_{\alpha-1}+1, \ldots, p_{1}+\cdots+p_{\alpha}\right)$. Then, we proceed in the same way, constructing the solutions $\vec{\Psi}_{i}$ and $\vec{\Psi}_{i}^{(\text {sing })}$, with $p_{1}+\cdots+p_{\alpha-1}+1 \leq i \leq$ $p_{1}+\cdots+p_{\alpha}$.

\subsection{Proof of Proposition 5.1}

Proof For simplicity, we omit $v$ in the connection coefficients $c_{j k}^{(v)}$ in (5.12)-(5.13). It follows from the very definitions of the $\vec{\Psi}_{k}$ and $\vec{\Psi}_{j}^{(\text {sing })}$ that

$$
c_{j k}=0 \quad \text { if } u_{j}^{c}=u_{k}^{c} .
$$

In order to prove independence of $u$, we express the monodromy of

$$
\Psi(\lambda, u):=\left[\vec{\Psi}_{1}(\lambda, u)|\cdots| \vec{\Psi}_{n}(\lambda, u)\right]
$$

in terms of the connection coefficients. From the definition, we have (using the notations in the statement of Theorem 5.1)

$$
\vec{\Psi}_{k}(\lambda, u)= \begin{cases}\vec{\Psi}_{j}(\lambda, u) c_{j k}+\operatorname{reg}\left(\lambda-u_{j}\right), & \lambda_{j}^{\prime} \notin \mathbb{Z} \\ \vec{\Psi}_{j}(\lambda, u) \ln \left(\lambda-u_{j}\right) c_{j k}+\operatorname{reg}\left(\lambda-u_{j}\right), & \lambda_{j}^{\prime} \in \mathbb{Z}_{-} \\ \left(\vec{\Psi}_{j}(\lambda, u) \ln \left(\lambda-u_{j}\right)+\frac{\psi_{j}(\lambda, u)}{\left(\lambda-u_{j}\right)^{\lambda_{j}^{\prime}+1}}\right) c_{j k}+\operatorname{reg}\left(\lambda-u_{j}\right), \lambda_{j}^{\prime} \in \mathbb{N}\end{cases}
$$

For $u \notin \Delta$ and a small loop $\left(\lambda-u_{k}\right) \mapsto\left(\lambda-u_{k}\right) e^{2 \pi i}$ we obtain from Theorem 5.1

$$
\vec{\Psi}_{k}(\lambda, u) \longmapsto \vec{\Psi}_{k}(\lambda, u) e^{-2 \pi i \lambda_{k}^{\prime}}, \quad \text { which includes also the case } \lambda_{k}^{\prime} \in \mathbb{Z} \text {, with } e^{-2 \pi i \lambda_{k}^{\prime}}=1 \text {. }
$$

For a small loop $\left(\lambda-u_{j}\right) \mapsto\left(\lambda-u_{j}\right) e^{2 \pi i}, j \neq k$, from Theorem 5.1 and (6.40) we obtain

$$
\vec{\Psi}_{k} \longmapsto \vec{\Psi}_{j} e^{-2 \pi i \lambda_{j}^{\prime}} c_{j k}+\underbrace{\operatorname{reg}\left(\lambda-u_{j}\right)}_{\vec{\Psi}_{k}-\vec{\Psi}_{j} c_{j k}}=\vec{\Psi}_{k}+\left(e^{-2 \pi i \lambda_{j}^{\prime}}-1\right) c_{j k} \vec{\Psi}_{j} \quad \quad \text { for } \lambda_{j}^{\prime} \notin \mathbb{Z}
$$




$$
\begin{array}{ll}
\vec{\Psi}_{k} \longmapsto \vec{\Psi}_{j}\left(\ln \left(\lambda-u_{j}\right)+2 \pi i\right) c_{j k}+\operatorname{reg}\left(\lambda-u_{j}\right)=\vec{\Psi}_{k}+2 \pi i c_{j k} \vec{\Psi}_{j}, & \text { for } \lambda_{j}^{\prime} \in \mathbb{Z}_{-} \\
\vec{\Psi}_{k} \longmapsto\left(\vec{\Psi}_{j}\left(\ln \left(\lambda-u_{j}\right)+2 \pi i\right)+\frac{\psi_{j}(\lambda, u)}{\left(\lambda-u_{j}\right)^{\lambda_{j}^{\prime}+1}}\right) c_{j k}+\operatorname{reg}\left(\lambda-u_{j}\right)=\vec{\Psi}_{k}+2 \pi i c_{j k} \vec{\Psi}_{j}, & \text { for } \lambda_{j}^{\prime} \in \mathbb{N} .
\end{array}
$$

Therefore, for $u \notin \Delta$ and a small loop $\gamma_{k}:\left(\lambda-u_{k}\right) \mapsto\left(\lambda-u_{k}\right) e^{2 \pi i}$ not encircling other points $u_{j}$ (we denote the loop by $\lambda \mapsto \gamma_{k} \lambda$ ), we receive

$$
\Psi(\lambda, u) \longmapsto \Psi\left(\gamma_{k} \lambda, u\right)=\Psi(\lambda, u) M_{k}(u),
$$

where

$$
\begin{aligned}
& \left(M_{k}\right)_{j j}=1 \quad j \neq k, \quad\left(M_{k}\right)_{k k}=e^{-2 \pi i \lambda_{k}^{\prime}} ; \quad\left(M_{k}\right)_{k j}=\alpha_{k} c_{k j}, \quad j \neq k \\
& \left(M_{k}\right)_{i j}=0 \text { otherwise. }
\end{aligned}
$$

and

$$
\alpha_{k}:=\left(e^{-2 \pi i \lambda_{k}^{\prime}}-1\right), \quad \text { if } \lambda_{k}^{\prime} \notin \mathbb{Z} ; \quad \alpha_{k}:=2 \pi i, \quad \text { if } \lambda_{k}^{\prime} \in \mathbb{Z} .
$$

We proceed by first analyzing the generic case, and then the general case.

Generic case. Suppose that $A(u)$ has no integer eigenvalues (recall that eigenvalues do not depend on $u$ ). Let us fix $u$ in a $\tau$-cell. By Proposition 2.4, $\Psi(\lambda, u)$ is a fundamental matrix solution of (1.4) for the fixed $u$, and $C=\left(c_{j k}\right)$ is invertible. Thus

$$
M_{k}(u)=\Psi\left(\gamma_{k} \lambda, u\right) \Psi(\lambda, u)^{-1}
$$

The above makes sense for every $u$ in the considered $\tau$-cell, being $\Psi(\lambda, u)$ invertible at such an $u$. But $\Psi(\lambda, u)$ and $\Psi\left(\gamma_{k} \lambda, u\right)$ are holomorphic on $\mathcal{P}_{\eta}(u) \hat{\times} \mathbb{D}\left(u^{c}\right)$, so that the matrix $M_{k}(u)$ is holomorphic on the $\tau$-cell. Repeating the above argument for another $\tau$-cell, we conclude that $M_{k}(u)$ is holomorphic on each $\tau$-cell. Now, on a $\tau$-cell, we have

$$
\mathrm{d} \Psi\left(\gamma_{k} \lambda, u\right)=P(\lambda, u) \Psi\left(\gamma_{k} \lambda, u\right)=P(\lambda, u) \Psi(\lambda, u) M_{k},
$$

and at the same time

$$
\begin{aligned}
\mathrm{d} \Psi\left(\gamma_{k} \lambda, u\right) & =\mathrm{d}\left(\Psi(\lambda, u) M_{k}\right)=\mathrm{d} \Psi(\lambda, u) M_{k}+\Psi(\lambda, u) d M_{k} \\
& =P(\lambda, u) \Psi(\lambda, u) M_{k}+\Psi(\lambda, u) d M_{k}
\end{aligned}
$$

The two expressions are equal if and only if $d M_{k}=0$, because $\Psi(\lambda, u)$ is invertible on a $\tau$-cell. Recall that $\tau$-cells are disconnected from each other, so that separately on each cell, $M_{k}$ is constant, and so the connection coefficients are constant separately on each cell. 
We further suppose that none of the $\lambda_{j}^{\prime}$ is integer. In this case, $\vec{\Psi}_{j}^{(\text {sing })}=\vec{\Psi}_{j}$ for all $j=1, \ldots, n$, so that from (6.40) for $u_{k}^{c} \neq u_{j}^{c}$ (otherwise $c_{j k}=0$ and there is nothing to prove)

$$
\vec{\Psi}_{k}(\lambda, u) \underset{\lambda \rightarrow u_{j}}{=} \vec{\Psi}_{j}(\lambda, u) c_{j k}+\operatorname{reg}\left(\lambda-u_{j}\right) .
$$

Using the labelling (6.1)-(6.2), from the Proof of Theorem 6.1 we have the fundamental matrix solution

$$
\Psi^{\left(p_{1}\right)}(\lambda, u)=\left[\vec{\Psi}_{1}(\lambda, u)|\ldots| \vec{\Psi}_{p_{1}}(\lambda, u)\left|\vec{\varphi}_{p_{1}+1}^{(1)}(\lambda, u)\right| \cdots \mid \vec{\varphi}_{n}^{(1)}(\lambda, u)\right]
$$

and in general at each $\lambda_{\alpha}, \alpha=1, \ldots, s$ (with $\sum_{j=1}^{\alpha-1} p_{j}=0$ for $\alpha=1$ ) we have

$$
\begin{aligned}
& \Psi^{\left(\boldsymbol{p}_{\alpha}\right)}(\lambda, u) \\
& =\left[\vec{\varphi}_{1}^{(\alpha)}(\lambda, u)|\cdots| \vec{\varphi}_{\sum_{j=1}^{\alpha-1} p_{j}}^{(\alpha)}(\lambda, u)\left|\vec{\Psi}_{\sum_{j=1}^{\alpha-1} p_{j}+1}(\lambda, u)\right| \vec{\Psi}_{\sum_{j=1}^{\alpha-1} p_{j}+2}(\lambda, u) \mid\right. \\
& \quad \ldots\left|\vec{\Psi}_{\sum_{j=1}^{\alpha} p_{j}}(\lambda, u)\right| \\
& \quad \mid \vec{\varphi}_{\sum_{j=1}^{\alpha}(\alpha)}^{\alpha} p_{j}+1 \\
& \left.\quad(\lambda, u)|\cdots| \vec{\varphi}_{n}^{(\alpha)}(\lambda, u)\right]
\end{aligned}
$$

where

$$
\vec{\Psi}_{m}(\lambda, u)=\vec{\psi}_{m}(\lambda, u)\left(\lambda-u_{m}\right)^{-\lambda_{m}^{\prime}-1}, \quad m=\sum_{j=1}^{\alpha-1} p_{j}+1, \ldots, \sum_{j=1}^{\alpha} p_{j},
$$

and the $\vec{\psi}_{m}(\lambda, u)$ and $\vec{\varphi}_{r}{ }^{(\alpha)}(\lambda, u)$ are holomorphic functions in the corresponding $\mathbb{D}_{\alpha} \times \mathbb{D}\left(u^{c}\right)$. The above allows us to explicitly rewrite (6.41), for $j$ such that $u_{j}^{c}=\lambda_{\alpha}$, as

$$
\vec{\Psi}_{k}(\lambda, u)=\sum_{m=p_{1}+\cdots+p_{\alpha-1}+1}^{p_{1}+\cdots+p_{\alpha}} c_{m k} \vec{\Psi}_{m}(\lambda, u)+\sum_{r \notin\left\{p_{1}+\cdots+p_{\alpha-1}+1, \ldots, p_{1}+\cdots+p_{\alpha}\right\}} h_{r} \vec{\varphi}_{r}^{(\alpha)}(\lambda, u),
$$

for suitable constant coefficients $h_{r}$. Here one of the $c_{m k}$ is $c_{j k}$ of (6.41).

Each $u_{m}$, with $m=p_{1}+\cdots+p_{\alpha-1}+1, \ldots, p_{1}+\cdots+p_{\alpha}$, varies in $\mathbb{D}_{\alpha}$. Firstly, we can fix $\lambda=\lambda_{\alpha}$ in (6.42), consider the branch cut $\mathcal{L}_{\alpha}$ from $\lambda_{\alpha}$ to infinity in direction $\eta$ (see Fig. 3), and let $u$ vary in such a way that each $u_{p_{1}+\cdots+p_{\alpha-1}+1}$, $\ldots, u_{p_{1}+\cdots+p_{\alpha}}$ varies in $\mathbb{D}_{\alpha} \backslash \mathcal{L}_{\alpha}$, so that in the r.h.s. of (6.42) all the $\vec{\Psi}_{m}\left(\lambda_{\alpha}, u\right)$ and $\vec{\varphi}_{r}{ }^{(\alpha)}\left(\lambda_{\alpha}, u\right)$ are holomorphic with respect to $u$, provided that $u_{m} \neq \lambda_{\alpha}$. If $u$ varies, with the constraint that the $u_{m}$ 's must remain in $\mathbb{D}_{\alpha} \backslash \mathcal{L}_{\alpha}$, every $\tau$-cell of $\mathbb{D}\left(u^{c}\right)$ can be reached starting from an initial point in one specific cell. This proves, by $u$-analytic continuation of (6.42) with fixed $\lambda=\lambda_{\alpha}$, that the coefficients $c_{m k}$ are constant ${ }^{16}$ in $\left(\mathbb{D}_{\alpha} \backslash \mathcal{L}_{\alpha}\right)^{\times p_{\alpha}} \times\left(\times_{\beta \neq \alpha} \mathbb{D}_{\beta}^{\times p_{\beta}}\right) \subset \mathbb{D}\left(u^{c}\right)$.

$\overline{16 \text { Recall that } \mathbb{D}\left(u^{c}\right)=\times_{\beta=1}^{s} \mathbb{D}_{\beta}} \times p_{\beta}$. 
Now, we can slightly vary $\eta$ in $\eta_{\nu+1}<\eta<\eta_{\nu}$, so that the cut $\mathcal{L}_{\alpha}$ is irrelevant ${ }^{17}$. Thus, the $c_{m k}$ are constant on $\left\{u \in \mathbb{D}\left(u^{c}\right) \mid u_{p_{1}+\cdots+p_{\alpha-1}+1} \neq \lambda_{\alpha}, \ldots, u_{p_{1}+\cdots+p_{\alpha}} \neq \lambda_{\alpha}\right\}$.

Finally, we fix another value $\lambda=\lambda^{*} \in \mathbb{D}_{\alpha}$ in (6.42), and repeat the above discussion with cuts $\mathcal{L}_{\alpha}$ issuing from $\lambda^{*}$, so that all the $c_{m k}$ are constant on $\left\{u \in \mathbb{D}\left(u^{c}\right) \mid u_{p_{1}+\cdots+p_{\alpha-1}+1} \neq \lambda^{*}, \ldots, u_{p_{1}+\cdots+p_{\alpha}} \neq \lambda^{*}\right\}$. This proves constancy of the $c_{m k}, m$ associated with $\lambda_{\alpha}$, on the whole $\mathbb{D}\left(u^{c}\right)$. Then, we repeat this for all $\alpha=1, . ., s$, proving constancy of the $c_{j k}$ for all $j=1, \ldots, n$. Hence, Proposition 5.1 is proved in the generic case.

General case of any $A(u)$. If some of the diagonal entries $\lambda_{1}^{\prime}, \ldots, \lambda_{n}^{\prime}$ of $A$ are integers, or some eigenvalues are integers, there exists a sufficiently small $\gamma_{0}>0$ such that, for any $0<\gamma<\gamma_{0}, A-\gamma I$ has diagonal non-integer entries $\lambda_{1}^{\prime}-\gamma, \ldots, \lambda_{n}^{\prime}-\gamma$ and no integer eigenvalues. Take such a $\gamma_{0}$, and for any $0<\gamma<\gamma_{0}$ consider

$$
(\Lambda-\lambda) \frac{\mathrm{d}}{\mathrm{d} \lambda}(\gamma \Psi)=((A(u)-\gamma I)+I) \gamma \Psi
$$

namely

$$
\frac{\mathrm{d}}{\mathrm{d} \lambda}(\gamma \Psi)=\sum_{k=1}^{n} \frac{B_{k}[\gamma](u)}{\lambda-u_{k}} \gamma \Psi, \quad B_{k}[\gamma](u):=-E_{k}(A(u)+(1-\gamma) I) .
$$

Lemma 6.5 The above system $(6.44)$ is strongly isomonodromic in $\mathbb{D}\left(u^{0}\right)$ contained in a $\tau$-cell, and $\lambda$-component of the integrable Pfaffian system

$d_{\gamma} \Psi=P_{[\gamma]}(\lambda, u)_{\gamma} \Psi, \quad P_{[\gamma]}(\lambda, u)=\sum_{k=1}^{n} \frac{B_{k}[\gamma](u)}{\lambda-u_{k}} d\left(\lambda-u_{k}\right)+\sum_{j=1}^{n}\left[F_{1}(u), E_{j}\right] \mathrm{d} u_{j}$.

where $F_{1}(u)$ is defined as in (2.8), $\left(F_{1}\right)_{i j}=\frac{A_{i j}}{u_{j}-u_{i}}, i \neq j$, and $\left[F_{1}(u), E_{j}\right]$ is (2.18).

Proof We do a gauge transformation

$$
\gamma Y(z):=z^{-\gamma} Y(z), \quad \gamma \in \mathbb{C}
$$

which transforms (1.1) into

$$
\frac{\mathrm{d}(\gamma Y)}{\mathrm{d} z}=\left(\Lambda+\frac{A-\gamma I}{z}\right) \gamma_{\gamma}
$$

For $u \in \mathbb{D}\left(u^{0}\right)$ contained in a $\tau$-cell, we write the unique formal solution

$$
{ }_{\gamma} Y_{F}(z, u)=z^{-\gamma} Y_{F}(z, u)
$$

where $Y_{F}(z, u)$ is $(2.4)$, so that

$$
{ }_{\gamma} Y_{F}(z, u)=F(z, u) z^{B-\gamma I} e^{\Lambda z}, \quad B-\gamma I=\operatorname{diag}(A-\gamma)=\operatorname{diag}\left(\lambda_{1}^{\prime}-\gamma, \ldots, \lambda_{n}^{\prime}-\gamma\right) .
$$

\footnotetext{
17 The crossing locus $X(\tau), \tau=3 \pi / 2-\eta$, is as arbitrary as is the choice of $\tau$ in the range $\tau_{\nu}<\tau<\tau_{\nu+1}$.
} 
The crucial point is that $F(z, u)$ is the same as $(2.5)$, so all the $F_{k}(u)$ are independent of $\gamma$. The fundamental matrix solutions

$$
{ }_{\gamma} Y_{\nu}(z, u):=z^{-\gamma} Y_{\nu}(z, u)
$$

are uniquely defined by their asymptotics ${ }_{\gamma} Y_{F}(z, u)$ in $\mathcal{S}_{v}\left(\mathbb{D}\left(u^{0}\right)\right)$. Their Stokes matrices do not depend on $\gamma$ because

$$
{ }_{\gamma} Y_{\nu+(k+1) \mu}(z, u)={ }_{\gamma} Y_{\nu+k \mu}(z, u) \mathbb{S}_{\nu+k \mu} \Longleftrightarrow Y_{\nu+(k+1) \mu}(z, u)=Y_{\nu+k \mu}(z, u) \mathbb{S}_{\nu+k \mu}
$$

The system (6.47) is thus strongly isomonodromic. By Proposition 3.1 we conclude.

Corollary 6.2 Let the assumptions of Theorem 5.1 hold. Then Theorem 5.1 holds also for (6.45).

By Theorem 5.1 applied to (6.45), we receive independent vector solutions $\gamma \vec{\Psi}_{k}(\lambda, u) \equiv \gamma \vec{\Psi}_{k}^{(s i n g)}(\lambda, u), k=1, \ldots, n$, which form a fundamental matrix

$$
{ }_{\gamma} \Psi(\lambda, u):=\left[\gamma \vec{\Psi}_{1}(\lambda, u)|\cdots|_{\gamma} \vec{\Psi}_{n}(\lambda, u)\right] .
$$

For system (6.45) the results already proved in the generic case hold. Therefore, the connection coefficients $c_{j k}^{(\nu)}[\gamma]$ defined by

$$
\gamma \vec{\Psi}_{k}(\lambda, u \mid v)={ }_{\gamma} \vec{\Psi}_{j}(\lambda, u \mid v) c_{j k}^{(v)}[\gamma]+\operatorname{reg}\left(\lambda-u_{j}\right), \quad \lambda \in \mathcal{P}_{\eta},
$$

are constant on $\mathbb{D}\left(u^{c}\right)$. They depend on $\gamma$, but not on $u \in \mathbb{D}\left(u^{c}\right)$.

Remark 6.4 It is explained in section 8 of [23] what is the relation between $\vec{\Psi}_{k}^{(s i n g)}$ and $\gamma \vec{\Psi}_{k}$, by means of their primitives, and that in general both $\lim _{\gamma \rightarrow 0} \vec{\Psi}_{k}^{k}$ and $\lim _{\gamma \rightarrow 0} c_{j k}^{(\nu)}[\gamma]$ are divergent.

Now, we invoke Proposition 10 of [23], which holds with no assumptions on eigenvalues and diagonal entries of $A(u) .{ }^{18}$ This result, adapted to our case, reads as follows.

Proposition 6.3 Let $u$ be fixed in a $\tau$-cell. Let $\gamma_{0}>0$ be small enough such that for any $0<\gamma<\gamma_{0}$ the matrix $A-\gamma I$ has no integer eigenvalues, and its diagonal part has no integer entries. ${ }^{19}$ Let $c_{j k}^{(v)}$ be the connection coefficients of the Fuchsian system (1.4) at the fixed $u$, as in Definition 5.1. Let $c_{j k}^{(v)}[\gamma]$ be the connection coefficients in (6.49). Let

$$
\alpha_{k}:=\left\{\begin{array}{cc}
e^{-2 \pi i \lambda_{k}^{\prime}}-1, & \lambda_{k}^{\prime} \notin \mathbb{Z} \\
2 \pi i, & \lambda_{k}^{\prime} \in \mathbb{Z}
\end{array} ; \quad \alpha_{k}[\gamma]:=e^{-2 \pi i\left(\lambda_{k}^{\prime}-\gamma\right)}-1\right.
$$

\footnotetext{
18 The proof in [23] is laborious, because it is necessary to take into account all possible values of the diagonal entries $\lambda_{k}^{\prime}$ of $A$, including integer values. In [4] the proof is given only for non-integer values.

19 Recall that eigenvalues and diagonal entries do not depend on $u$, in the isomonodromic case.
} 
Then, the following equalities hold

$$
\alpha_{k} c_{j k}^{(v)}=e^{-2 \pi i \gamma} \alpha_{k}[\gamma] c_{j k}^{(v)}[\gamma], \quad \text { if } k \succ j ; \quad \alpha_{k} c_{j k}^{(v)}=\alpha_{k}[\gamma] c_{j k}^{(v)}[\gamma], \quad \text { if } k \prec j ;
$$

where the ordering relation $j \prec k$ means, for the fixed $u$, that $\Re\left(z\left(u_{j}-u_{k}\right)\right)<0$ for $\arg z=\tau=3 \pi / 2-\eta$ satisfying (5.2).

We use Proposition 6.3 to conclude the proof of Proposition 5.1 in the general case. Indeed, the proposition is already proved in the generic case, so it holds for the $c_{j k}^{(v)}[\gamma]$. Therefore, they are constant on the whole $\mathbb{D}\left(u^{c}\right)$. Equalities (6.50) hold at any fixed $u$ in $\tau$-cell, so that each $c_{j k}^{(v)}$ is constant on a $\tau$-cell, and such constant is the same in each $\tau$-cell. With a slight variation of $\eta$ in $\left(\eta_{\nu+1}, \eta_{\nu}\right)$, equalities (6.50) hold also at the crossing locus $X(\tau)$. They analytically extend at $\Delta$.

\section{Laplace transform in $\mathbb{D}\left(u^{c}\right)$, Theorem 7.1}

By means of the Laplace transform with deformation parameters, we prove points (I1),(I2), (I3), (II1), (II2) and (II5) of Theorem 2.2. Stokes matrices will be expressed in terms of the isomonodromic connection coefficients satisfying Proposition 5.1. The result is in Theorem 7.1, which is the last step of our construction.

Let $\tau$ be the chosen direction in the $z$-plane admissible at $u^{c}$, and $\eta=3 \pi / 2-\tau$ in the $\lambda$-plane. The Stokes rays of $\Lambda\left(u^{c}\right)$ will be labelled as in (2.21), so that (5.2) holds for a certain $v \in \mathbb{Z}$. We define the sectors

$$
\mathcal{S}_{v}=\left\{z \in \mathcal{R}(\mathbb{C} \backslash\{0\}) \text { such that } \tau_{v}-\pi<\arg z<\tau_{v+1}\right\} .
$$

If $u$ only varies in $\mathbb{D}\left(u^{0}\right)$ contained in a $\tau$-cell, then none of the Stokes rays associated with $\Lambda(u)$ crosses $\arg z=\tau \bmod \pi$. If $u$ varies in $\mathbb{D}\left(u^{c}\right)$, some Stokes rays associated with $\Lambda(u)$ necessarily cross $\arg z=\tau \bmod \pi$ (see Sect. 2.1.2). Consider the subset of the set of Stokes rays satisfying $\Re\left(z\left(u_{j}-u_{k}\right)\right)=0, z \in \mathcal{R}$, associated with pairs $\left(u_{j}, u_{k}\right)$ such that $u_{j} \in \mathbb{D}_{\alpha}$ and $u_{k} \in \mathbb{D}_{\beta}, \alpha \neq \beta$, namely $u_{j}^{c} \neq u_{k}^{c}$. Following [13], we denote this subset by $\Re(u)$. If $u$ varies in $\mathbb{D}\left(u^{c}\right)$ and $\epsilon_{0}$ satisfies (5.1), the rays in $\mathfrak{R}(u)$ continuously rotate, but never cross the admissible rays $\arg z=\tau+h \pi$, where

$$
\tau_{\nu+h \mu}<\tau+h \pi<\tau_{\nu+h \mu+1}, \quad h \in \mathbb{Z},
$$

The above allows to define $\widehat{\mathcal{S}}_{\nu+h \mu}(u)$ to be the unique sector containing $S(\tau+(h-$ 1) $\pi, \tau+h \pi)$ and extending up to the nearest Stokes rays in $\mathfrak{R}(u)$. Then, let

$$
\widehat{\mathcal{S}}_{\nu+h \mu}:=\bigcap_{u \in \mathbb{D}\left(u^{c}\right)} \widehat{\mathcal{S}}_{\nu+h \mu}(u) .
$$

It has angular amplitude greater than $\pi$. The reason for the labeling is that $\widehat{\mathcal{S}}_{\nu+h \mu}\left(u^{c}\right)=$ $\mathcal{S}_{v+h \mu}$ in (7.1). 
Suppose that $u$ is fixed in a $\tau$-cell. Let

$$
Y_{v+h \mu}(z, u):=\left[\vec{Y}_{1}(z, u \mid v+h \mu)|\ldots| \vec{Y}_{n}(z, u \mid v+h \mu)\right],
$$

be defined by

$$
\begin{array}{ll}
\vec{Y}_{k}(z, u \mid v+h \mu):=\frac{1}{2 \pi i} \int_{\gamma_{k}(\eta-h \pi)} e^{z \lambda} \vec{\Psi}_{k}^{(\text {sing })}(\lambda, u \mid v+h \mu) \mathrm{d} \lambda, & \text { for } \lambda_{k}^{\prime} \notin \mathbb{Z}_{-}, \\
\vec{Y}_{k}(z, u \mid v+h \mu):=\int_{L_{k}(\eta-h \pi)} e^{z \lambda} \vec{\Psi}_{k}(\lambda, u \mid v+h \mu) \mathrm{d} \lambda, & \text { for } \lambda_{k}^{\prime} \in \mathbb{Z}_{-} .
\end{array}
$$

In the $\lambda$-plane, the admissible directions $\eta-h \pi$ correspond to $\tau+h \pi$, with

$$
\eta_{v+h \mu+1}<\eta-h \pi<\eta_{\nu+h \mu}
$$

Here, $\vec{\Psi}_{k}(\lambda, u \mid v+h \mu), \vec{\Psi}_{k}^{(\text {sing })}(\lambda, u \mid v+h \mu)$ are the vector solutions of Theorem 5.1 for $\lambda \in \mathcal{P}_{\eta-h \pi}(u)$, with $u$ fixed in a $\tau$-cell. $L_{k}(\eta-h \pi)$ is the cut in direction $\eta-h \pi$, issuing from $u_{k}$ and oriented from $u_{k}$ to $\infty$, and $\gamma_{k}(\eta-h \pi)$ is the path coming from $\infty$ along the left side of $L_{k}(\eta-h \pi)$, encircling $u_{k}$ with a small loop excluding all the other poles, and going back to $\infty$ along the right side of $L_{k}(\eta-h \pi)$. Here "right" and "left" refer to the orientation of $L_{k}(\eta-h \pi)$. The label $v+h \mu$ keeps track of (5.2) and (7.2)-(7.6).

Theorem 7.1 Let the assumptions of Theorem 5.1 hold.

(1) The matrices $Y_{v+h \mu}(z, u)$, obtained by Laplace transform (7.4)-(7.5) at a fixed $u \in \mathbb{D}\left(u^{0}\right)$ contained in a $\tau$-cell, define holomorphic matrix valued functions of $(\lambda, u) \in \mathcal{R}(\mathbb{C} \backslash\{0\}) \times \mathbb{D}\left(u^{c}\right)$, which are fundamental matrix solutions of (1.1).

(2) They have structure

$$
Y_{\nu+h \mu}(z, u)=\widehat{Y}_{\nu+h \mu}(z, u) z^{B} e^{z \Lambda}, \quad B=\operatorname{diag}\left(\lambda_{1}^{\prime}, \ldots, \lambda_{n}^{\prime}\right)
$$

with asymptotic behaviour, uniform in $u \in \mathbb{D}\left(u^{c}\right)$,

$$
\widehat{Y}_{\nu+h \mu}(z, u) \sim F(z, u)=I+\sum_{l=1}^{\infty} \frac{F_{l}(u)}{z^{l}}, \quad z \rightarrow \infty \text { in } \widehat{S}_{v+h \mu}
$$

given by the formal solution $Y_{F}(z, u)=F(z, u) z^{B} e^{z \Lambda}$. The coefficients $F_{l}(u)$ are holomorphic in $\mathbb{D}\left(u^{c}\right)$. The explicit expression of their columns is (7.12), (7.13), (7.15) [or (7.16)] and (7.17). 
(3) Stokes matrices defined by

$$
Y_{\nu+(h+1) \mu}(z, u)=Y_{\nu+h \mu}(z, u) \mathbb{S}_{\nu+h \mu}, \quad z \in \widehat{\mathcal{S}}_{\nu+h \mu} \cap \widehat{\mathcal{S}}_{\nu+(h+1) \mu},
$$

are constant in the whole $\mathbb{D}\left(u^{c}\right)$ and satisfy

$$
\left(\mathbb{S}_{v+h \mu}\right)_{a b}=\left(\mathbb{S}_{v+h \mu}\right)_{b a}=0 \quad \text { for } a \neq b \text { such that } u_{a}^{c}=u_{b}^{c}
$$

(4) The following representation in terms of the constant connection coefficients $c_{j k}^{(v)}$ of Proposition 5.1 holds on $\mathbb{D}\left(u^{c}\right)$ :

$$
\begin{array}{r}
\left(\mathbb{S}_{\nu}\right)_{j k}=\left\{\begin{array}{lc}
e^{2 \pi i \lambda_{k}^{\prime} \alpha_{k} c_{j k}^{(v)},} & j \prec k, u_{j}^{c} \neq u_{k}^{c}, \\
1 & j=k, \\
0 & j \succ k, u_{j}^{c} \neq u_{k}^{c}, \\
0 & j \neq k, u_{j}^{c}=u_{k}^{c},
\end{array}\right. \\
\left(\mathbb{S}_{\nu+\mu}^{-1}\right)_{j k}= \begin{cases}0 & j \neq k, u_{j}^{c}=u_{k}^{c}, \\
0 & j \prec k, u_{j}^{c} \neq u_{k}^{c}, \\
1 & j=k, \\
-e^{2 \pi i\left(\lambda_{k}^{\prime}-\lambda_{j}^{\prime}\right)} \alpha_{k} c_{j k}^{(v)} & j \succ k, u_{j}^{c} \neq u_{k}^{c},\end{cases}
\end{array}
$$

where the relation $j \prec k$ is defined for $j \neq k$ such that $u_{j}^{c} \neq u_{k}^{c}$ and means that $\Re\left(z\left(u_{j}^{c}-u_{k}^{c}\right)\right)<0$ when $\arg z=\tau$.

Remark 7.1 The above (7.9) generalizes Theorem 2.3 in the presence of isomonodromic deformation parameters, including coalescences. Notice that the ordering relation $\prec$ here is referred to $u^{c}$, while in Theorem 2.3 it refers to $u^{0}$.

Proof We use the labelling (6.1)-(6.2) for $u^{c}$.

a) Case $\lambda_{k}^{\prime} \notin \mathbb{Z}$.

- Construction of $\vec{Y}_{k}(z, u \mid v)$. We have $\vec{\Psi}_{k}^{(s i n g)}(\lambda, u \mid v)=\vec{\Psi}_{k}(\lambda, u \mid v)$ and (7.4) is

$$
\vec{Y}_{k}(z, u \mid v):=\frac{1}{2 \pi i} \int_{\gamma_{k}(\eta)} e^{z \lambda} \vec{\Psi}_{k}(\lambda, u \mid v) \mathrm{d} \lambda
$$

Since $\vec{\Psi}_{k}(\lambda, u \mid v)$ grows at infinity no faster than some power of $\lambda$, the integral converges in a sector of amplitude at most $\pi$. Now, $\vec{\Psi}_{k}(\lambda, u \mid v)$ satisfies Theorem 5.1, hence if $u$ varies in $\mathbb{D}\left(u^{c}\right)$ the following facts hold.

(1) $\vec{\Psi}_{k}(\lambda, u \mid v)$ is branched at $\lambda=u_{k}$ and possibly at other poles $u_{l}$ such that $u_{l}^{c} \neq u_{k}^{c}$.

(2) $\vec{\Psi}_{k}(\lambda, u \mid v)$ is holomorphic at all $\lambda=u_{j}$ such that $u_{j}^{c}=u_{k}^{c}, j \neq k$.

It follows from (1) to (2) that the path of integration can be modified: for $\alpha$ such that $u_{k}^{c}=\lambda_{\alpha}$, we have

$$
\vec{Y}_{k}(z, u \mid v)=\frac{1}{2 \pi i} \int_{\Gamma_{\alpha}(\eta)} e^{z \lambda} \vec{\Psi}_{k}(\lambda, u \mid v) \mathrm{d} \lambda,
$$




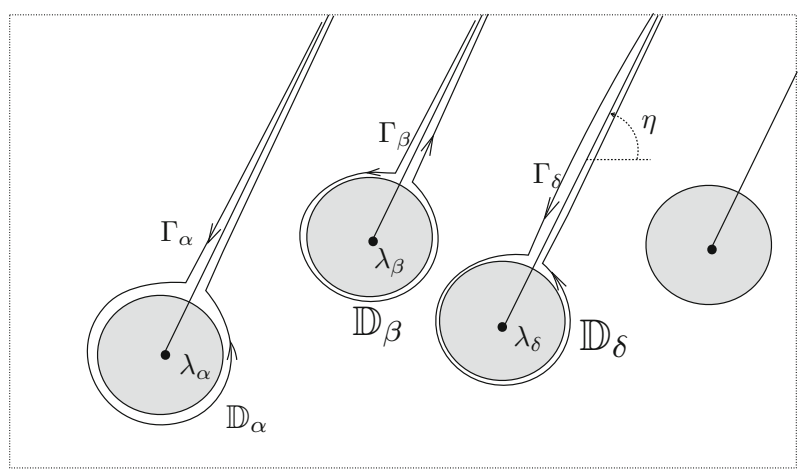

Fig. 4 The paths of integration $\Gamma_{\alpha}, \Gamma_{\beta}$, etc $\alpha, \beta, \ldots \in\{1, \ldots, s\}$

where $\Gamma_{\alpha}(\eta)$ is the path which comes from $\infty$ in direction $\eta-\pi$, encircles $\lambda_{\alpha}$ along $\partial \mathbb{D}_{\alpha}$ anti-clockwise and goes to $\infty$ in direction $\eta$. This path encloses all the $u_{j}$ such that $u_{j}^{c}=\lambda_{\alpha}$, end excludes the others. See Fig. 4. We conclude that $u$ can vary in $\mathbb{D}\left(u^{c}\right)$ and the integral (7.11) converges for $z$ in the sector

$$
\mathcal{S}(\eta):=\left\{z \in \mathcal{R}(\mathbb{C} \backslash\{0\}) \text { such that } \frac{\pi}{2}-\eta<\arg z<\frac{3 \pi}{2}-\eta\right\},
$$

defining $\vec{Y}_{k}(z, u \mid v)$ as a holomorphic function of $(z, u) \in \mathcal{S}(\eta) \times \mathbb{D}\left(u^{c}\right)$. Now, if $u$ varies in $\mathbb{D}\left(u^{c}\right)$ and $\epsilon_{0}$ satisfies (5.1) none of the vectors $u_{i}-u_{j}$ such that $u_{i}^{c}=\lambda_{\alpha}$ and $u_{j}^{c}=\lambda_{\beta}, 1 \leq \alpha \neq \beta \leq s$, cross a direction $\eta \bmod \pi$, for every $\eta_{\nu+1}<\eta<\eta_{\nu}$. Due to 1 . and 2. above, a vector function $\vec{\Psi}_{k}(\lambda, u \mid v)$ is well defined in $\mathcal{P}_{\eta}$ and $\mathcal{P}_{\tilde{\eta}}$ for any $\eta_{\nu+1}<\eta<\tilde{\eta}<\eta_{\nu}$, and so on $\mathcal{P}_{\eta} \cup \mathcal{P}_{\tilde{\eta}}$. Therefore, the integral in (7.11) satisfies

$\frac{1}{2 \pi i} \int_{\Gamma_{\alpha}(\eta)} e^{z \lambda} \vec{\Psi}_{k}(\lambda, u \mid v) \mathrm{d} \lambda=\frac{1}{2 \pi i} \int_{\Gamma_{\alpha}(\tilde{\eta})} e^{z \lambda} \vec{\Psi}_{k}(\lambda, u \mid v) \mathrm{d} \lambda, \quad z \in \mathcal{S}(\eta) \cap \mathcal{S}(\tilde{\eta})$,

namely one is the analytic continuation of the other, so defining the function $\vec{Y}_{k}(z, u \mid v)$ as analytic on $\widehat{\mathcal{S}}_{v} \times \mathbb{D}\left(u^{c}\right)$, where

$$
\widehat{\mathcal{S}}_{\nu}:=\bigcup_{\eta_{v+1}<\eta<\eta_{v}} \mathcal{S}(\eta)
$$

coincides with (7.3) (with $h=0)$.

Finally, notice that $\left.e^{\lambda z}(\lambda-\Lambda) \vec{\Psi}_{k}(\lambda, u \mid \nu)\right|_{\Gamma(\alpha)}=0$, due to the exponential factor. By (2.25), the vector solutions $\vec{Y}_{k}(z, u \mid v)$ satisfy system (1.1).

- Asymptotic behaviour. From (5.4)-(5.5), we write (7.11) as

$$
\vec{Y}_{k}(z, u \mid v)=\frac{1}{2 \pi i} \int_{\Gamma_{\alpha}(\eta)} e^{z \lambda}\left(\Gamma\left(\lambda_{j}^{\prime}+1\right) \vec{e}_{j}+\sum_{l \geq 1} \vec{b}_{l}^{(k)}(u)\left(\lambda-u_{k}\right)^{l}\right)\left(\lambda-u_{k}\right)^{-\lambda_{k}^{\prime}-1}, \mathrm{~d} \lambda .
$$


with holomorphic $\vec{b}_{l}^{(k)}(u)$ on $\mathbb{D}\left(u^{c}\right)$. We split the series as $\sum_{l \geq 1}=\sum_{l=1}^{\mathcal{N}}+\sum_{l \geq \mathcal{N}+1}$, and recall the standard formula (see [18])

$$
\int_{\Gamma_{\alpha}(\eta)}\left(\lambda-\lambda_{k}\right)^{a} e^{z \lambda} \mathrm{d} \lambda=\int_{\gamma_{k}(\eta)}\left(\lambda-\lambda_{k}\right)^{a} e^{z \lambda} \mathrm{d} \lambda=\frac{z^{-a-1} e^{\lambda_{k} z}}{\Gamma(-a)}
$$

so that

$$
\vec{Y}_{k}(z, u \mid v)=\left(\vec{e}_{k}+\sum_{l=1}^{\mathcal{N}} \frac{\vec{b}_{l}^{(k)}(u)}{\Gamma\left(\lambda_{k}^{\prime}+1-l\right)} z^{-l}+R_{\mathcal{N}}(z)\right) z^{\lambda_{k}^{\prime}} e^{\lambda_{k} z}
$$

with remainder

$$
R_{\mathcal{N}}(z)=\oint_{\Gamma_{0}(\eta)} \sum_{l \geq \mathcal{N}} \frac{\vec{b}_{l}^{(k)}(u)}{z^{l}} e^{x} x^{l-\lambda_{k}^{\prime}-1} \mathrm{~d} x=O\left(z^{-\mathcal{N}+1}\right)
$$

The integral is along a path $\Gamma_{0}(\eta)$, coming from $\infty$ along the left part of the half line oriented from 0 to $\infty$ in direction $\eta+\arg z$, going around 0 , and back to $\infty$ along the right part. The estimate $O\left(z^{-\mathcal{N}+1}\right)$ is standard. We conclude that

$$
\begin{aligned}
& \vec{Y}_{k}(z, u \mid v)\left(z^{\lambda_{k}^{\prime}} e^{u_{k} z}\right)^{-1} \sim \vec{e}_{k}+\sum_{l=1}^{\infty} \frac{\vec{b}_{l}^{(k)}(u)}{\Gamma\left(\lambda_{k}^{\prime}+1-l\right)} z^{-l} \equiv \vec{e}_{k}+\sum_{l=1}^{\infty} \vec{f}_{l}^{(k)}(u) z^{-l}, \\
& \quad z \rightarrow \infty \text { in } \widehat{\mathcal{S}}_{v}
\end{aligned}
$$

with

$$
\vec{f}_{l}^{(k)}(u):=\frac{\vec{b}_{l}^{(k)}(u)}{\Gamma\left(\lambda_{k}^{\prime}+1-l\right)} .
$$

b) Case $\lambda_{k}^{\prime} \in \mathbb{N}=\{0,1,2, \ldots\}$.

- Construction of $\vec{Y}_{k}(z, u \mid v)$. Definition (7.4) is

$$
\begin{aligned}
\vec{Y}_{k}(z, u \mid v): & =\frac{1}{2 \pi i} \int_{\gamma_{k}(\eta)} e^{z \lambda} \vec{\Psi}_{k}^{(\operatorname{sing})}(\lambda, u \mid v) \mathrm{d} \lambda \\
& =\frac{1}{(5.10)} \frac{\vec{\psi}_{k}(\lambda, u \mid v)}{2 \pi i} \int_{\gamma_{k}(\eta)} e^{z \lambda}\left(\frac{\left.\vec{\Psi}_{k}(\lambda, u \mid v) \ln \left(\lambda-u_{k}\right)\right) \mathrm{d} \lambda}{\left(\lambda-u_{k}\right)^{\lambda_{k}^{\prime}+1}}\right.
\end{aligned}
$$

The same facts 1 . and 2 . of the previous case apply to $\vec{\Psi}_{k}(\lambda, u \mid v)$ and $\vec{\psi}_{k}(\lambda, u \mid v)$ and allow to rewrite

$$
\vec{Y}_{k}(z, u \mid v)=\frac{1}{2 \pi i} \int_{\Gamma_{\alpha}(\eta)} e^{z \lambda}\left(\frac{\vec{\psi}_{k}(\lambda, u \mid v)}{\left(\lambda-u_{k}\right)^{\lambda_{k}^{\prime}+1}}+\vec{\Psi}_{k}(\lambda, u \mid v) \ln \left(\lambda-u_{k}\right)\right) \mathrm{d} \lambda
$$




$$
=\frac{1}{2 \pi i} \int_{\Gamma_{\alpha}(\eta)} e^{z \lambda} \vec{\Psi}_{k}^{(\text {sing })}(\lambda, u \mid v) \mathrm{d} \lambda .
$$

We conclude that $\vec{Y}_{k}(z, u \mid v)$ is analytic on $\widehat{\mathcal{S}}_{v} \times \mathbb{D}\left(u^{c}\right)$. Moreover, $e^{\lambda z}(\lambda-$ $\Lambda)\left.\vec{\Psi}_{k}^{(\text {sing })}(\lambda, u \mid v)\right|_{\Gamma(\alpha)}=0$, due to the exponential factor. By (2.25), the vector solution $\vec{Y}_{k}(z, u \mid v)$ satisfies the system (1.1).

- Asymptotic behaviour. By (5.7) and (5.11), and the fact that $\vec{\psi}_{k}$ has no singularities at $u_{j} \in \mathbb{D}_{\alpha}, j \neq k$, so that the terms $\sum_{l \geq 1+\lambda_{k}^{\prime}} \vec{b}_{l}^{(k)}(u)\left(\lambda-u_{k}\right)^{l}$ in $\vec{\psi}_{k}(\lambda, u \mid v)$ do not contribute to the integration, we can write

$$
\begin{aligned}
& \vec{Y}_{k}(z, u \mid v) \\
& \quad=\frac{1}{2 \pi i} \int_{\Gamma_{\alpha}(\eta)}\left(\frac{\lambda_{k}^{\prime} ! \vec{e}_{k}+\sum_{l=1}^{\lambda_{k}^{\prime}} \vec{b}_{l}^{(k)}(u)\left(\lambda-u_{k}\right)^{l}}{\left(\lambda-u_{k}\right)^{\lambda_{k}^{\prime}+1}}+\sum_{l=0}^{\infty} \vec{d}_{l}^{(k)}(u)\left(\lambda-u_{k}\right)^{l} \ln \left(\lambda-u_{k}\right)\right) e^{z \lambda} \mathrm{d} \lambda .
\end{aligned}
$$

By Cauchy formula

$$
\begin{aligned}
& \frac{1}{2 \pi i} \int_{\Gamma_{\alpha}(\eta)}\left(\frac{\lambda_{k}^{\prime} ! \vec{e}_{k}+\sum_{l=1}^{\lambda_{k}^{\prime}} \vec{b}_{l}^{(k)}(u)\left(\lambda-u_{k}\right)^{l}}{\left(\lambda-u_{k}\right)^{\lambda_{k}^{\prime}+1}}\right) e^{z \lambda} \mathrm{d} \lambda \\
& \quad=\left.\frac{1}{\lambda_{k}^{\prime} !} \frac{\mathrm{d}^{\lambda_{k}^{\prime}}}{\mathrm{d} \lambda^{\lambda_{k}^{\prime}}}\left[\left(\lambda_{k}^{\prime} ! \vec{e}_{k}+\sum_{l=1}^{\lambda_{k}^{\prime}} \vec{b}_{l}^{(k)}(u)\left(\lambda-u_{k}\right)^{l}\right) e^{z \lambda}\right]\right|_{\lambda=u_{k}} \\
& =z^{\lambda_{k}^{\prime}} e^{u_{k} z}\left(\vec{e}_{k}+\sum_{l=1}^{\lambda_{k}^{\prime}} \vec{f}_{l}^{(k)}(u) \frac{1}{z^{l}}\right)
\end{aligned}
$$

where

$$
\vec{f}_{l}^{(k)}(u):=\frac{\vec{b}_{l}^{(k)}(u)}{\left(\lambda_{k}^{\prime}-l\right) !}, \quad l=1, \ldots, \lambda_{k}^{\prime} .
$$

In order to evaluate the terms with logarithm, we observe that for any function $g(\lambda)$ holomorphic along $L_{k}(\eta)$, including $\lambda=u_{k}$, we have

$$
\int_{\gamma_{k}(\eta)} g(\lambda) \ln \left(\lambda-u_{k}\right) \mathrm{d} \lambda=\int_{L_{k}(\eta)^{-}} g(\lambda) \ln \left(\lambda-u_{k}\right)_{-} \mathrm{d} \lambda-\int_{L_{k}(\eta)^{+}} g(\lambda) \ln \left(\lambda-u_{k}\right)_{+} \mathrm{d} \lambda
$$

where $L_{k}(\eta)^{+}$and $L_{k}(\eta)^{-}$, respectively, are the left and right parts of $L_{k}(\eta)$, oriented from 0 to $\infty$. Since $\ln \left(\lambda-u_{k}\right)_{+}=\ln \left(\lambda-u_{k}\right)_{+}-2 \pi i$, we conclude that

$$
\int_{\gamma_{k}(\eta)} g(\lambda) \ln \left(\lambda-u_{k}\right) \mathrm{d} \lambda=2 \pi i \int_{L_{k}(\eta)} g(\lambda) \mathrm{d} \lambda .
$$


Keeping into account that the integral along $\Gamma_{\alpha}$ can be interchanged with that along $\gamma_{k}$, it follows that

$$
\begin{aligned}
\frac{1}{2 \pi i} \int_{\Gamma_{\alpha}(\eta)} \vec{\Psi}_{k}(\lambda, u \mid v) \ln \left(\lambda-u_{k}\right) e^{z \lambda} \mathrm{d} \lambda & =\int_{L_{k}(\eta)} \vec{\Psi}_{k}(\lambda, u \mid v) e^{z \lambda} \mathrm{d} \lambda \\
& =\int_{L_{k}(\eta)} \sum_{l=0}^{\infty} \vec{d}_{l}^{(k)}(u)\left(\lambda-u_{k}\right)^{l} e^{z \lambda} \mathrm{d} \lambda
\end{aligned}
$$

We conclude, by the standard evaluation of the remainder analogous to $R_{\mathcal{N}}(z)$ considered before, and the variation of $\eta$ in the range $\left(\eta_{\nu+1}, \eta_{\nu}\right)$, that ${ }^{20}$

$$
\begin{aligned}
\int_{L_{k}(\eta)} \vec{\Psi}_{k}(\lambda, u \mid v) e^{z \lambda} \mathrm{d} \lambda & \sim e^{u_{k} z}\left(\sum_{l=0}^{\infty}(-1)^{l+1} l ! \vec{d}_{l}^{(k)}(u) z^{-l-1}\right), \quad z \rightarrow \infty \text { in } \widehat{\mathcal{S}}_{\nu} . \\
& =z^{\lambda_{k}^{\prime}} e^{u_{k} z}\left(\sum_{l=\lambda_{k}^{\prime}+1}^{\infty} \vec{f}_{l}^{(k)}(u) z^{-l}\right),
\end{aligned}
$$

where

$$
\vec{f}_{l}^{(k)}(u):=(-1)^{l-\lambda_{k}^{\prime}}\left(l-\lambda_{k}^{\prime}-1\right) ! \vec{d}_{l-\lambda_{k}^{\prime}-1}^{(k)}(u), \quad l \geq \lambda_{k}^{\prime}+1 .
$$

In conclusion, we have the expansion

$$
\vec{Y}_{k}(z, u \mid v) \sim z^{\lambda_{k}^{\prime}} e^{u_{k} z}\left(\vec{e}_{k}+\sum_{l=1}^{\infty} \vec{f}_{l}^{(k)}(u) z^{-l}\right), \quad z \rightarrow \infty \text { in } \widehat{\mathcal{S}}_{v}
$$

with coefficients $\vec{f}_{l}{ }^{(k)}(u)$ holomorphic in $\mathbb{D}\left(u^{c}\right)$ defined in (7.13)-(7.15). Notice that, in exceptional cases, $\vec{\Psi}_{k}$ may be identically zero, so that

$$
\vec{f}_{l}^{(k)}=0 \text { for } l \geq \lambda_{k}^{\prime}+1
$$

c) Case $\lambda_{k}^{\prime} \in \mathbb{Z}_{-}=\{-1,-2, \ldots\}$

- Construction of $\vec{Y}_{k}(z, u \mid v)$. Definition (7.5) is

$$
\vec{Y}_{k}(z, u \mid v):=\int_{L_{k}(\eta)} e^{\lambda z} \vec{\Psi}_{k}(\lambda, u \mid v) \mathrm{d} \lambda \equiv \int_{\mathcal{L}_{\alpha}(\eta)} e^{\lambda z} \vec{\Psi}_{k}(\lambda, u \mid v) \mathrm{d} \lambda .
$$

In the last equality, we have used the fact that $\vec{\Psi}_{k}(\lambda, u \mid v)$ is analytic in $\mathbb{D}_{\alpha} \times \mathbb{D}\left(u^{c}\right)$, where $\lambda_{\alpha}=u_{k}^{c}$.

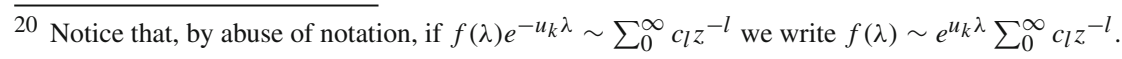


We conclude analogously to previous cases that $\vec{Y}_{k}(z, u \mid v)$ is analytic in $\widehat{\mathcal{S}}_{v} \times \mathbb{D}\left(u^{c}\right)$. It is a solution of (1.1), by (2.25), because $\vec{\Psi}_{k}(\lambda, u \mid \nu)$ is analytic at $\lambda=u_{k}$ and behaves as in (5.4)-(5.5), so that

$$
\begin{aligned}
\left.e^{\lambda z}(\lambda I-\Lambda(u)) \vec{\Psi}_{k}(\lambda, u \mid v)\right|_{\mathcal{L}_{\alpha}} & =\left.e^{\lambda z}(\lambda I-\Lambda(u)) \vec{\Psi}_{k}(\lambda, u \mid v)\right|_{L_{k}} \\
& =0-\left(u_{k} I-\Lambda(u)\right) \vec{\Psi}_{k}\left(\lambda, u_{k} \mid v\right)=0 .
\end{aligned}
$$

- Asymptotic behaviour. We have, from (5.4)-(5.5),

$$
\vec{Y}_{k}(z, u \mid v)=\int_{\mathcal{L}_{\alpha}(\eta)} e^{\lambda z}\left(\frac{(-1)^{\lambda_{k}^{\prime}} \vec{e}_{k}}{\left(-\lambda_{k}^{\prime}-1\right) !}\left(\lambda-u_{k}\right)^{-\lambda_{k}^{\prime}-1}+\sum_{l \geq 1} \vec{b}_{l}^{(k)}(u)\left(\lambda-u_{k}\right)^{l-\lambda_{k}^{\prime}-1}\right) \mathrm{d} \lambda
$$

We integrate term by term in order to obtain the asymptotic expansion (the remainder for the truncated series is evaluate in standard way, as $R_{\mathcal{N}}(z)$ above). For the integration, we use

$$
\begin{aligned}
& \int_{L_{k}(\eta)}\left(\lambda-u_{k}\right)^{m} e^{\lambda z} \mathrm{~d} \lambda=\frac{e^{u_{k} z}}{z^{m+1}} \int_{+\infty e^{i \phi}}^{0} x^{m} e^{x} \mathrm{~d} x=\frac{e^{u_{k} z}}{z^{m+1}} m !(-1)^{m+1} \\
& \frac{\pi}{2}<\phi<\frac{3 \pi}{2}
\end{aligned}
$$

We obtain, analogously to previous cases,

$$
\vec{Y}_{k}(z, u \mid v) \sim z^{\lambda_{k}^{\prime}} e^{u_{k} z}\left(\vec{e}_{k}+\sum_{l=1}^{\infty} \vec{f}_{l}^{(k)}(u) z^{-l}\right), \quad z \rightarrow \infty \text { in } \widehat{\mathcal{S}}_{\nu},
$$

where the holomorphic in $\mathbb{D}\left(u^{c}\right)$ coefficients are

$$
\vec{f}_{l}^{(k)}(u):=(-1)^{l-\lambda_{k}^{\prime}}\left(l-\lambda_{k}^{\prime}-1\right) ! \vec{b}_{l}^{(k)}(u)
$$

Remark 7.2 We cannot use $\vec{\Psi}_{k}^{(\text {sing) }}(\lambda, u \mid v)$ in (5.8) to define $\vec{Y}_{k}(z, u \mid v)$ if $u$ varies in the whole $\mathbb{D}\left(u^{c}\right)$. On the other hand, if $u$ is restricted to a $\tau$-cell, so that the eigenvalues $u_{j}$ are all distinct, by (7.14) we can write

$$
\vec{Y}_{k}(z, u \mid v)=\int_{L_{k}(\eta)} e^{\lambda z} \vec{\Psi}_{k}(\lambda, u \mid v) \mathrm{d} \lambda \underset{(7.14)}{=} \frac{1}{2 \pi i} \int_{\gamma_{k}(u)} e^{\lambda z} \vec{\Psi}_{k}(\lambda, u \mid v) \ln \left(\lambda-u_{k}\right) \mathrm{d} \lambda .
$$

Then, we can use the local expansion (5.9) and the fact that $\int_{\gamma_{k}(u)} \operatorname{reg}\left(\lambda-u_{k}\right) \mathrm{d} \lambda=0$, receiving

$$
\vec{Y}_{k}(z, u \mid \nu)=\frac{1}{2 \pi i} \int_{\gamma_{k}(u)} e^{\lambda z} \vec{\Psi}_{k}^{(\text {sing })}(\lambda, u \mid \nu) \mathrm{d} \lambda
$$




\section{Fundamental matrix solutions}

The vector solutions $\vec{Y}_{k}(z, u \mid v)$ constructed above can be arranged as columns of the matrix

$$
Y_{\nu}(z, u):=\left[\vec{Y}_{k}(z, u \mid v)|\cdots| \vec{Y}_{n}(z, u \mid v)\right]
$$

which thus solves system (1.1). From the general theory of differential systems, it admits analytic continuation as analytic matrix valued function on $\mathcal{R}(\mathbb{C} \backslash\{0\}) \times \mathbb{D}\left(u^{c}\right)$. Letting $B=\operatorname{diag} A=\operatorname{diag}\left(\lambda_{1}^{\prime}, \ldots, \lambda_{n}^{\prime}\right)$, the asymptotic expansions obtained above are summarized as

$$
\begin{gathered}
Y_{v}(z, u \mid v) z^{-B} e^{-\Lambda(u) z} \sim F(z, u)=I+\sum_{l=1}^{\infty} F_{l}(u) z^{-l}, \quad z \rightarrow \infty \text { in } \widehat{\mathcal{S}}_{v}, \\
F_{l}(u)=\left[\vec{f}_{l}^{(1)}(u)|\cdots| \vec{f}_{l}^{(n)}(u)\right] .
\end{gathered}
$$

Therefore, the coefficients $F_{l}(u)$ of the formal solution $Y_{F}(z, u)=F(z, u) z^{B} e^{\Lambda(u) z}$ are holomorphic in $\mathbb{D}\left(u^{c}\right)$. Moreover, the leading term is the identity $I$, which implies that $Y_{v}(z, u)$ is a fundamental matrix solution.

Consider now another direction $\eta$, satisfying $\eta_{\nu+\mu+1}<\eta<\eta_{\nu+\mu}$. The above discussion can be repeated. We obtain a fundamental matrix solution $Y_{\nu+\mu}(z, u)$ with canonical asymptotics $Y_{F}(z, u)$ in $\widehat{S}_{\nu+\mu}$. Again, for $\eta$ satisfying $\eta_{\nu+2 \mu+1}<\eta<$ $\eta_{\nu+2 \mu}$ we obtain the analogous result for $Y_{\nu+2 \mu}(z, u)$ with canonical asymptotics in $\widehat{S}_{\nu+2 \mu}$. This can be repeated for every $\nu+h \mu, h \in \mathbb{Z}$, obtaining the fundamental matrix solutions $Y_{v+h \mu}(z, u)$ with canonical asymptotics $Y_{F}(z, u)$ in $\widehat{S}_{v+h \mu}$. So, Points (1) and (2) of Theorem 7.1 are proved.

Stokes matrices are defined by (7.7). Thus, $\mathbb{S}_{v+h \mu}(u)=Y_{v+h \mu}(z, u)^{-1} Y_{v+(h+1) \mu}$ $(z, u)$ is holomorphic in $\mathbb{D}\left(u^{c}\right)$. Let us consider the relations for $h=0,1$ :

$$
Y_{\nu+\mu}(z, u)=Y_{\nu}(z, u) \mathbb{S}_{\nu}(u), \quad Y_{\nu+2 \mu}(z, u)=Y_{\nu+\mu}(z, u) \mathbb{S}_{\nu+\mu}(u) .
$$

Let $u$ be fixed in a $\tau$-cell, so that $\Lambda$ has distinct eigenvalues. From Theorem 2.3 at the fixed $u$ we receive

$$
\begin{aligned}
\left(\mathbb{S}_{\nu}(u)\right)_{j k}=\left\{\begin{array}{cl}
e^{2 \pi i \lambda_{k}^{\prime}} \alpha_{k} c_{j k}^{(v)} & \text { for } j \prec k, \\
1 & \text { for } j=k, \\
0 & \text { for } j \succ k,
\end{array},\right. \\
\left(\mathbb{S}_{\nu+\mu}^{-1}(u)\right)_{j k}=\left\{\begin{array}{cc}
0 & \text { for } j \prec k, \\
1 & \text { for } j=k, \\
-e^{2 \pi i\left(\lambda_{k}^{\prime}-\lambda_{j}^{\prime}\right)} \alpha_{k} c_{j k}^{(v)} & \text { for } j \succ k .
\end{array}\right.
\end{aligned}
$$


Here, for $j \neq k$ the ordering relation $\left.j \prec k \Longleftrightarrow \Re\left(z\left(u_{j}-u_{k}\right)\right)\right|_{\arg z=\tau<0 \text { is well }}$ defined for every $u$ in the $\tau$-cell, because no Stokes rays $\Re\left(z\left(u_{j}-u_{k}\right)\right)=0$ cross $\arg z=\tau$ as $u$ varies in the $\tau$-cell.

The relation $j \prec k$ may change to $j \succ k$ when passing from one $\tau$-cell to another only for a pair $u_{j}, u_{k}$ such that $u_{j}^{c}=u_{k}^{c}$. This is due to the choice of $\epsilon_{0}$ as in (5.1). On the other hand, $c_{j k}^{(v)}=0$ whenever $u_{j}^{c}=u_{k}^{c}$. This means that (7.9) is true at every fixed $u$ in every $\tau$-cell, with ordering relation $j \prec k$ defined for $j \neq k$ such that $u_{j}^{c} \neq u_{k}^{c}$, namely $\mathfrak{R}\left(z\left(u_{j}^{c}-u_{k}^{c}\right)\right)<0$ when $\arg z=\tau$.

Since the $\mathbb{S}_{\nu+h \mu}$ are holomorphic in $\mathbb{D}\left(u^{c}\right)$ and the $c_{j k}^{(v)}$ are constant in $\mathbb{D}\left(u^{c}\right)$, we conclude that Stokes matrices are constant in $\mathbb{D}\left(u^{c}\right)$ and hence (7.9) holds in $\mathbb{D}\left(u^{c}\right)$. The vanishing conditions (7.8) follow from the vanishing conditions (5.14) for the connection coefficients, plus the fact that we can generate all the $\mathbb{S}_{\nu+h \mu}$ from the formula $\mathbb{S}_{\nu+2 \mu}=e^{-2 \pi i B} \mathbb{S}_{\nu} e^{2 \pi i B}$.

\section{Non-uniqueness at $u=u^{c}$ of the formal solution}

By Laplace transform, we prove Corollary 2.1 in Background 1, asserting that system (2.19) has unique formal solution if and only if the constant diagonal entries of $A(u)$ satisfy the partial non-resonance

$$
\lambda_{i}^{\prime}-\lambda_{j}^{\prime} \notin \mathbb{Z} \backslash\{0\} \quad \text { for every } i \neq j \text { such that } u_{i}^{c}=u_{j}^{c}
$$

Otherwise, the Laplace transform will be proved to generate a family of formal solutions

$$
\stackrel{\circ}{Y}_{F}(z)=\left(I+\sum_{l=1}^{\infty} \stackrel{\circ}{F}_{l} z^{-l}\right) z^{B} e^{\Lambda\left(u^{c}\right) z}
$$

whose coefficients $\stackrel{\circ}{F}_{l}$ depend on a finite number of arbitrary parameters.

Due to the strategy of Sect. 6.6, it will suffice to consider the generic case when all $\lambda_{1}^{\prime}, \ldots, \lambda_{n}^{\prime} \notin \mathbb{Z}$ and $A$ has no integer eigenvalues. Indeed, if this is not the case, the gauge transformation (6.46) relates a formal solution ${ }_{\gamma} Y_{F}$ to $Y_{F}$ at any point $u$, through (6.48), so that the coefficients $F_{l}$ of a formal expansion do not depend on $\gamma$. We are interested in these coefficients.

Consider system (1.4) under the assumptions that it is (strongly) isomonodromic in $\mathbb{D}\left(u^{c}\right)$, so that $(A)_{i j}\left(u^{c}\right)=0$ for $u_{i}^{c}=u_{j}^{c}$. For simplicity, we order the eigenvalues as in (6.1)-(6.2). Since $B_{1}(u), \ldots, B_{n}(u)$ are holomorphic at $u^{c}$, system (1.4) at $u=u^{c}$ is

$$
\frac{\mathrm{d} \Psi}{\mathrm{d} \lambda}=\left(\frac{\sum_{j=1}^{p_{1}} B_{j}\left(u^{c}\right)}{\lambda-\lambda_{1}}+\frac{\sum_{j=p_{1}+1}^{p_{1}+p_{2}} B_{j}\left(u^{c}\right)}{\lambda-\lambda_{2}}+\cdots+\frac{\sum_{j=p_{1}+\cdots+p_{s-1}+1}^{n} B_{j}\left(u^{c}\right)}{\lambda-\lambda_{s}}\right) \Psi
$$


Let $G^{\left(p_{1}\right)}$ be as in (6.24). The gauge transformation $\Psi(\lambda)=G^{\left(p_{1}\right)} \widetilde{\Psi}(\lambda)$ yields

$$
\frac{\mathrm{d} \widetilde{\Psi}}{\mathrm{d} \lambda}=\left(\frac{T^{\left(p_{1}\right)}}{\lambda-\lambda_{1}}+\sum_{\alpha=2}^{s} \frac{D_{\alpha}^{\left(p_{1}\right)}}{\lambda-\lambda_{\alpha}}\right) \widetilde{\Psi}
$$

where

$$
T^{\left(p_{1}\right)}:=T^{(1)}+\cdots+T^{\left(p_{1}\right)}=\operatorname{diag}(-\lambda_{1}^{\prime}-1, \ldots,-\lambda_{p_{1}}^{\prime}-1, \underbrace{0, \ldots, 0}_{n-p_{1}}) .
$$

and $D_{\alpha}^{\left(\boldsymbol{p}_{1}\right)}:=G^{\left(\boldsymbol{p}_{1}\right)^{-1}} \cdot \sum_{j=p_{1}+\cdots+p_{\alpha-1}+1}^{p_{1}+\cdots+p_{\alpha}} B_{j}\left(u^{c}\right) \cdot G^{\left(\boldsymbol{p}_{1}\right)}$. The matrix coefficient in system (8.2) has convergent Taylor series at $\lambda=\lambda_{1}$

$$
\frac{\mathrm{d} \widetilde{\Psi}}{\mathrm{d} \lambda}=\frac{1}{\lambda-\lambda_{1}}\left(T^{\left(\boldsymbol{p}_{1}\right)}+\sum_{m=1}^{\infty} \mathfrak{D}_{m}\left(\lambda-\lambda_{1}\right)^{m}\right) \widetilde{\Psi}, \quad \mathfrak{D}_{m}=\sum_{\alpha=2}^{s} \frac{(-1)^{m+1}}{\left(\lambda_{1}-\lambda_{\alpha}\right)^{m}} D_{\alpha}^{\left(\boldsymbol{p}_{1}\right)}
$$

We consider $\eta_{\nu+1}<\eta<\eta_{\nu}$ and $\lambda$ in the plane with branch cuts $\mathcal{L}_{\alpha}=\mathcal{L}_{\alpha}(\eta)$ issuing from $\lambda_{1}, \ldots, \lambda_{s}$ to infinity in direction $\eta$, as in (5.2). Close to the Fuchsian singularity $\lambda=\lambda_{1}$ a fundamental matrix solution to (8.1) has Levelt form

$$
\stackrel{\circ}{\Psi}^{\left(p_{1}\right)}(\lambda)=G^{\left(p_{1}\right)}\left(I+\sum_{l=1}^{\infty} \mathfrak{G}_{l}\left(\lambda-\lambda_{1}\right)^{l}\right)\left(\lambda-\lambda_{1}\right)^{T^{\left(p_{1}\right)}}
$$

where the matrix entries $\left(\mathfrak{G}_{l}\right)_{i j}, 1 \leq i \leq j \leq n$, are recursively computed by the following formulae (see Appendix $\mathrm{C}$ for an explanation of $(8.3)$, or [27,62]).

- If $T_{i i}^{\left(p_{1}\right)}-T_{j j}^{\left(p_{1}\right)}=l$ positive integer, $\left(\mathfrak{G}_{l}\right)_{i j}$ is arbitrary.

- If $T_{i i}^{\left(\boldsymbol{p}_{1}\right)}-T_{j j}^{\left(\boldsymbol{p}_{1}\right)} \neq l$ (positive integer)

$$
\left(\mathfrak{G}_{l}\right)_{i j}=\frac{1}{T_{j j}^{\left(\boldsymbol{p}_{1}\right)}-T_{i i}^{\left(\boldsymbol{p}_{1}\right)}+l}\left(\sum_{p=1}^{l-1} \mathfrak{D}_{l-p} \mathfrak{G}_{l}+\mathfrak{D}_{l}\right)_{i j} \quad(\text { sum is zero for } l=1) .
$$

Since we have assumed that all the $\lambda_{k}^{\prime}$ are not integers, the only possibility to have $T_{i i}^{\left(\boldsymbol{p}_{1}\right)}-T_{j j}^{\left(\boldsymbol{p}_{1}\right)}=l$ occurs for $1 \leq i, j \leq p_{1}$, precisely

$$
T_{i i}^{\left(\boldsymbol{p}_{1}\right)}-T_{j j}^{\left(\boldsymbol{p}_{1}\right)}=\lambda_{j}^{\prime}-\lambda_{i}^{\prime}=l
$$

In this case, (8.3) is a family depending on a finite number of parameters due to the arbitrary $\left(\mathfrak{G}_{l}\right)_{i j}$. Thus, in the first $p_{1}$ columns of a solution of type (8.3)

$$
\stackrel{\stackrel{\circ}{\Psi}}{j}_{j}(\lambda \mid v)=\left(\Gamma\left(\lambda_{k}^{\prime}+1\right) \vec{e}_{k}+\sum_{l=1}^{\infty} \stackrel{\circ}{b}_{l}^{(j)}\left(\lambda-\lambda_{1}\right)\right)\left(\lambda-\lambda_{1}\right)^{-\lambda_{j}^{\prime}-1}, \quad j=1, \ldots, p_{1} .
$$


the vectors $\dot{b}_{l}^{(j)}$ contain a finite number of parameters. The Laplace transform

$$
\overrightarrow{\stackrel{\circ}{Y}}_{j}(z \mid \nu)=\int_{\Gamma_{1}(\eta)} e^{z \lambda} \overrightarrow{\stackrel{\circ}{\Psi}}_{j}(\lambda \mid \nu) \mathrm{d} \lambda, \quad j=1, \ldots, p_{1},
$$

yields the first $p_{1}$ columns of a fundamental matrix solution of (2.19). Repeating the same computations of Section 7 , we obtain, for $j=1, \ldots, p_{1}$,

$$
\overrightarrow{\stackrel{\circ}{Y}}_{j}(z \mid v) z^{-\lambda_{j}^{\prime}} e^{-\lambda_{1} z} \sim \vec{e}_{j}+\sum_{l=1}^{\infty} \frac{\stackrel{\circ}{b}_{l}^{(j)}}{\Gamma\left(\lambda_{j}^{\prime}+1-l\right)} \frac{1}{z^{l}}, \quad z \rightarrow \infty \text { in } \mathcal{S}_{v},
$$

where $\mathcal{S}_{v}$ is given in (7.1). We repeat the same construction at all $\lambda_{1}, \ldots, \lambda_{s}$. This yields a family of fundamental matrix solutions of (2.19)

$$
\stackrel{\circ}{Y}_{v}(z)=\left[\stackrel{\vec{\circ}}{Y}_{1}(z \mid v)|\cdots| \overrightarrow{\stackrel{\circ}{Y}}_{n}(z \mid v)\right]
$$

depending on a finite number of parameters, with the behaviour for $z \rightarrow \infty$ in $S_{v}$

$$
\begin{aligned}
\stackrel{\circ}{Y}_{v}(z) \sim \stackrel{\circ}{Y}_{F}(z) & =\left(I+\sum_{l=1}^{\infty} \stackrel{\circ}{F}_{l} z^{-l}\right) z^{B} e^{\Lambda\left(u^{c}\right) z} ; \quad \stackrel{\circ}{F}_{l}=\left[{\left.\stackrel{\circ}{\dot{f}_{1}^{(l)}}|\cdots| \vec{\circ}_{n}^{(l)}\right],}_{\vec{\circ}_{\dot{\circ}}^{(l)}}=\frac{\vec{\circ}_{j}^{(l)}}{\Gamma\left(\lambda_{j}^{\prime}+1-l\right)} .\right.
\end{aligned}
$$

We conclude that the formal solution is not unique whenever a condition (8.4) occurs. Only one element in the family satisfies $\stackrel{\circ}{Y}_{F}(z)=Y_{F}\left(z, u^{c}\right)$.

Remark 8.1 If we choose one formal solution $\stackrel{\circ}{Y}_{F}(z)$, then the corresponding $\stackrel{\circ}{Y}_{v}(z)$ with asymptotic expansion $\stackrel{\circ}{Y}_{F}(z)$ in $\mathcal{S}_{v}$ is unique. For more details on the Stokes phenomenon at $u=u^{c}$, see [13].

Acknowledgements I remember with gratitude professor B. Dubrovin, who gave us the initial motivation to investigate the problem of non-generic isomonodromic deformations, contributing in our discussions with insight, experience and enthusiasm. I would like to thank professor $\mathrm{H}$. Iritani for kindly drawing my attention to the proof of proposition 2.5.1 of [22], and professor C. Sabbah for his clear explanation of [52,60] given at a talk I attended, which allowed me to appreciate the importance of the geometric approach. I also thank doctor G. Cotti for several stimulating discussions in the background of this paper. Finally, I would like to thank very much the anonymous referees for valuable comments, suggestions and corrections that have improved the paper. The author is a member of the European Union's H2020 research and innovation programme under the Marie Skłlodowska-Curie grant No. 778010 IPaDEGAN.

Funding Open access funding provided by Scuola Internazionale Superiore di Studi Avanzati - SISSA within the CRUI-CARE Agreement.

\section{Declaration}

Conflict of interest The corresponding author states that there is no conflict of interest. 
Open Access This article is licensed under a Creative Commons Attribution 4.0 International License, which permits use, sharing, adaptation, distribution and reproduction in any medium or format, as long as you give appropriate credit to the original author(s) and the source, provide a link to the Creative Commons licence, and indicate if changes were made. The images or other third party material in this article are included in the article's Creative Commons licence, unless indicated otherwise in a credit line to the material. If material is not included in the article's Creative Commons licence and your intended use is not permitted by statutory regulation or exceeds the permitted use, you will need to obtain permission directly from the copyright holder. To view a copy of this licence, visit http://creativecommons.org/licenses/by/4.0/.

\section{Appendix A. non-normalized Schlesinger system}

Lemma 9.1 The integrability condition $d P=P \wedge P$ of the Pfaffian system (3.2) defined on a polydisc $\mathbb{D}\left(u^{0}\right)$ contained in a $\tau$-cell is the non-normalized Schlesinger system (3.3)-(3.5).

Proof For every $i \in\{1, \ldots, n\}$, the Pfaffian system (3.2) can be rewritten as

$$
\begin{aligned}
P= & \left(\frac{B_{i}}{\lambda-u_{i}}+\sum_{j \neq i} \frac{B_{j}}{\lambda-u_{j}}\right) d\left(\lambda-u_{i}\right)+\sum_{j \neq i}\left(\gamma_{j}-\frac{B_{j}}{\lambda-u_{j}}\right) d\left(u_{j}-u_{i}\right) \\
& +\sum_{j=1}^{n} \gamma_{j}(u) \mathrm{d} \lambda .
\end{aligned}
$$

We study $\lambda-u_{i} \rightarrow 0$, while $u_{j}-u_{i} \neq 0$ for $j \neq i$ in $\mathbb{D}\left(u^{0}\right)$. In new variables

$$
\lambda=\lambda, \quad y_{i}=\lambda-u_{i}, \quad y_{j}=u_{j}-u_{i}, \quad j \neq i,
$$

$P$ is rewritten in the following way (which defines the matrices $\mathcal{A}_{j}(y)$ )

$$
\begin{aligned}
P= & \left(\frac{B_{i}}{y_{i}}+\sum_{j \neq i} \frac{B_{j}}{y_{i}-y_{j}}\right) \mathrm{d} y_{i}+\sum_{j \neq i}\left(\gamma_{j}-\frac{B_{j}}{y_{i}-y_{j}}\right) \mathrm{d} y_{j}+\sum_{j=1}^{n} \gamma_{j}(y) \mathrm{d} \lambda \\
& =: \mathcal{A}_{i}(y) \mathrm{d} y_{i}+\sum_{j \neq i} \mathcal{A}_{j}(y) \mathrm{d} y_{j}+\sum_{j=1}^{n} \gamma_{j}(y) \mathrm{d} \lambda .
\end{aligned}
$$

The only singular term at $y_{i}=0$ is $B_{i} / y_{i}$ in $\mathcal{A}_{i}(y)$. The components relative to $\mathrm{d} y_{1}, \ldots, \mathrm{d} y_{n}$ of $d P=P \wedge P$ are

$$
\frac{\partial \mathcal{A}_{l}}{\partial y_{k}}+\mathcal{A}_{l} \mathcal{A}_{k}=\frac{\partial \mathcal{A}_{k}}{\partial y_{l}}+\mathcal{A}_{k} \mathcal{A}_{l}, \quad k \neq l,
$$

For $k \neq i$ and $l=i$, from (9.1), we receive

$$
\frac{\partial}{\partial y_{k}}\left(\frac{B_{i}}{y_{i}}+\operatorname{reg}\left(y_{i}\right)\right)+\left(\frac{B_{i}}{y_{i}}+\operatorname{reg}\left(y_{i}\right)\right) \mathcal{A}_{k}=\frac{\partial \mathcal{A}_{k}}{\partial y_{i}}+\mathcal{A}_{k}\left(\frac{B_{i}}{y_{i}}+\operatorname{reg}\left(y_{i}\right)\right),
$$


where reg $\left(y_{i}\right)$ stands for an analytic term at $y_{i}=0$. We expand the above in Taylor series at $y_{i}=0$. The singular term (the residue at $y_{i}=0$ ) is

$$
\frac{\partial B_{i}}{\partial y_{k}}=\left[\left.\mathcal{A}_{k}\right|_{y_{i}=0}, B_{i}\right]=\frac{\left[B_{k}, B_{i}\right]}{u_{k}-u_{i}}+\left[\gamma_{k}, B_{i}\right], \quad k \neq i .
$$

The above gives the non-normalized Schlesinger Eqs. (3.4)-(3.5), because

$$
\begin{aligned}
\frac{\partial B_{i}}{\partial y_{k}} & =\frac{\partial B_{i}}{\partial\left(u_{k}-u_{i}\right)}=\frac{\partial u_{k}}{\partial\left(u_{k}-u_{i}\right)} \frac{\partial B_{i}}{\partial u_{k}}=\frac{\partial B_{i}}{\partial u_{k}}, \\
\frac{\partial B_{i}}{\partial u_{i}} & =\sum_{k \neq i} \frac{\partial\left(u_{k}-u_{i}\right)}{\partial u_{i}} \frac{\partial B_{i}}{\partial\left(u_{k}-u_{i}\right)}=-\sum_{k \neq i} \frac{\partial B_{i}}{\partial u_{k}} \Longrightarrow \sum_{k=1}^{n} \frac{\partial B_{i}}{\partial u_{k}}=0 .
\end{aligned}
$$

If we write the components of $d P=P \wedge P$ referring to $\mathrm{d} y_{l}$ ad $\mathrm{d} \lambda$, and we substitute into them (9.3)-(9.4), we receive (3.3), namely $\partial_{l} \gamma_{k}-\partial_{k} \gamma_{l}=\gamma_{l} \gamma_{k}-\gamma_{k} \gamma_{l}$.

Corollary 9.1 A solution $B_{i}(u), i=1, \ldots, n$, of (3.3)-(3.5) is holomorphically similar to a constant Jordan form on $\mathbb{D}\left(u^{0}\right)$. The similarity is realized by a fundamental matrix solution $G^{(i)}(u)$ of the Pfaffian system (9.6).

Proof We must show that there exists a holomorphically invertible $G^{(i)}(u)$ on $\mathbb{D}\left(u^{0}\right)$ such that $\left(G^{(i)}\right)^{-1} B_{i} G^{(i)}$ is a constant Jordan form. The conditions (9.1) for $k, l \neq i$ can be evaluated at $y_{i}=0$, and become

$$
\frac{\left.\partial \mathcal{A}_{l}\right|_{y_{i}=0}}{\partial y_{k}}+\left.\left.\mathcal{A}_{l}\right|_{y_{i}=0} \mathcal{A}_{k}\right|_{y_{i}=0}=\frac{\left.\partial \mathcal{A}_{k}\right|_{y_{i}=0}}{\partial y_{l}}+\left.\left.\mathcal{A}_{k}\right|_{y_{i}=0} \mathcal{A}_{l}\right|_{y_{i}=0}, \quad k \neq i, l \neq i, k \neq l .
$$

Hence, the following Pfaffian system is Frobenius integrable

$$
\frac{\partial G}{\partial y_{k}}=\left.\mathcal{A}_{k}\right|_{y_{i}=0} G \equiv\left(\frac{B_{k}}{u_{k}-u_{i}}+\gamma_{k}\right) G, \quad k \neq i .
$$

Using the chain rule as in (9.3), we receive (6.8)

$$
\frac{\partial G}{\partial u_{k}}=\left(\frac{B_{k}}{u_{k}-u_{i}}+\gamma_{k}\right) G, \quad k \neq i, \quad \frac{\partial G}{\partial u_{i}}=-\sum_{k \neq i}\left(\frac{B_{k}}{u_{k}-u_{i}}+\gamma_{k}\right) G
$$

Notice that for both $\varphi(u)=B_{i}(u)$ and $\varphi(u)=G(u)$ we have

$$
\sum_{k=1}^{n} \frac{\partial \varphi}{\partial u_{k}}=0 \Longrightarrow \varphi(u)=\varphi\left(u_{1}-u_{i}, \ldots, u_{n}-u_{i}\right)
$$

We can take a solution $G(u)$ which holomorphically reduces $B_{i}$ to Jordan form. Indeed

$$
\text { for } k \neq i, \quad \frac{\partial}{\partial y_{k}}\left(G^{-1} B_{i} G\right)=-G^{-1} \frac{\partial G}{\partial y_{k}} G^{-1} B_{i} G+G^{-1} \frac{\partial B_{i}}{\partial y_{k}} G+G^{-1} B_{i} \frac{\partial G}{\partial y_{k}}
$$




$$
\begin{aligned}
& \underset{(9.2),(9.5)}{=}-\left.G^{-1} \mathcal{A}_{k}\right|_{y_{i}=0} B_{i} G \\
& \quad+G^{-1}\left[\left.\mathcal{A}_{k}\right|_{y_{i}=0}, B_{i}\right] G+\left.G^{-1} B_{i} \mathcal{A}_{k}\right|_{y_{i}=0} G \\
& \quad=0 .
\end{aligned}
$$

Therefore, keeping into account (9.7), we see that $\left.\mathcal{B}_{i}:=G^{-1}(u) B_{i}(u) G(u)\right)$ is independent of $u$. Thus, there exists a constant matrix $\mathcal{G}$ such that $\mathcal{G}^{-1} \mathcal{B}_{i} \mathcal{G}$ is a constant Jordan form, and $G^{(i)}(u):=G(u) \mathcal{G}$ realizes the holomorphic "Jordanization". The above arguments are standard, see for example [28].

If the $B_{i}(u)$ are holomorphic on $\mathbb{D}\left(u^{c}\right)$ and the vanishing conditions (4.1) hold, the coefficients of the Pfaffian system (6.39) are holomorphic on $\mathbb{D}\left(u^{c}\right)$, so that $G^{(i)}(u)$ extends holomorphically there, and Corollary 9.1 holds on $\mathbb{D}\left(u^{c}\right)$.

\section{Appendix B. Proof of Proposition 3.1}

According to Theorem 2.1, system (1.1) is strongly isomonodromic in $\mathbb{D}\left(u^{0}\right)$ contained in a $\tau$-cell of $\mathbb{D}\left(u^{c}\right)$ if and only if (3.1) holds. In this case $G^{(0)}$ in (2.12) holomorphically reduces $A(u)$ to constant Jordan form and satisfies

$$
d G^{(0)}=\sum_{j=1}^{n} \omega_{j}(u) \mathrm{d} u_{j} G^{(0)} .
$$

Proof of Proposition 3.1 Suppose that (1.1) is strongly isomonodromic, so that (3.1) holds. Let $\mathcal{A}:=-A-I$, so that $E_{k} \mathcal{A}=B_{k}$, and (3.1) are rewritten as $\partial_{i} \mathcal{A}=$ $\left[\omega_{i}(u), \mathcal{A}\right]$. We multiply these equations to the left by $E_{k}$, with $k \neq i$. We receive

$$
E_{k} \partial_{i} \mathcal{A}=E_{k}\left[\omega_{i}(u), \mathcal{A}\right]
$$

The 1.h.s. is $E_{k} \partial_{i} \mathcal{A}=\partial_{i} B_{k}$. The r.h.s. is

$$
E_{k}\left[\omega_{i}, \mathcal{A}\right]=E_{k} \omega_{i} \mathcal{A}-E_{k} \mathcal{A} \omega_{i}=E_{k} \omega_{i} \mathcal{A}-B_{k} \omega_{i}=\left(E_{k} \omega_{i} \mathcal{A}-\omega_{i} B_{k}\right)+\left[\omega_{i}, B_{k}\right]
$$

In conclusion

$$
\partial_{i} B_{k}=\left(E_{k} \omega_{i} \mathcal{A}-\omega_{i} B_{k}\right)+\left[\omega_{i}, B_{k}\right], \quad i \neq k
$$

The only terms we need to evaluate are

$$
\begin{aligned}
& E_{k} \omega_{i} \mathcal{A}-\omega_{i} B_{k}=E_{k}\left[F_{1}, E_{i}\right] \mathcal{A}-\left[F_{1}, E_{i}\right] B_{k} \\
& \quad=E_{k} F_{1} E_{i} \mathcal{A}+E_{i} F_{1} B_{k}=E_{k} F_{1} E_{i} B_{i}+E_{i} F_{1} E_{k} B_{k} .
\end{aligned}
$$

In the second line we have used $E_{i} E_{k}=E_{k} E_{i}=E_{i} B_{k}=0$, for $i \neq k$, and $E_{i}^{2}=E_{i}$. Now, observe that $E_{k} F_{1} E_{i}$ has zero entries, except for the entry $(k, i)$, 
which is $\left(F_{1}\right)_{k i}=(A)_{k i} /\left(u_{i}-u_{k}\right)$. This implies that

$$
E_{k} F_{1} E_{i} B_{i}+E_{i} F_{1} E_{k} B_{k}=\frac{\left[B_{i}, B_{k}\right]}{u_{i}-u_{k}} .
$$

In conclusion, we have proved that (3.1) implies (3.4). On the other hand (3.4)-(3.5) are equivalent to the system given by (3.4) and the equations

$$
\partial_{i} \sum_{k} B_{k}=\left[\omega_{i}, \sum_{k} B_{k}\right], \quad i=1, \ldots, n .
$$

which are exactly (3.1) if $B_{k}=E_{k} \mathcal{A}$. Finally, notice that (3.3), here with $\gamma_{j}=\omega_{j}$, is the integrability condition on $\mathbb{D}\left(u^{0}\right)$ of $d G=\sum_{j=1}^{n} \omega_{j}(u) \mathrm{d} u_{j} G$. On the other hand, it is a computation to see that (3.1) implies the the same conditions.

Conversely, let system (1.4) be strongly isomonodromic, so that the integrability conditions (3.3)-(3.5) hold. Firstly, we show that (3.4)-(3.5) imply a Pfaffian system for $A$ of type (3.1). To this end, we sum (3.4) and (3.5):

$$
\sum_{k=1}^{n} \partial_{i} B_{k}=\sum_{k \neq i} \frac{\left[B_{i}, B_{k}\right]}{u_{i}-u_{k}}-\sum_{k \neq i} \frac{\left[B_{i}, B_{k}\right]}{u_{i}-u_{k}}+\left[\gamma_{i}, \sum_{k=1}^{n} B_{k}\right]=\left[\gamma_{i}, \sum_{k=1}^{n} B_{k}\right] .
$$

Using $B_{k}=-E_{k}(A+I)$ and $\sum_{k} E_{k}=I$, the above becomes

$$
\partial_{i} A=\left[\gamma_{i}, A\right], \quad i=1, \ldots, n .
$$

Since $\gamma_{1}, \ldots, \gamma_{n}$ satisfy (3.3), it is directly verified that (10.2) is Frobenius integrable. Secondly, we must show that we can choose

$$
\gamma_{j}:=\omega_{j}=\left(\frac{A_{a b}\left(\delta_{a j}-\delta_{b j}\right)}{u_{a}-u_{b}}\right)_{a, b=1}^{n} \quad \text { as in (2.18). }
$$

Substituting this choice into (3.3), we see that if (10.2) holds, in the form $\partial_{i} A=$ $\left[\omega_{i}, A\right]$, then (3.3) are satisfied. ${ }^{21}$ Now, since (10.2) follows from (3.4) to (3.5) with matrices $B_{k}=-E_{k}(A+I)$, we conclude that (3.4)-(3.5) and the choice $\gamma_{j}=\omega_{j}$ guarantee that both (3.3) and (3.1) are satisfied.

The Schlesinger system can be used to show that there is a fundamental matrix solution $G^{(0)}$ of (10.1) that holomorphically reduces $A$ to constant Jordan form on $\mathbb{D}\left(u^{0}\right)$. Since $(3.3)$ is the integrability condition on $\mathbb{D}\left(u^{0}\right)$ of the linear Pfaffian system

$$
d G=\sum_{j=1}^{n} \omega_{j}(u) \mathrm{d} u_{j} G
$$

\footnotetext{
21 This is exactly what has been said before: (3.1) implies by computation the integrability conditions of (10.1), namely exactly Eq. (3.3) with $\gamma_{j}=\omega_{j}$.
} 
the latter admits holomorphic fundamental matrix solutions in $\mathbb{D}\left(u^{0}\right)$. Let $G(u)$ be one of them and define

$$
\widehat{B}_{k}:=G(u)^{-1} B_{k} G(u) .
$$

By direct computation, using (10.3) and its integrability (3.3), it is verified that (3.4)(3.5) (with $\gamma_{j}=\omega_{j}$ ) are equivalent to the normalized Schlesinger equations for the matrices $\widehat{B}_{k}$,

$$
\partial_{i} \widehat{B}_{k}=\frac{\left[\widehat{B}_{i}, \widehat{B}_{k}\right]}{u_{i}-u_{k}}, \quad i \neq k ; \quad \partial_{i} \widehat{B}_{i}=-\sum_{k \neq i} \frac{\left[\widehat{B}_{i}, \widehat{B}_{k}\right]}{u_{i}-u_{k}} .
$$

The above equations imply that

$$
\forall i=1, \ldots, n, \quad \partial_{i} \widehat{B}_{\infty}=0, \quad \text { where } \widehat{B}_{\infty}:=-\sum_{k=1}^{n} \widehat{B}_{k}
$$

Being $\widehat{B}_{\infty}$ constant, it can be put in constant Jordan form by a constant invertible matrix $P$, say $-\mathcal{J}=P^{-1} \widehat{B}_{\infty} P$. Since also $G(u) P$ solves (10.3), we can choose from the beginning $G(u)$ such that

$$
G^{-1}(u)\left(\sum_{k=1}^{n} B_{k}(u)\right) G(u)=\mathcal{J} \quad \text { constant Jordan form. }
$$

Now, observe that $\sum_{k=1}^{n} E_{k}=I$, so that

$$
\sum_{k=1}^{n} B_{k}=-\sum_{k=1}^{n} E_{k}(A+I)=-A-I .
$$

Thus, $G(u)$ also puts $A$ in constant Jordan form, so that ${ }^{22}$

$$
G(u)=G^{(0)}(u), \quad \text { where } G^{(0)} \text { is in (2.12). }
$$

In particular, $G^{(0)}$ satisfies (10.1).

The second part of the statement of Proposition 2.3 (Prop. 19.2 of [13]) is now easily proved. Indeed, if $A(u)=G^{(0)}(u) J\left(G^{(0)}\right)^{-1}$ holomorphically on $\mathbb{D}\left(u^{c}\right)$, where $J$ is Jordan, then $G^{(0)}$ satisfies $(10.1)$ on $\mathbb{D}\left(u^{0}\right)$ (and $J$ is constant). Since $G^{(0)}(u)$ is holomorphic on $\mathbb{D}\left(u^{c}\right)$, the $\omega_{j}$ must be as well, so that the vanishing conditions (2.22) must hold. Conversely, if $A$ is holomorphic on on $\mathbb{D}\left(u^{0}\right)$ and satisfies the vanishing conditions (2.22) (or, more weakly, if $d A=\sum_{j}\left[\omega_{j}, A\right] \mathrm{d} u_{j}$ on $\mathbb{D} \backslash \Delta$, which automatically implies (2.22)—see Remark 2.1), then $d G=\sum_{j} \omega_{j} \mathrm{~d} u_{j} G$ is integrable with holomorphic coefficients on $\mathbb{D}\left(u^{0}\right)$, and admits a fundamental matrix solution that can be chosen so that $\left(G^{(0)}\right)^{-1} A G^{(0)}(u)=J$ (the proof is as done before on $\mathbb{D}\left(u^{0}\right)$ ).

$\overline{22 \text { Up to the freedom } G \mapsto G G_{*}}$ where $G_{*}$ commutes with the Jordan form. 


\section{Appendix C. The normal form (8.3)}

We prove the expression (8.3) of Sect. 8, where it was sufficient to only consider the generic case of all $\lambda_{1}^{\prime}, \ldots, \lambda_{n}^{\prime} \notin \mathbb{Z}$ and no integer eigenvalues of $A$. A fundamental matrix solution in Levelt form at $\lambda=\lambda_{1}$ for system (8.1) has structure

$$
\stackrel{\circ}{\Psi}(\lambda)=G^{\left(p_{1}\right)}\left(I+\sum_{l=1}^{\infty} \mathfrak{G}_{l}\left(\lambda-\lambda_{1}\right)^{l}\right)\left(\lambda-\lambda_{1}\right)^{T^{\left(p_{1}\right)}}\left(\lambda-\lambda_{1}\right)^{R},
$$

with

$$
R=R_{1}+R_{2}+\cdots R_{\kappa}, \quad \kappa:=\max \left\{T_{i i}^{\left(p_{1}\right)}-T_{j j}^{\left(p_{1}\right)} \text { integer }\right\}
$$

where $R$ is a nilpotent matrix with $R_{i j} \neq 0$ only if $T_{i i}^{\left(\boldsymbol{p}_{1}\right)}-T_{j j}^{\left(\boldsymbol{p}_{1}\right)}$ is a positive integer. We prove that $R=0$ in our case. The formulae for $\left(\mathfrak{G}_{l}\right)_{i j}$ and $\left(R_{l}\right)_{i j}$ are obtained recursively by substituting the series into the differential system, and are as follows.

- If $T_{i i}^{\left(\boldsymbol{p}_{1}\right)}-T_{j j}^{\left(\boldsymbol{p}_{1}\right)}=l$ (positive integer), $\left(\mathfrak{G}_{l}\right)_{i j}$ is arbitrary, and

$$
\left(R_{l}\right)_{i j}=\left(\sum_{p=1}^{l-1}\left(\mathfrak{D}_{l-p} \mathfrak{G}_{l}-\mathfrak{G}_{l} R_{l-p}\right)+\mathfrak{D}_{l}\right)_{i j}
$$

- If $T_{i i}^{\left(\boldsymbol{p}_{1}\right)}-T_{j j}^{\left(\boldsymbol{p}_{1}\right)} \neq l$ (positive integer)

$$
\left(\mathfrak{G}_{l}\right)_{i j}=\frac{1}{T_{j j}^{\left(\boldsymbol{p}_{1}\right)}-T_{i i}^{\left(\boldsymbol{p}_{1}\right)}+l}\left(\sum_{p=1}^{l-1}\left(\mathfrak{D}_{l-p} \mathfrak{G}_{l}-\mathfrak{G}_{l} R_{l-p}\right)+\mathfrak{D}_{l}\right)_{i j}
$$

The claim that $R=0$ follows from two facts. First, if we evaluate at $u=u^{c}$ the isomonodromic fundamental matrix solution (6.24), we receive a fundamental matrix solution of (1.4) at $u=u^{c}$,

$$
\Psi^{\left(p_{1}\right)}\left(\lambda, u^{c}\right)=G^{\left(p_{1}\right)} \cdot U^{\left(p_{1}\right)}\left(\lambda, u^{c}\right) \cdot\left(\lambda-\lambda_{1}\right)^{T^{\left(p_{1}\right)}},
$$

which has $R=0$, because in the generic case here considered all $R^{(j)}=0$ in (6.24). The expression (11.2) belongs to the class of solutions (11.1).

The second fact is that other solutions in the class (11.1) may have different matrix exponents (see [27] and [13]; see also [14,20] for the case of Frobenius manifolds), but if $R$ corresponds to one solution, all the other solutions in the class can only have exponent

$$
\widetilde{R}=\mathcal{D}^{-1} R \mathcal{D}
$$

where $\mathcal{D}$ is an invertible matrix explained below. Now, since $R=0$ in (11.2), then by (11.3) all the other $\widetilde{R}=0$. This proves that (8.3) is the correct form. 
Finally, we explain (11.3). System (1.4) at $u=u^{c}$ is holomorphically equivalent to "Birkhoff-normal forms"

$$
\frac{\mathrm{d} \Psi}{\mathrm{d} \lambda}=\left(\frac{T^{\left(p_{1}\right)}}{\lambda-\lambda_{1}}+\sum_{l=1}^{\kappa} R_{l}\left(\lambda-\lambda_{1}\right)^{l}\right) \Psi \quad \text { and } \quad \frac{\mathrm{d} \widetilde{\Psi}}{\mathrm{d} \lambda}=\left(\frac{T^{\left(p_{1}\right)}}{\lambda-\lambda_{1}}+\sum_{l=1}^{\kappa} \widetilde{R}_{l}\left(\lambda-\lambda_{1}\right)^{l}\right) \widetilde{\Psi}
$$

which are related to each other by a gauge transformations $\Psi=\mathcal{D}(\lambda) \widetilde{\Psi}$, with $\mathcal{D}(\lambda)=$ $\mathcal{D}_{0}\left(I+\mathcal{D}_{0}\left(\lambda-\lambda_{1}\right)+\cdots+\mathcal{D}_{\kappa}\left(\lambda-\lambda_{1}\right)^{\kappa}\right)$, where $\operatorname{det}\left(\mathcal{D}_{0}\right) \neq 0$ and $\left[\mathcal{D}_{0}, T^{\left(p_{1}\right)}\right]=0$. Then, $\mathcal{D}:=\mathcal{D}_{0}\left(I+\mathcal{D}_{0}+\cdots+\mathcal{D}_{\kappa}\right)$.

Remark 11.1 In our case, the equations $R_{l}=0, l=1,2, \ldots, \kappa$ are conditions on the entries of $A\left(u^{c}\right)$. The above discussion shows that, in the isomonodromic case, such conditions turn out to be automatically satisfied with the only vanishing assumption $\left(A\left(u^{c}\right)\right)_{a b}=0$ for $u_{a}^{c}=u_{b}^{c}$. These conditions are equivalent to the conditions (4.24)(4.25) of Proposition 4.2 in [13], and probably more convenient. We will not enter into the tedious verification of the equivalence.

\section{References}

1. Balser, W., Jurkat, W.B., Lutz, D.A.: Birkhoff invariants and stokes' multipliers for meromorphic linear differential equations. J. Math. Anal. Appl. 71, 48-94 (1979)

2. Balser, W., Jurkat, W.B., Lutz, D.A.: A general theory of invariants for meromorphic differential equations. Part I, formal invariants. Funkcial. Evacioj 22, 197-221 (1979)

3. Balser, W., Jurkat, W.B., Lutz, D.A.: A general theory of invariants for meromorphic differential equations. Part II, proper invariants. Funkcial. Evacioj 22, 257-283 (1979)

4. Balser, W., Jurkat, W.B., Lutz, D.A.: On the reduction of connection problems for differential equations with irregular singular points to ones with only regular singularities. I. SIAM J. Math Anal. 12(5), 691721 (1981)

5. Birkhoff, G.D.: Singular points of ordinary linear differential equations. Trans. Am. Math. Soc. 10, 436-470 (1909)

6. Bolibruch, A.A.: The fundamental matrix of a Pfaffian system of Fuchs type. Izv. Akad. Nauk SSSR Ser. Mat. 41(5), 1084-1109, 1200 (1977)

7. Bolibruch, A.A.: On Isomonodromic deformations of Fuchsian systems. J. Dyn. Control Syst. 3, 589604 (1997)

8. Bolibruch, A.A.: On Isomonodromic confluence of Fuchsian singularities. Proc. Stek. Inst. Math. 221, 117-132 (1998)

9. Bridgeland, T., Toeldano Laredo, V.: Stokes factors and multilogarithms. J. reine und angew. Math. 682, 89-128 (2013)

10. Cotti, G.: Coalescence Phenomenon of Quantum Cohomology of Grassmannians and the Distribution of Prime Numbers. Int. Math. Res. Not. (2020) https://doi.org/10.1093/imrn/rnaa163. https://academic. oup.com/imrn/advance-article/doi/10.1093/imrn/rnaa163/5866263

11. Cotti, G.: Degenerate Riemann-Hilbert-Birkhoff problems, semisimplicity, and convergence of WDVV-potentials, (2020) arXiv:2011.04498

12. Cotti, G.: Riemann-Hilbert-Birkhoff inverse problem for semisimple flat F-manifolds, and convergence of oriented associativity potentials, (2021) arXiv:2105.06329

13. Cotti, G., Dubrovin, B., Guzzetti, D.: Isomonodromy Deformations at an Irregular Singularity with Coalescing Eigenvalues, Duke Math. J, (2019), arXiv:1706.04808 (2017)

14. Cotti, G., Dubrovin, B., Guzzetti, D.: Local Moduli of Semisimple Frobenius Coalescent Structures, SIGMA 16, 040, 105 p. (2020) https://www.emis.de/journals/SIGMA/2020/040/

15. Cotti, G., Dubrovin, B., Guzzetti, D.: Helix Structures in Quantum Cohomology of Fano Varieties, (2018) arXiv:1811.09235 
16. Cotti, G., Guzzetti, D.: Results on the extension of Isomonodromy deformations to the case of a resonant irregular singularity. Random Matrices Theory Appl. 7, 1840003 (2018). 27 pp

17. Cotti, G., Guzzetti, D.: Analytic Geometry of semisimple coalescent Frobenius Structures. Random Matrices Theory Appl. 6, 1740004 (2017). 36 pp

18. Doetsch, G.: Introduction to the Theory and Application of the Laplace Transformation. Springer, Berlin (1974)

19. Dubrovin, B.: Geometry of 2D topological field theories. Lect. Notes Math. 1620, 120-348 (1996)

20. Dubrovin, B.: Painlevé trascendents in two-dimensional topological field theory, In: "The Painlevé Property, One Century Later" edited by R. Springer, Conte (1999)

21. Dubrovin, B.: On Almost Duality for Frobenius Manifolds, Geometry, topology, and mathematical physics, 75-132. Am. Math. Soc. Transl. Ser. 2, 212 (2004)

22. Galkin, S., Golyshev, V., Iritani, H.: Gamma classes and quantum cohomology of Fano manifolds: gamma conjectures. Duke Math. J. 165(11), 2005-2077 (2016)

23. Guzzetti, D.: On Stokes matrices in terms of connection coefficients. Funkcial. Ekvac. 59(3), 383-433 (2016)

24. Guzzetti, D.: Deformations with a resonant irregular singularity, In: Proceedings of the Workshop "Formal and Analytic Solutions of Differential Equations", Alcalá, September 4-8, 2017. Springer (2018)

25. Guzzetti, D.: Notes on Non-Generic Isomonodromy Deformations, SIGMA 14, 087, 34 p (2018). https://www.emis.de/journals/SIGMA/2018/087/

26. Guzzetti, D.: Isomonodromy deformations with coalescing eigenvalues and applications, In: Filipuk, G., Lastra, A., Michalik, S., Takei, Y., Zoladek, H. (eds.) Complex Differential and Difference Equations, pp. 313-325. De Gruyter (2020)

27. Guzzetti, D.: Introduction to Linear ODEs in the Complex Domain and Isomonodromy Deformations - Lecture Notes for a Ph.D. Course at SISSA, Trieste

28. Haraoka, Y.: Linear Differential Equations in the Complex Domain, from classical theory to forefront, Suugaku Shobou, Tokyo (2015) (in Japanese), and Springer Lecture Notes in Mathematics 2271 (2020) (in English)

29. Hurtubise, J., Lambert, C., Rousseau, C.: Complete system of analytic invariants for unfolded differential linear systems with an irregular singularity of Poincaré rank k. Moscow Math. J. 14, 309-338 (2013)

30. Hsieh, P., Sibuya, Y.: Note on regular perturbations of linear ordinary differential equations at irregular singular points. Funkcial. Ekvac 8, 99-108 (1966)

31. Ince, E.L.: Ordinary Differential Equations. Dover, Illinois (1956)

32. Iwasaki, K., Kimura, H., Shimomura, S., Yoshida, M.: From Gauss to Painleve'. Aspect. Math. 16,(1991)

33. Jimbo, M., Miwa, T., Ueno, K.: Monodromy preserving deformations of linear ordinary differential equations with rational coefficients (I). Phys. D 2, 306 (1981)

34. Kedlaya, K.: Good formal structures for flat meromorphic connections, I: surfaces. Duke. Math. J. 154(2), 343-418 (2010)

35. Kedlaya, K.: Good formal structures for flat meromorphic connections, II: excellent schemes. J. Am. Math. Soc. 24, 183-229 (2011)

36. Klimes, M.: Confluence of Singularities of non-linear differential equations via Borel-Laplace transformations. J. Dyn. J. Dyn. Control Syst. 22, 285-324 (2016)

37. Klimes, M.: Analytic Classification of Families of Linear Differential Systems Unfolding a Resonant Irregular Singularity, SIGMA 16, 006, 46 p (2020). https://www.emis.de/journals/SIGMA/2020/006/

38. Klimes, M.: Confluence of singularities in hypergeometric systems. Funkcial. Ekvacioj 63, 183-197 (2020)

39. Loday-Richaud, M., Remy, P.: Resurgence, stokes phenomenon and alien derivatives for level-one linear differential systems. J. Differ. Equ. 250, 1591-1630 (2011)

40. Majima, H.: Asymptotic analysis for integrable connections with irregular singular points. Lecture Notes in Mathematics, vol. 1075. Springer-Verlag (1984)

41. Malgrange, B.: Sur les déformations isomonodromiques. II. Singularités irrégulières. Mathematics and physics (Paris, 1979/1982), 427-438, and Progress. Math., 37, Birkhäuser Boston, Boston, MA, 427-438 (1983)

42. Malgrange, B.: Deformations of differential systems. II. J. Ramanujan Math. Soc. 1, 3-15 (1986) 
43. Miwa, T.: Painlevé property of monodromy preserving deformation equations and the analyticity of $\tau$ functions. Publ. RIMS, Kyoto Univ. 17, 703-721 (1981)

44. Mazzocco, M.: Painlevé sixth equation as isomonodromic deformations equation of an irregular system. CRM Proc. Lecture Notes, Am. Math. Soc. 32, 219-238 (2002)

45. Mochizuki, T.: Good formal structure for meromorphic flat connections on smooth projective surfaces, in Algebraic analysis and around. Adv. Stud. Pure Math., Math. Soc. Japan 54, 223-253 (2009)

46. Mochizuki, T.: Wild harmonic bundles and wild pure twistor D-modules. Astérisque No. 340 x+607 pp. 5 (2011)

47. Mochizuki, T.: The Stokes structure of a good meromorphic flat bundle. J. Inst. Math. Jussieu 10(3), 675-712 (2011)

48. Mochizuki, T.: Holonomic D-modules with Betti structure. Mém. Soc. Math. Fr. (N.S.) No. 138-139, viii+205 pp (2014)

49. Remy, P.: Matrices de Stokes-Ramis et constantes de connexion pour les systèmes différentiels linéaires de niveau unique. Ann. Fac. Sci. Toulouse Math. (6) 21(1), 93-150 (2012)

50. Sabbah, C.: Équations différentielles à points singuliers irréguliers en dimension 2. Ann. Isnt. Fourier 43, 1619-1688 (1993)

51. Sabbah, C.: Équations différentielles à points singuliers irréguliers et phénomène de Stokes en dimension 2, Astérisque, vol 263, Société Matématique de France, (2000)

52. Sabbah, C.: Integrable deformations and degenerations of some irregular singularities, Publ. RIMS Kyoto Univ. 57, n. 3-4, (2021) to appear. arXiv:1804.00426 (2017)

53. Sabbah, C.: A short proof of a theorem of Cotti, Dubrovin and Guzzetti, (2021) arXiv:2103.16878

54. Sibuya, Y.: Simplification of a system of linear ordinary differential equations about a singular point. Funkcial. Ekvac 4, 29-56 (1962)

55. Sibuya, Y.: Perturbation of linear ordinary differential equations at irregular singular points. Funkcial. Ekvac 11, 235-246 (1968)

56. Schäfke, R.: The connection problem for two neighboring regular singular points of general complex ordinary differential equations. SIAM J. Math. Anal. 11, 863-875 (1980)

57. Schäfke, R.: A connection problem for a regular and an irregular singular point of complex ordinary differential equations. SIAM J. Math. Anal. 15, 253-271 (1984)

58. Schäfke, R.: Über das globale analytische Verhalten der Normallosungen von $(s-B) v^{\prime}(s)=(B+$ $\left.t^{-1} A\right) v(s)$ und zweier Arten von assoziierten Funktionen. Math. Nachr. 121, 123-145 (1985)

59. Schäfke, R.: Confluence of several regular singular points into an irregular singular one. J. Dyn. Control Syst. 4, 401-424 (1998)

60. Teyssier, J.B.: Moduli of Stokes torsors and singularities of differential equations, (2018). arXiv: 1802.00289

61. Ugaglia, M.: On a Poisson structure on the space of stokes matrices. Int. Math. Res. Notices 1999(9), 473-493 (1999)

62. Wasow, W.: Asymptotic Expansions for Ordinary Differential Equations. Dover, Illinois (1965)

63. Yoshida, M., Takano, K.: On a linear system of Pfaffian equations with regular singular points. Funkcial. Ekvac. 19(2), 175-189 (1976)

Publisher's Note Springer Nature remains neutral with regard to jurisdictional claims in published maps and institutional affiliations. 